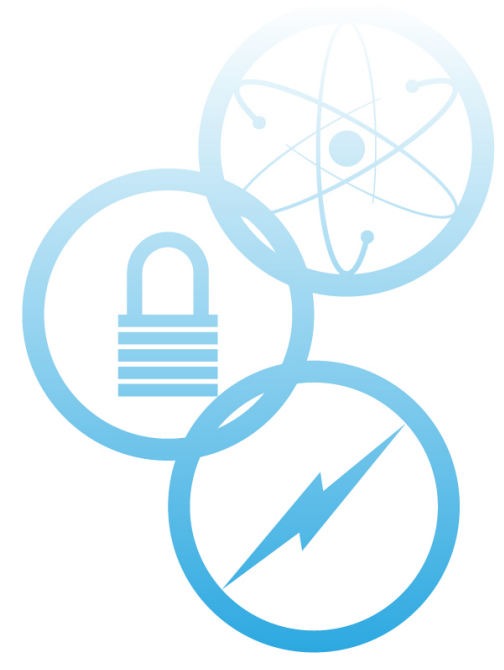

\title{
Summary of BISON Milestones: NEAMS FY-20 Report
}

\section{September 2020}

Idaho National Laboratory

A. Casagranda

L. K. Aagesen

J.-H. Ke

W. Jiang

J. D. Hales

A. Toptan

K. A. Gamble

$X .-Y$. Liu

S. R. Novascone

Los Alamos National Laboratory

C. Matthews

Southwestern Scientific

D. S. Stafford

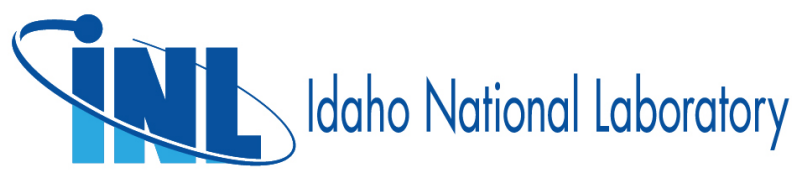




\section{DISCLAIMER}

This information was prepared as an account of work sponsored by an agency of the U.S. Government. Neither the U.S. Government nor any agency thereof, nor any of their employees, makes any warranty, expressed or implied, or assumes any legal liability or responsibility for the accuracy, completeness, or usefulness, of any information, apparatus, product, or process disclosed, or represents that its use would not infringe privately owned rights. References herein to any specific commercial product, process, or service by trade name, trade mark, manufacturer, or otherwise, does not necessarily constitute or imply its endorsement, recommendation, or favoring by the U.S. Government or any agency thereof. The views and opinions of authors expressed herein do not necessarily state or reflect those of the U.S. Government or any agency thereof. 


\title{
Summary of BISON Milestones: NEAMS FY-20 Report
}

\author{
Idaho National Laboratory \\ A. Casagranda \\ L. K. Aagesen \\ J.-H. Ke \\ W. Jiang \\ J. D. Hales \\ A. Toptan \\ K. A. Gamble \\ X.-Y. Liu \\ S. R. Novascone
}

Los Alamos National Laboratory

C. Matthews

Southwestern Scientific

D. S. Stafford

September 2020

\begin{abstract}
Idaho National Laboratory
Computational Mechanics and Materials

Idaho Falls, Idaho 83415
\end{abstract}

\author{
Prepared for the \\ U.S. Department of Energy \\ Office of Nuclear Energy \\ Under DOE Idaho Operations Office \\ Contract DE-AC07-05ID14517
}




\section{Contents}

1 INTRODUCTION 6

2 MILESTONE SUMMARY AND COMPLETION SCHEDULE $\quad 7$

3 LLS-INFORMED SWELLING MODEL FOR METALLIC FUEL

A. Casagranda

L. K. Aagesen

J.-H. Ke

S. Novascone

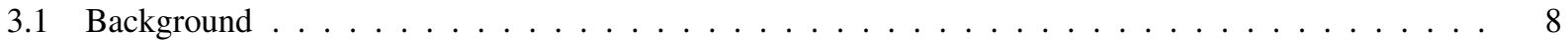

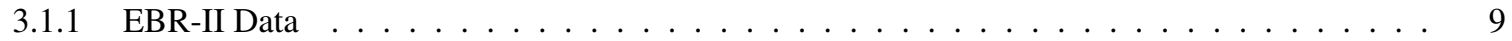

3.2 Dakota Parameter Sensitivity and Optimization Processes . . . . . . . . . . . . . . . 9

3.3 Fission Gas Release Optimization . . . . . . . . . . . . . . . . . . . . . . 9

3.3.1 Volumetric Swelling Behavior . . . . . . . . . . . . . . . . . . . . 16

3.4 Lower-Length-Scale-Informed Modeling . . . . . . . . . . . . . . . . . . . . . . . . 18

3.5 Bubble Number Density . . . . . . . . . . . . . . . . . . . . . . . . . . . . . . . . . . . . 21

3.6 Anisotropic Swelling Optimization . . . . . . . . . . . . . . . . . . . . . . 23

3.7 Discussion . . . . . . . . . . . . . . . . . . . . . . 26

3.8 Conclusions . . . . . . . . . . . . . . . . . . . . . . . . 26

3.9 Future Work . . . . . . . . . . . . . . . . . . . . . . . . 26

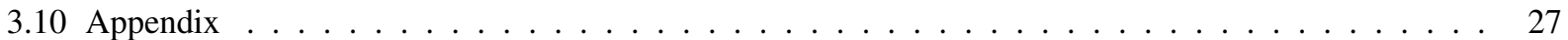

3.10 .1 Input Files . . . . . . . . . . . . . . . . . . . . . . . . . . . . . . 27

3.10 .2 Running Process . . . . . . . . . . . . . . . . . . . . . . . . . . 27

3.10 .3 Dakota Input File . . . . . . . . . . . . . . . . . . . . . . . . . . . . . . . . . . . . . . . . . . . . . . . . . . . .

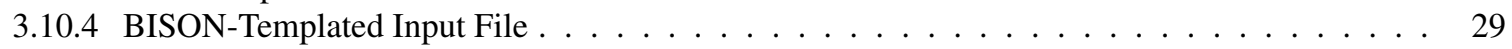

3.10 .5 Optimization Run Shell Script . . . . . . . . . . . . . . . . . . . . . . . . . . . . . 29

3.10 .6 Python Objective Function Script . . . . . . . . . . . . . . . . . . . . . . 30

3.10 .7 Updated Anisotropic Swelling Calibration . . . . . . . . . . . . . . . . . . . . . . . . . 31

4 DEMONSTRATE BISON CAPABILITY FOR HTGR FUEL APPLICATIONS

W. Jiang

J.D. Hales

A. Toptan

K.A. Gamble

S.R. Novascone

X.-Y. Liu

C. Matthews

4.1 TRISO UCO Material Models . . . . . . . . . . . . . . . . . . . . . . . . 36

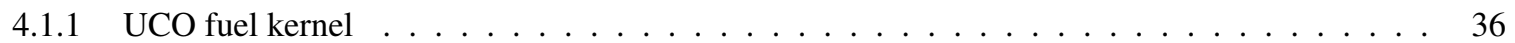

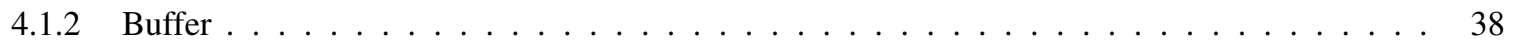

$4.1 .3 \mathrm{PyC} \ldots \ldots \ldots \ldots \ldots \ldots$

$4.1 .4 \mathrm{SiC} \ldots \ldots \ldots \ldots \ldots \ldots \ldots \ldots$

4.2 Fission gas release model . . . . . . . . . . . . . . . . . . . . . . . 44 
4.2 .1 Fission gas ... . . . . . . . . . . . . . . . . . . . . . . 44

4.2.2 Diffussion coefficients calculated by lower length scale modeling . . . . . . . . . . . . . 46

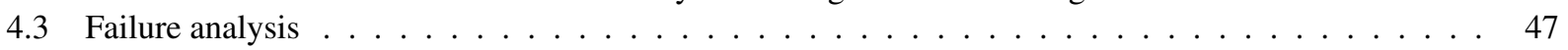

4.3 .1 Failure modes . . . . . . . . . . . . . . . . . . . . . . . . . . . . . . . . . . . . . . . . . .

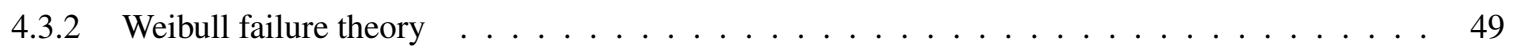

4.3 .3 High-fidelity analysis . . . . . . . . . . . . . . . . . . . . . 50

4.3.4 Monte Carlo Scheme . . . . . . . . . . . . . . . . . . . . . . 51

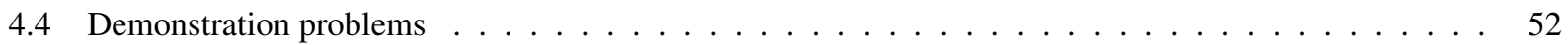

4.4.1 Incorporate diffusion coefficients from LANL . . . . . . . . . . . . . . . . . . . . . 52

4.4.2 Benchmark BISON against PARFUME . . . . . . . . . . . . . . . . . . . . . . . . . .

4.4.3 AGR-5/6/7 Irradiation Test Predictions . . . . . . . . . . . . . . . . . . . 56

4.5 Conclusions . . . . . . . . . . . . . . . . . . . . . . . . . . 61

4.6 Future Work . . . . . . . . . . . . . . . . . . . . . . 61

5 DEVELOP AND DEMONSTRATE INITIAL CAPABILITY TO SIMULATE UN/UC FUEL

\section{S. Stafford}

C. Matthews

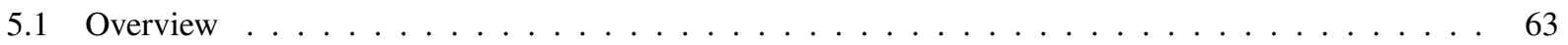

5.2 Mixed Carbides . . . . . . . . . . . . . . . . . . . . . . . . . 64

5.2 .1 Thermal Properties . . . . . . . . . . . . . . . . . . . . . 64

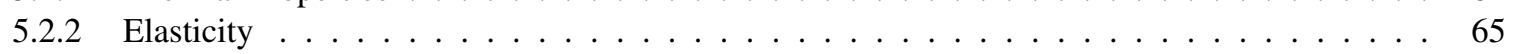

5.2 .3 Thermal Expansion . . . . . . . . . . . . . . . . . . . . . . . 65

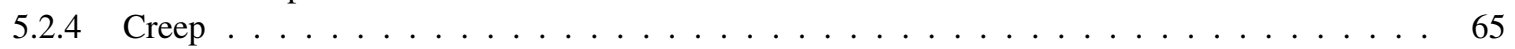

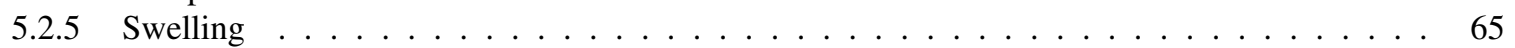

5.3 Mixed Nitrides . . . . . . . . . . . . . . . . . . . . . . . 66

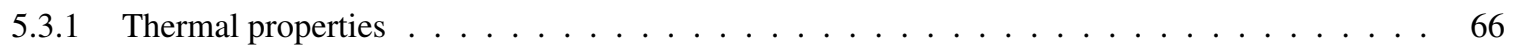

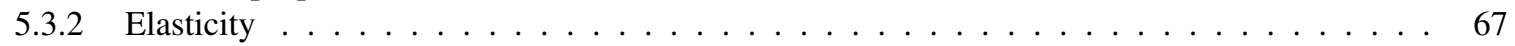

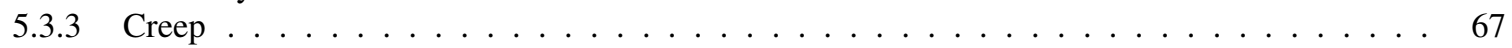

5.3 .4 Thermal Expansion . . . . . . . . . . . . . . . . . . . . . . 67

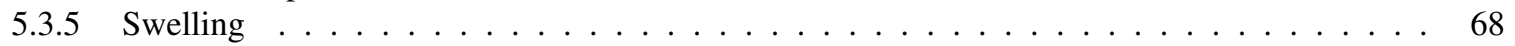

5.4 Fuel Rod Demonstrations . . . . . . . . . . . . . . . . . . . . . . 68

5.4.1 Carbides: EBR-II WSA-32 Experiment . . . . . . . . . . . . . . . . . . 68

5.4 Nitrides: EBR-II K-4 Experiment . . . . . . . . . . . . . . . . . . . . . 70

6 ACTIVITIES

6.1 User Training and Support . . . . . . . . . . . . . . . . . . . . . 73

6.2 Particle Fuel Model Assessment . . . . . . . . . . . . . . . . . . . . . . . 73

7 FUTURE WORK

8 ACKNOWLEDGEMENTS 


\section{Chapter 1}

\section{INTRODUCTION}

This summary report contains an overview of work performed under the work package entitled "FY 2020 NEAMS Advanced Fuels Performance," which is focused on the development and support of the fuel performance code BISON [1]. The second chapter lists FY-20 milestones titles, completion schedule, and milestone level. Subsequent chapters summarize and demonstrate completion of the milestones. The last chapter outlines FY-21 proposed future work.

In FY-20, the NEAMS program emphasized development of BISON for its application to advanced reactors. While there are a variety of advanced fuel concepts, based on interaction with industry and the Nuclear Regulatory Commission, the fuel types we chose to develop were metallic fast reactor, UN/UC and particle fuels.

The last chapter of this report documents proposed work for FY-21. We plan to continue work on metallic and particle fuel in terms of developing/calibrating models and to begin rigorous validation/assessment for both fuel types. Due to the merger of the NEAMS and CASL programs, FY-21 will see a return to light water reactor model development and simulation; this time focused on advanced technology fuels. Additionally, we seek to fundamentally improve BISON. As such, we plan improvements to BISON and MOOSE in terms of algorithmic robustness, performance, ease-of-use, and quality assurance. See a detailed write-up in section 7.

\section{Bibliography}

[1] R. L. Williamson, J. D. Hales, S. R. Novascone, M. R. Tonks, D. R. Gaston, C. J. Permann, D. Andrs, and R. C. Martineau. Multidimensional multiphysics simulation of nuclear fuel behavior. Journal of Nuclear Materials, 423:149-163, 2012. 


\section{Chapter 2}

\section{MILESTONE SUMMARY AND COMPLETION SCHEDULE}

FY 2020 Milestones and the completion dates are listed in Table 2.1. The milestones are listed by topic (metallic fuel, particle fuel, and UN/UC). The following chapters contain a short description of each milestone and references to related detailed documentation. Where applicable, a representative technical result from the work is included.

Table 2.1: FY 2020 Milestones for NEAMS INL-engineering scale fuel performance effort

\begin{tabular}{lcc}
\hline Milestone & Completion Date & MS Level \\
\hline $\begin{array}{l}\text { Demonstrate a physics-based, lower-length-scale-informed } \\
\text { swelling model that predicts EBR-II observations }\end{array}$ & $6 / 12 / 20$ & M2 \\
\hline Demonstrate BISON capability for HTGR fuel applications & $9 / 30 / 20$ & M3 \\
\hline $\begin{array}{l}\text { Develop and demonstrate initial capability to simulate UN/UC } \\
\text { fuel }\end{array}$ & $9 / 30 / 20$ & M3 \\
\hline
\end{tabular}

This report documents the efforts performed in FY 2020 for the milestones listed in the table above and constitutes the completion of them. 


\section{Chapter 3}

\section{LLS-INFORMED SWELLING MODEL FOR METALLIC FUEL}

A. Casagranda

L. K. Aagesen

J.-H. Ke

S. Novascone

The purpose of this report is to document improvements in BISON for predicting the swelling and fission gas release (FGR) behavior of metallic fuel. Building upon previous work, we utilized historical EBR-II experimental data to calibrate the FGR and swelling-related parameters in the code. In addition, we leveraged the activities of the lowerlength-scale team within this project to inform the porosity interconnection parameters in BISON. The details of these efforts are described in the following sections.

This document also constitutes the completion of the NEAMS milestone titled "M2MS-20IN0201021 - Demonstrate a physics-based, lower-length-scale-informed swelling model that predicts EBR-II observations." The description of that milestone is: "It is important to accurately predict swelling and corresponding porosity interconnection (fission gas release). By applying lessons learned from prior years of model development and our increasing familiarity with EBR-II data, we plan to calibrate parameters in current and emerging lower length scale informed engineering scale models and demonstrate this in a small subset of assessment cases. Furthermore, we plan to leverage lowerlength scale knowledge and models such that we have a strong, physics-based model calibration. The data we plan to use is from EBR-II, using both the IMIS and FIPD (ANL) databases. We also plan to use new data from AFC experiments that are planned for FY20. The input we plan to receive from lower length scale work at INL is a model that accounts for bubble number density (rather than assuming a constant value) which is based on microstructure evolution. From LANL, we expect to incorporate their work on consistent viscoplastic models with application to complex, phase-specific properties of metallic fuel."

\subsection{Background}

The default parameters for many of the metallic fuel material models in BISON were set using values from the original formulation. Use of these parameters led to simulation results that did not compare favorably to post-irradiation examination (PIE) results (Figure 3.1). Therefore, an effort was made to calibrate the BISON material models to the EBR-II data using the optimization capabilities of the Dakota software. The first set of models to be calibrated was the FGR model in the UPuZr family of material models.

It is important to put model parameter calibration into context. This report demonstrates and optimizes the initial capabilities of existing models. Additional comparisons to data and calibration efforts will be performed as new models are developed. The methods outlined in this report are fairly generic and can be applied to any model parameter and experimental dataset as long as an objective function can be computed and used within the Dakota optimization process. 


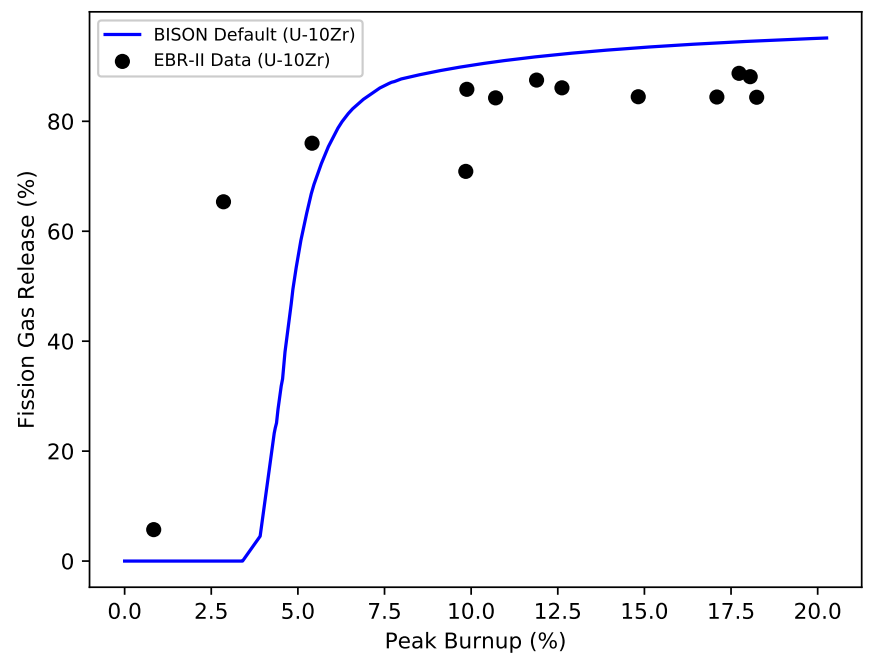

Figure 3.1: Comparison of BISON default FGR predictions and U-10Zr metallic fuel EBR-II experimental data.

\subsubsection{EBR-II Data}

A figure from an EBR-II publication [1] was used to extract FGR data for three different fuel compositions (U-10Zr, $\mathrm{U}-8 \mathrm{Pu}-10 \mathrm{Zr}$, and $\mathrm{U}-19 \mathrm{Pu}-10 \mathrm{Zr})$. The experimental data points were extracted from these plots and used to create CSV files for use in both plotting and the calibration process.

In addition to EBR-II experimental data published in the open literature, the Fuels Irradiation and Physics Database (FIPD) is available for providing fission gas release data as well as other PIE information for EBR-II experiments and individual pins [2].

\subsection{Dakota Parameter Sensitivity and Optimization Processes}

The Dakota software has numerous capabilities for parameter sensitivity studies, as well as in terms of optimization processes [3]. Both of these features have been used to analyze BISON simulations and calibrate material model parameters. The optimization methods will be discussed in this section in more detail.

Dakota supports multiple optimization approaches such as gradient-based, derivative-free local, and global methods applicable to various engineering problems. The software also provides hybrid approaches for combining methods to improve the robustness of the optimization process.

When using Dakota, we found that the hybrid approach of using a pattern search followed by a Quasi-Newton method met our requirements. This combined approach was robust and converged in all cases. A more detailed discussion of optimization techniques and the advantages/disadvantages of each is available in the Dakota User's Manual [3].

\subsection{Fission Gas Release Optimization}

The process used to optimize the FGR parameters in the BISON metallic fuel models utilizes the available EBR-II experimental data. The published data (shown in Figure 3.2) includes three compositions: $\mathrm{U}-10 \mathrm{Zr}, \mathrm{U}-8 \mathrm{Pu}-10 \mathrm{Zr}$, and $\mathrm{U}-19 \mathrm{Pu}-10 \mathrm{Zr}$. Two of the compositions ( $\mathrm{U}-10 \mathrm{Zr}$ and $\mathrm{U}-19 \mathrm{Pu}-10 \mathrm{Zr}$ ) will be used to actually optimize the FGR parameters and provide a "fit" for the plutonium content in the fuel. The remaining composition (U-8Pu-10Zr) can then be used to determine how well the optimized parameters model the FGR behavior. 


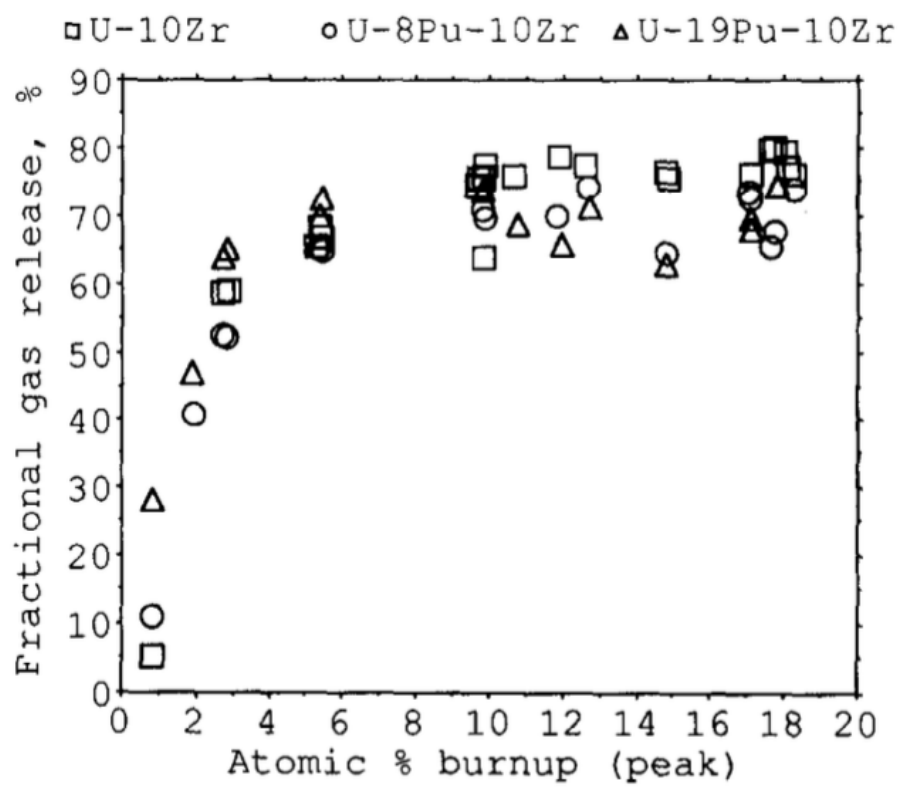

Figure 3.2: EBR-II fission gas release experimental data [1].

The BISON model used in the optimization process is based on the X441 assessment case in the BISON code repository. However, several changes were made to the model in order to speed up the run time and enable the Dakota optimization process to complete in a reasonable amount of time. First, the mesh was coarsened - especially in the axial direction (40 elements instead of 260) — but also in the radial direction for both the fuel and cladding. The linear power is the same as in the assessment case, but the end time of the simulation was extended to generate more burnup in the fuel and cause the comparison of the BISON results to the EBR-II experimental data to overlap fully. Finally, the model uses the standard metallic fuel output capability currently in development within BISON. This feature is being evaluated for inclusion in the code.

Table 3.1: Fission gas release model parameters.

\begin{tabular}{|c|c|}
\hline Parameter & Default Value \\
\hline Bubble Number Density & $1 \mathrm{e} 20$ \\
Interconnection Initiating Porosity & 0.23 \\
Interconnection Terminating Porosity & 0.25 \\
Critical Porosity & 0.24 \\
Fractional FGR Pre-interconnection & 0.8 \\
Fractional FGR Post-interconnection & 1.0 \\
\hline
\end{tabular}

Using the steps outlined in the appendix, the Dakota optimization process was applied to the U-10Zr experimental data, starting with the original default FGR-related model parameters. These values are summarized in Table 3.1.

For context, a brief description of the swelling and FGR process for zirconium-based metallic fuel is provided here. As the fuel fissions, gases and pores form within the fuel. The gases diffuse through the fuel and collect in the pores, and rapid swelling occurs. The pores eventually coalesce, and when the porosity reaches a value of about $24 \%$, corresponding to an experimentally observed swelling of about 33\%, the voids form an interconnected path through the fuel, fission gas is released, and swelling dramatically slows. The values of the parameters in Table 3.1 are based on these observations. To ensure a numerically smooth transition between porosity interconnection and termination, $+/-1 \%$ is added to the critical porosity value (24\%), and a smoothing function is used to slowly initiate FGR and decrease gaseous swelling. The fractional fission gas released pre- and post-interconnection represents the amount of FGR when the pores begin to interconnect ( $80 \%$ FGR at 23\% porosity) and once interconnection is complete (100\% 
FGR after $25 \%$ porosity is reached). The values of these parameters are similar to the outcomes determined from the bubble interconnection results from lower-length-scale calculations in Section 3.4. The bubble number density is also included in the calibration, due to its impact on swelling.

Figure 3.3 shows the FGR results using the default values (i.e., the starting point for the optimization process) and optimized FGR behavior, as well as the parameters for each calculation. The process was repeated using a different value for the bubble number density, $\mathrm{N}$, to determine the effect of the starting point for the optimization process on the results of the Dakota optimization. This was suggested by the lower-length-scale team and resulted in exposing a dependence of the results on the starting values for the optimization. Figure 3.4 shows the results from this run, and that the final optimized parameters are not significantly changed by the starting value of N. Figure 3.5 combines the optimized results, graphically illustrating the similarity of the Dakota solutions.

However, a similar set of plots for the composition U-19Pu-10Zr (Figures 3.6 to 3.8) shows that the starting value of the bubble number density can significantly influence the final optimized results, especially at the higher range of peak burnup. The optimization process applied to the $\mathrm{U}-8 \mathrm{Pu}-10 \mathrm{Zr}$ composition also shows that results depend on the initial bubble number density (Figures 3.9 to 3.11), but the main difference in this case seems to be at the low range of peak burnup. In addition, the FGR parameters that most influence the initiation of FGR (i.e., bubble number density and initial fractional FGR) show the largest difference, due to the starting value of N (Figure 3.11). This is believed to be due to the data points at the low end of peak burnup having a significant effect on the optimization process. The data point at approximately $1 \%$ is the "anchoring" point for the optimization with a starting value of $1 \mathrm{e} 16$ for N; however, with a starting value of $1 \mathrm{e} 20$ for $\mathrm{N}$, the "anchoring" point is the data point at $2.5 \%$ peak burnup.

Finally, the optimized parameters for the $\mathrm{U}-10 \mathrm{Zr}$ and $\mathrm{U}-19 \mathrm{Pu}-10 \mathrm{Zr}$ fuel compositions were used to develop a correlation between the parameter values and the plutonium content in the fuel. This correlation was then used to compute parameter values for the $\mathrm{U}-8 \mathrm{Pu}-10 \mathrm{Zr}$ fuel composition, and a BISON model was run using those values. This approach uses EBR-II data regarding the extreme values of plutonium content in the optimization process, while reserving the $\mathrm{U}-8 \mathrm{Pu}-10 \mathrm{Zr}$ data to check the correlation. Figure 3.12 shows a comparison of the BISON prediction based on the interpolated values and the optimization process applied directly to the $\mathrm{U}-8 \mathrm{Pu}-10 \mathrm{Zr}$ data. The interpolated values provide a relatively good prediction of $\mathrm{U}-8 \mathrm{Pu}-10 \mathrm{Zr}$ FGR behavior, especially considering the scatter in the data at higher burnup. Therefore, a correlation for the model parameters using this method is a reasonable means of approximating FGR behavior for fuel compositions between zero and 19\% plutonium.

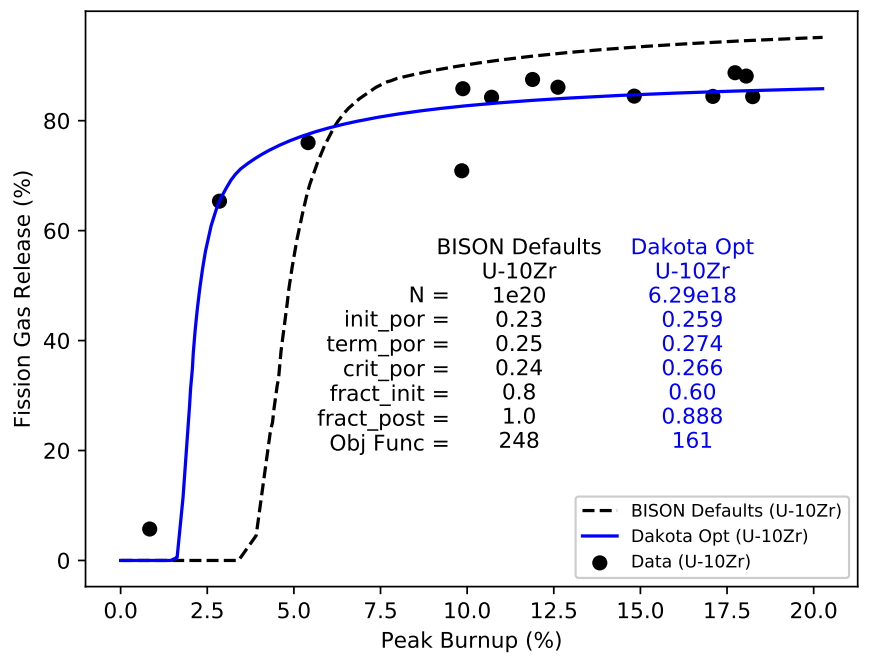

Figure 3.3: Comparison of FGR predictions using BISON default values as an initial guess for Dakota optimization and optimized parameters for U-10Zr EBR-II metallic fuel. 


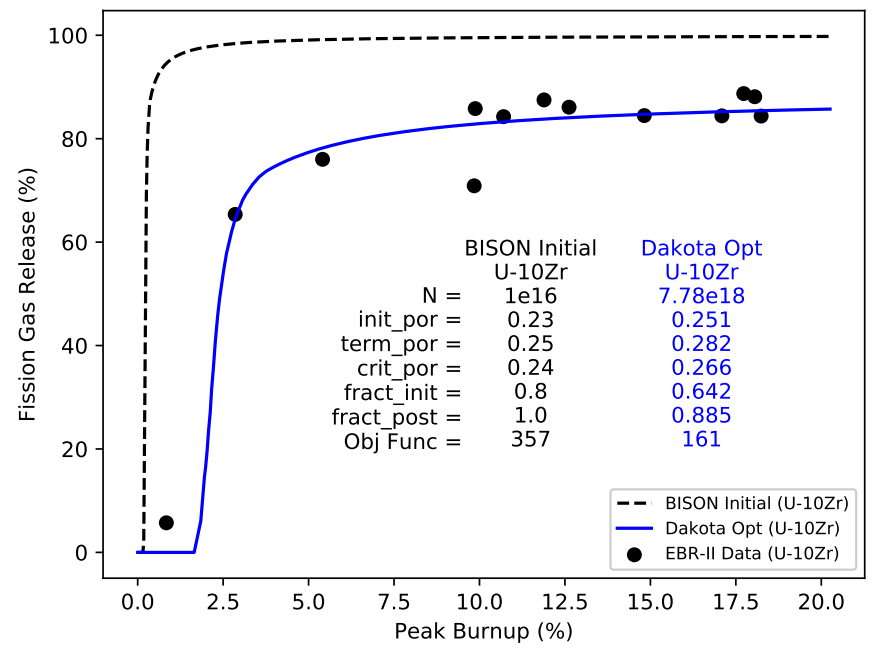

Figure 3.4: Comparison of FGR predictions using BISON defaults with $\mathrm{N}=1 \mathrm{E} 16$ as an initial guess for Dakota optimization and optimized results for U-10Zr EBR-II metallic fuel.

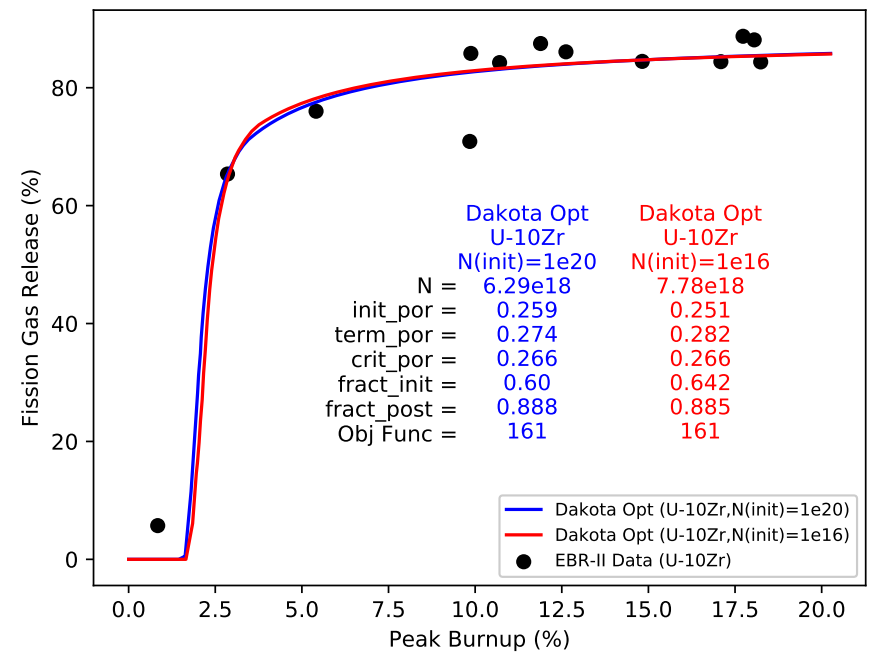

Figure 3.5: Comparison of optimized Dakota results using $\mathrm{N}=1 \mathrm{E} 20$ and $\mathrm{N}=1 \mathrm{E} 16$ as initial guesses in BISON simulations for U-10Zr EBR-II metallic fuel. 


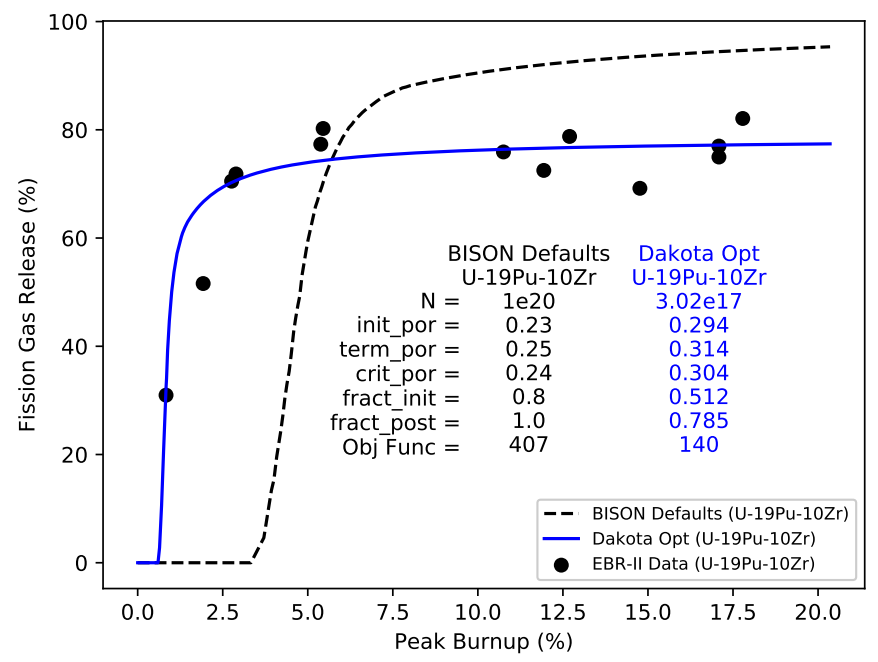

Figure 3.6: Comparison of FGR predictions using BISON default values as an initial guess for Dakota optimization and optimized parameters for U-19Pu-10Zr EBR-II metallic fuel.

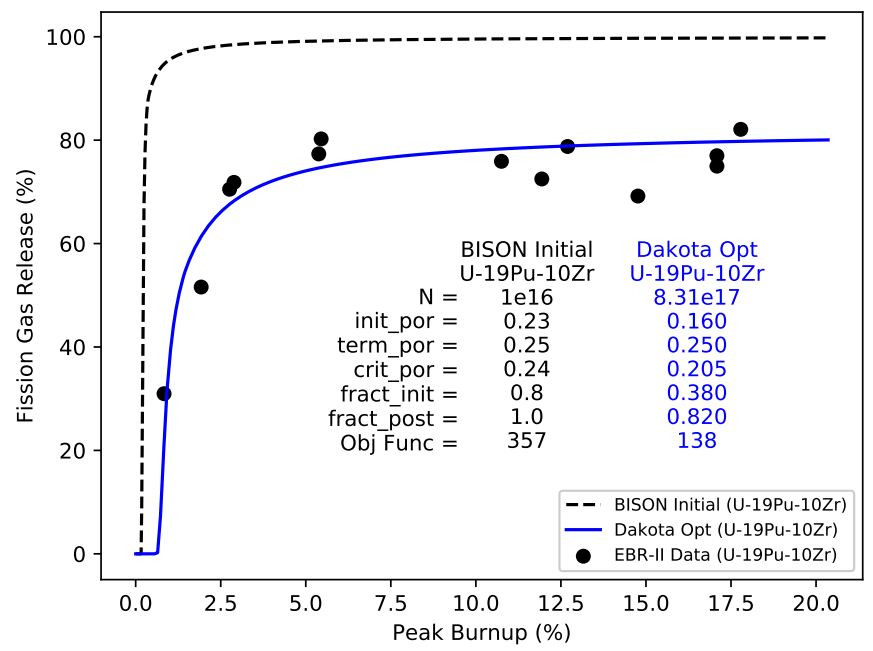

Figure 3.7: Comparison of FGR predictions using BISON defaults with $\mathrm{N}=1 \mathrm{E} 16$ as an initial guess for Dakota optimization and optimized results for $\mathrm{U}-19 \mathrm{Pu}-10 \mathrm{Zr}$ EBR-II metallic fuel. 


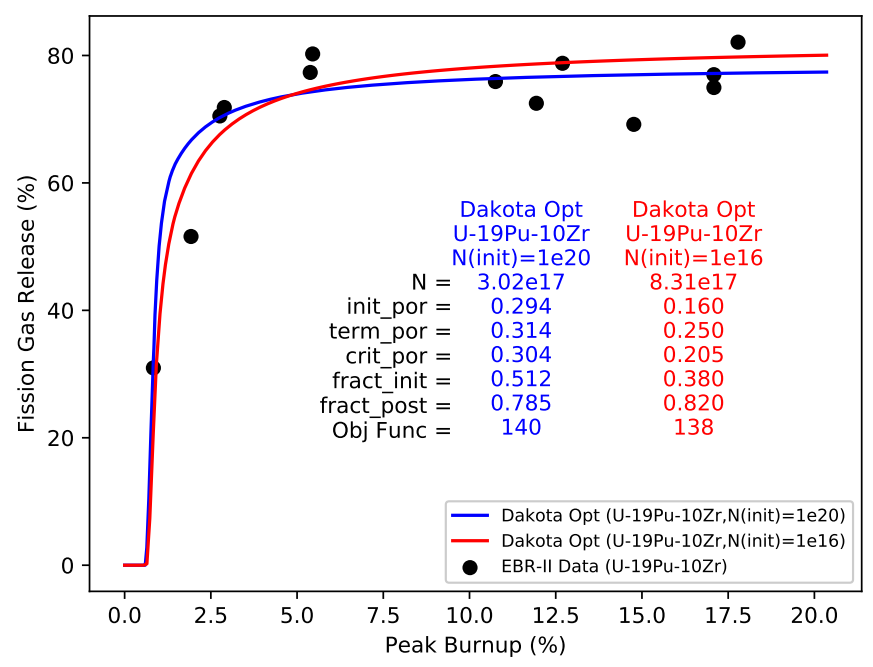

Figure 3.8: Comparison of optimized Dakota results using $\mathrm{N}=1 \mathrm{E} 20$ and $\mathrm{N}=1 \mathrm{E} 16$ as initial guesses in BISON simulations for U-19Pu-10Zr EBR-II metallic fuel.

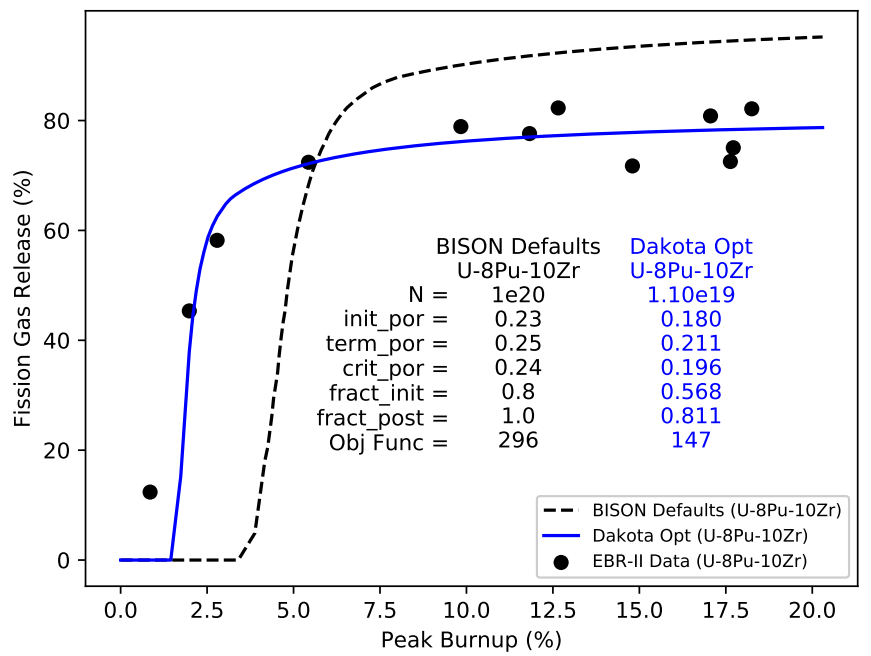

Figure 3.9: Comparison of FGR predictions using BISON default values as an initial guess for Dakota optimization and optimized parameters for $\mathrm{U}-8 \mathrm{Pu}-10 \mathrm{Zr}$ EBR-II metallic fuel. 


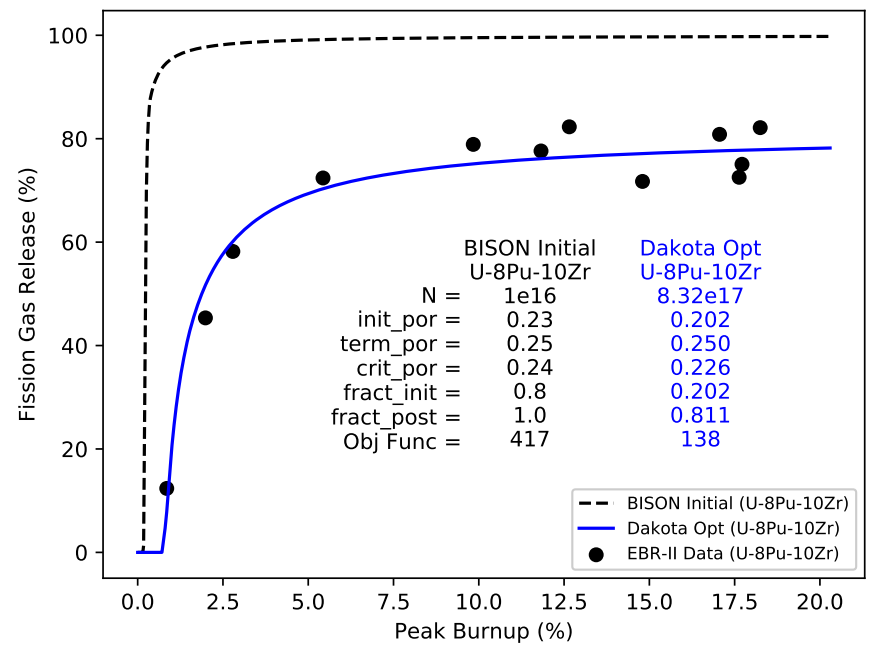

Figure 3.10: Comparison of FGR predictions using BISON defaults with $\mathrm{N}=1 \mathrm{E} 16$ as an initial guess for Dakota optimization and optimized results for U-8Pu-10Zr EBR-II metallic fuel.

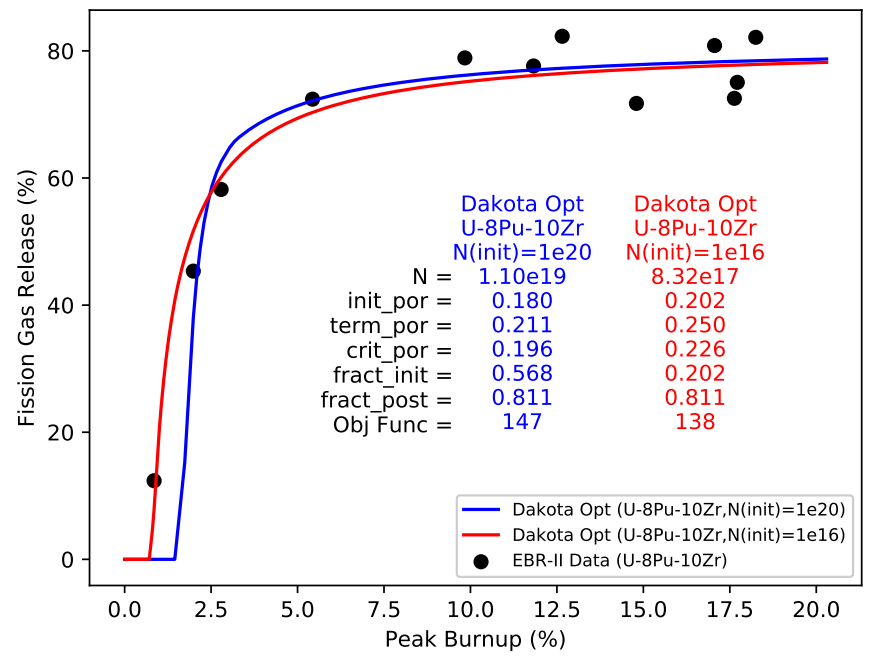

Figure 3.11: Comparison of optimized Dakota results using $\mathrm{N}=1 \mathrm{E} 20$ and $\mathrm{N}=1 \mathrm{E} 16$ as initial guesses in BISON simulations for U-8Pu-10Zr EBR-II metallic fuel. 


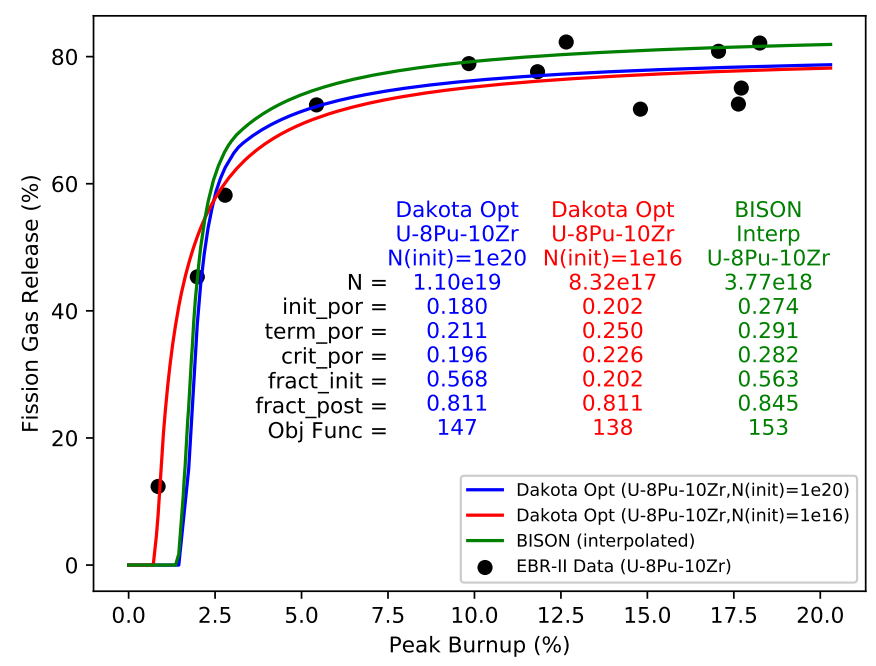

Figure 3.12: Comparison of optimized Dakota results for $\mathrm{U}-8 \mathrm{Pu}-10 \mathrm{Zr}$ and interpolated values for the FGR parameters using $\mathrm{U}-10 \mathrm{Zr}$ and $\mathrm{U} 1-9 \mathrm{Pu}-10 \mathrm{Zr}$ as bounding cases.

\subsubsection{Volumetric Swelling Behavior}

Calibration of the FGR parameters for metallic fuel also influences swelling behavior. Plotting the volumetric strain as a function of peak burnup for the default and optimized results from the above-described calibration runs illustrates a few trends. In particular, Figures 3.13 to 3.15 show that the initial slope of the optimized curves increases as the bubble number density decreases. This is due to the increased swelling rate and faster FGR for lower values of N. Also, the initial magnitude of the volumetric strain scales with the value of the initiating interconnection porosity. As the initiating interconnection porosity increases, more swelling is required before interconnection occurs and FGR can happen. Therefore, the volumetric strain curves for $\mathrm{U}-10 \mathrm{Zr}$ and $\mathrm{U}-19 \mathrm{Pu}-10 \mathrm{Zr}$ are higher than the default results; however, the volumetric strain curve for $\mathrm{U}-8 \mathrm{Pu}-10 \mathrm{Zr}$ is lower than the default. Thus, there is a direct correlation between FGR calibration and the swelling behavior of the fuel. 


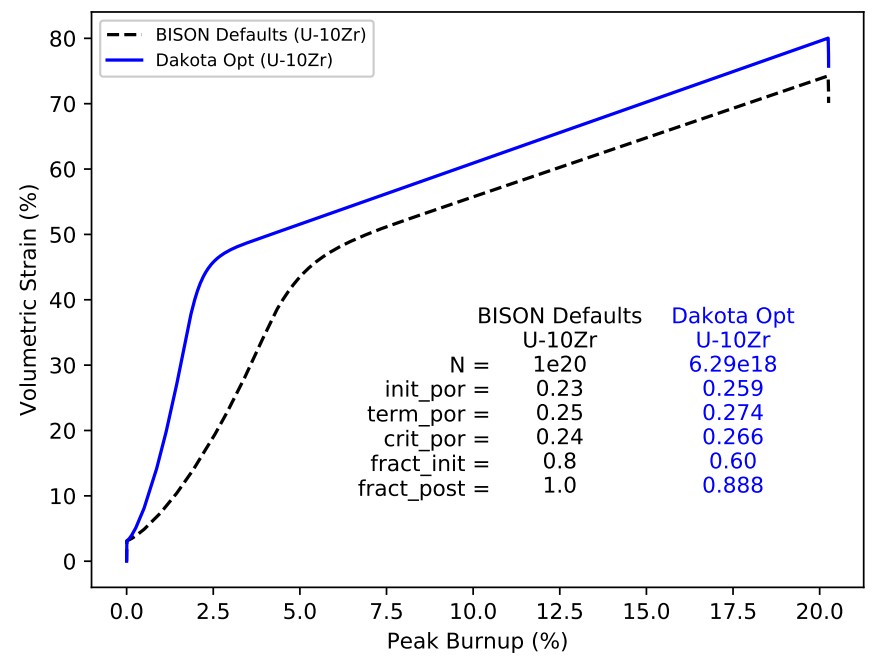

Figure 3.13: Comparison of volumetric strain versus burnup for the default values of the FGR parameters and the Dakota-optimized values for U-10Zr.

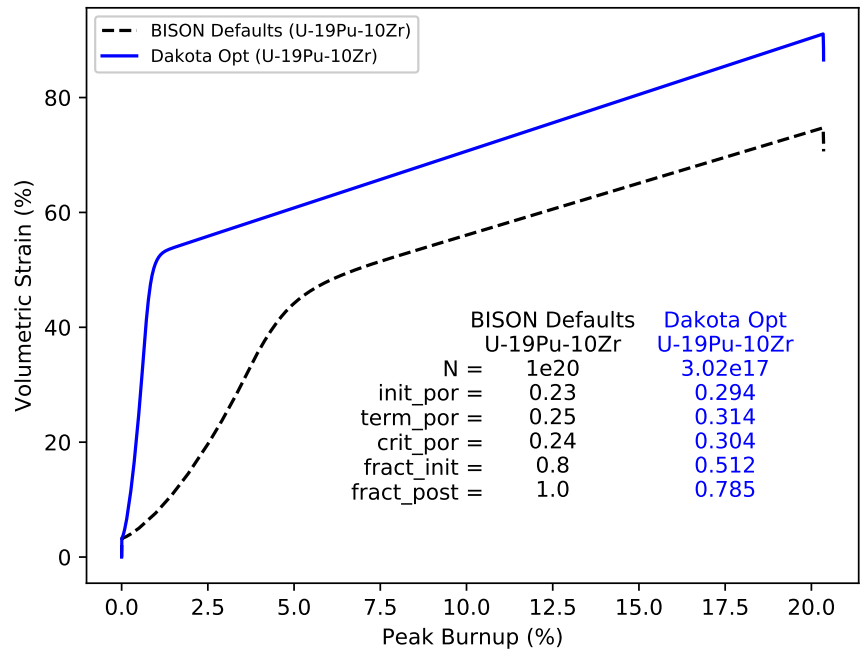

Figure 3.14: Comparison of volumetric strain versus burnup for the default values of the FGR parameters and the Dakota-optimized values for $\mathrm{U}-19 \mathrm{Pu}-10 \mathrm{Zr}$. 


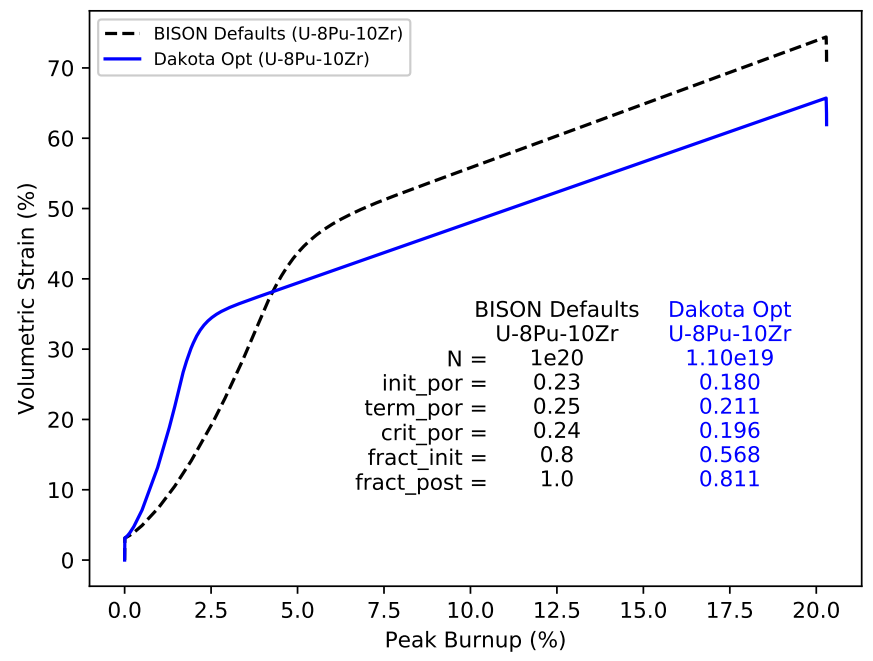

Figure 3.15: Comparison of volumetric strain versus burnup for the default values of the FGR parameters and the Dakota-optimized values for $\mathrm{U}-8 \mathrm{Pu}-10 \mathrm{Zr}$.

\subsection{Lower-Length-Scale-Informed Modeling}

To inform the BISON swelling and FGR models, the growth and interconnection of fission gas bubbles in $\mathrm{U}-(\mathrm{Pu})-\mathrm{Zr}$ fuel was simulated using the phase-field method [4]. The model tracks the normalized defect concentration as an order parameter, and the system's microstructure is evolved using the Cahn-Hilliard equation, with a source term added to represent the production of defects in the fuel matrix surrounding the bubbles. A set of initially spherical bubbles are placed in the simulated initial conditions at random positions, with a number density based on experimental micrographs taken before significant interconnection began. Samples of microstructural evolution are shown in Figure 3.16.

The phase-field simulations were used to determine values for the interconnection initiating and terminating porosity parameters in the BISON UPuZrGaseousEigenstrain swelling model. These values were determined to be 0.26 and 0.28 , respectively. The process to determine these values from the mesoscale simulations was described fully in [4]. In FY 2020, additional runs were conducted using the same methodology to obtain better statistics. The details of these simulations will be described further in a forthcoming report; however, the initial values of 0.26 and 0.28 were unchanged. These values of interconnection initiating and terminating porosity were used in another set of Dakota optimization runs, in which those parameters along with the critical porosity (i.e., typically the average of the initiating and terminating porosity values) were eliminated from the optimization process. The remaining parameters (bubble number density, initial and post-FGR fractions) were calibrated to the EBR-II FGR data through the optimization process. The results are shown in the following figures (3.17-3.19). 


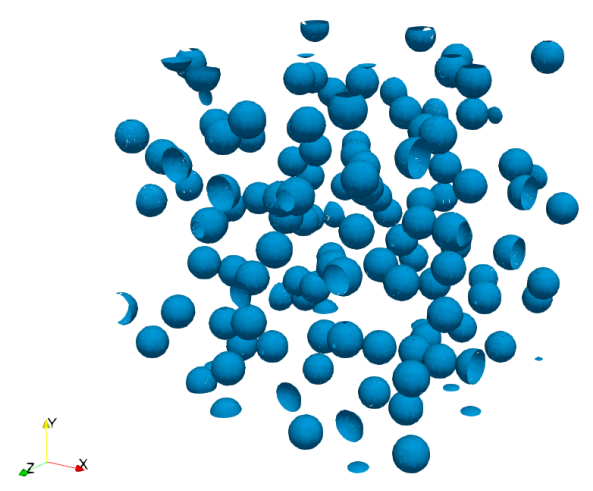

(a) Initial conditions. Porosity $p=4.5 \%$

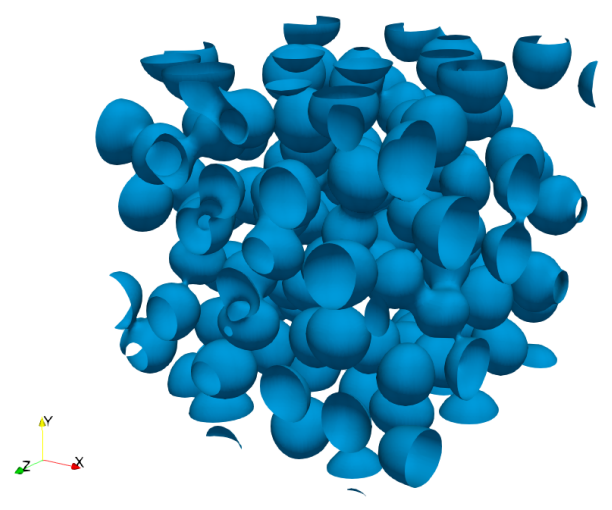

(c) $p=19.4 \%$

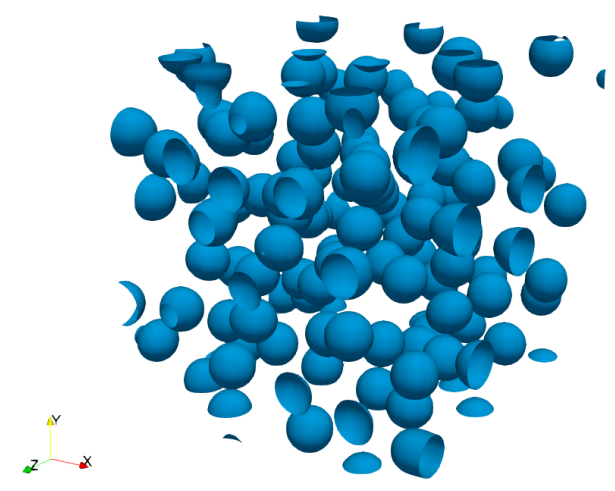

(b) $p=10.1 \%$

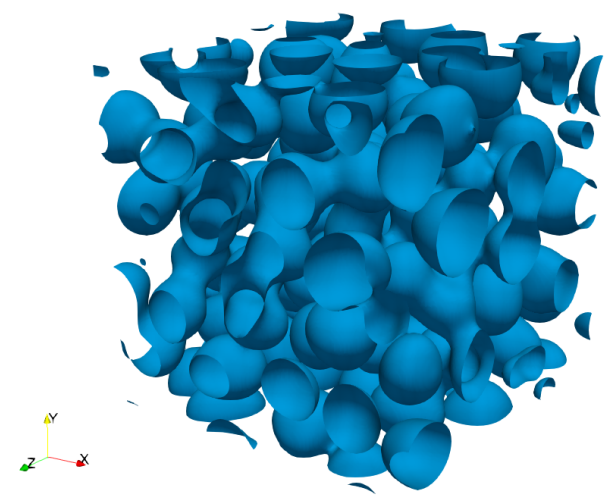

(d) $p=26.4 \%$

Figure 3.16: Evolution of microstructure during simulated gas bubble growth [4]: (a) initial conditions; (b) the bubbles have grown but not yet begun to merge; (c) bubble merging and coalescence has begun; (d) a percolated path exists from the simulation domain boundary at $x=0 \mu \mathrm{m}$ to the boundary at $x=72 \mu \mathrm{m}$. 


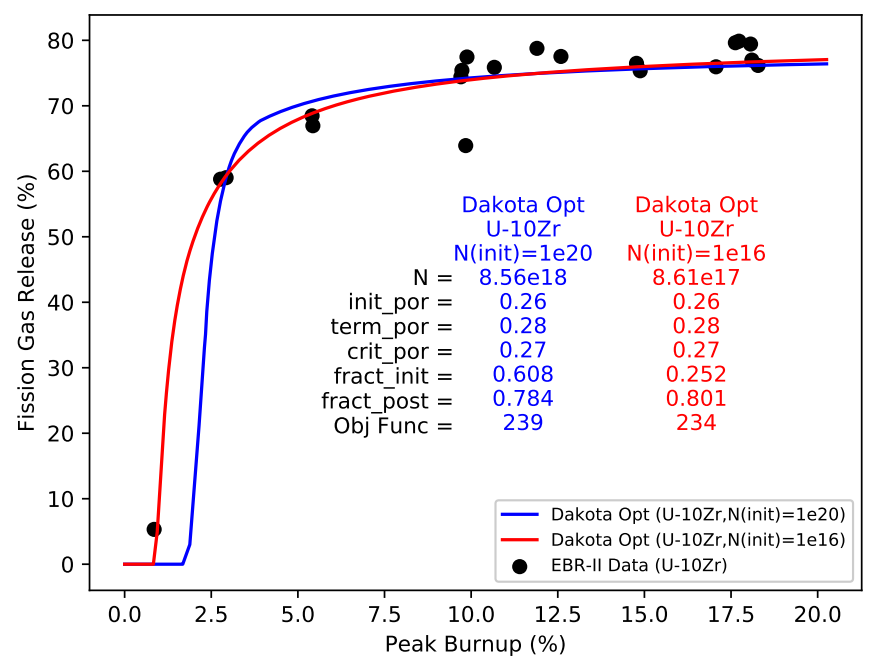

Figure 3.17: Comparison of optimized Dakota results using $\mathrm{N}=1 \mathrm{E} 20$ and $\mathrm{N}=1 \mathrm{E} 16$ as initial guesses in the LLSinformed BISON simulation for U-10Zr EBR-II metallic fuel.

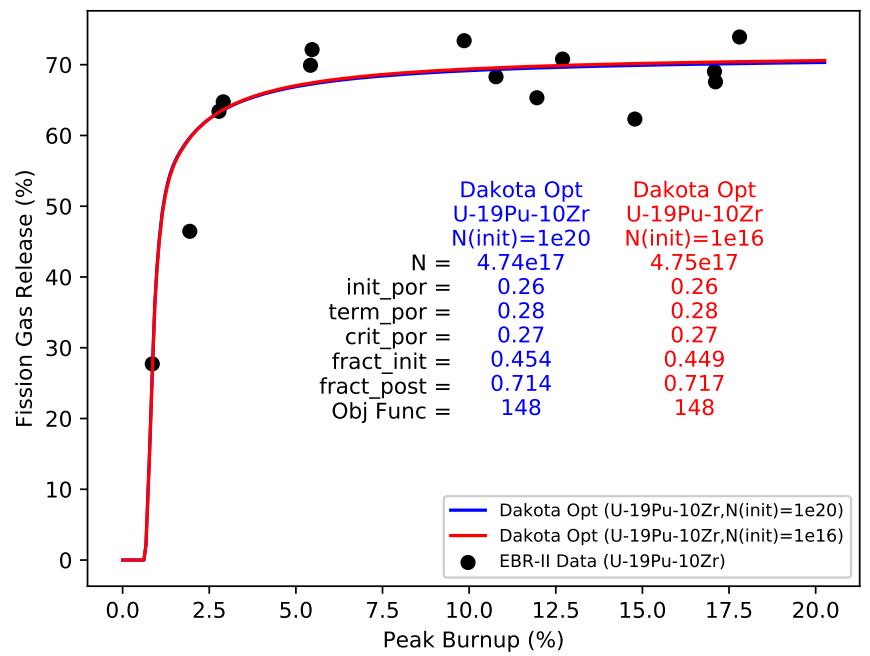

Figure 3.18: Comparison of optimized Dakota results using $\mathrm{N}=1 \mathrm{E} 20$ and $\mathrm{N}=1 \mathrm{E} 16$ as initial guesses in the LLSinformed BISON simulation for U-19Pu-10Zr EBR-II metallic fuel. 


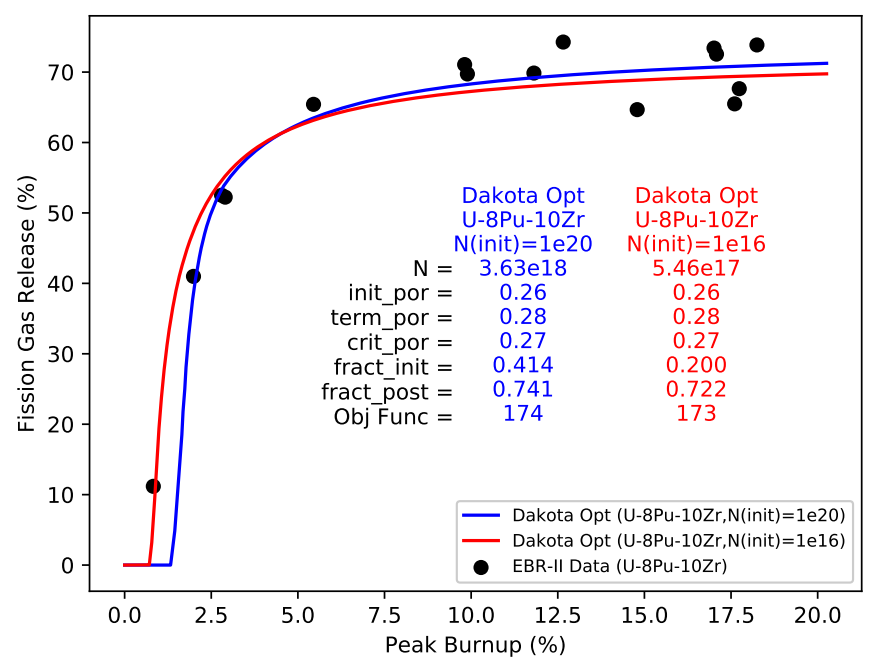

Figure 3.19: Comparison of optimized Dakota results using $\mathrm{N}=1 \mathrm{E} 20$ and $\mathrm{N}=1 \mathrm{E} 16$ as initial guesses in the LLSinformed BISON simulation for U-8Pu-10Zr EBR-II metallic fuel.

\subsection{Bubble Number Density}

An effort was made to correlate bubble number density with burnup for the EBR-II experiments using the available micrographs from historical PIE results. The quality of the images available was not ideal; therefore, the application of image analysis techniques to those micrographs was difficult. Several settings in the image analysis software related to thresholding, in addition to the algorithms used to differentiate porosity, could significantly change the results. Therefore, a fairly large scatter in the results (as seen in Figure 3.20) was observed. In addition, the simple assumption of spherical porosity was made when converting the porosity and number of pores into a bubble number density value. However, the trend of the bubble number density with burnup appears to be physical, although the range of values may be overestimated.

Correlation of the bubble number density with burnup was implemented in a BISON input file using a piecewiselinear function and a GenericFunctionMaterial type to transfer the value of $\mathrm{N}$ into the material property for use in the UPuZrGaseousEigenstrain model. The correlation was capped at $1 \mathrm{e} 22$ for the initial value of bubble number density at low burnup (i.e., less than 0.5\%). However, as seen in Figure 3.21, using this correlation delays FGR significantly. This is due to the gradual decrease of $\mathrm{N}$ with burnup. As a way to gauge the effect of different bubble number density functions, the value of $\mathrm{N}$ in the current correlation was decreased by 10 and 100 . The results of these simulations are shown in Figure 3.21, and they bring the FGR behavior in closer alignment with the data. 


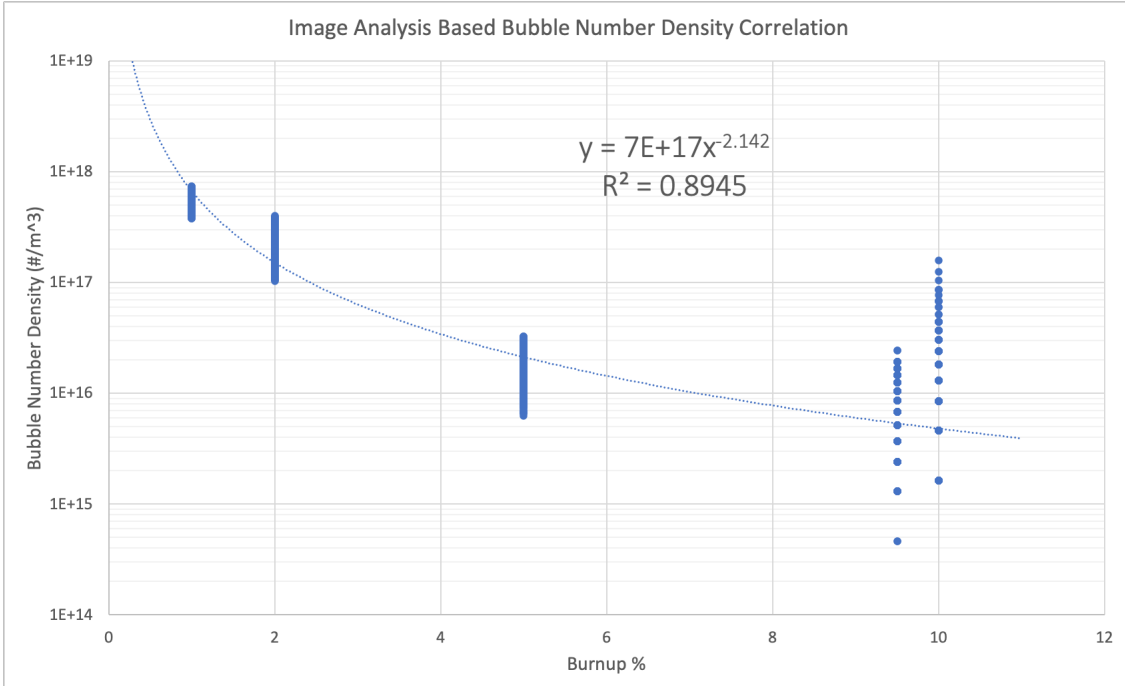

Figure 3.20: Bubble number density versus burnup from image analysis of selected micrographs and a best-fit powerlaw trendline equation.

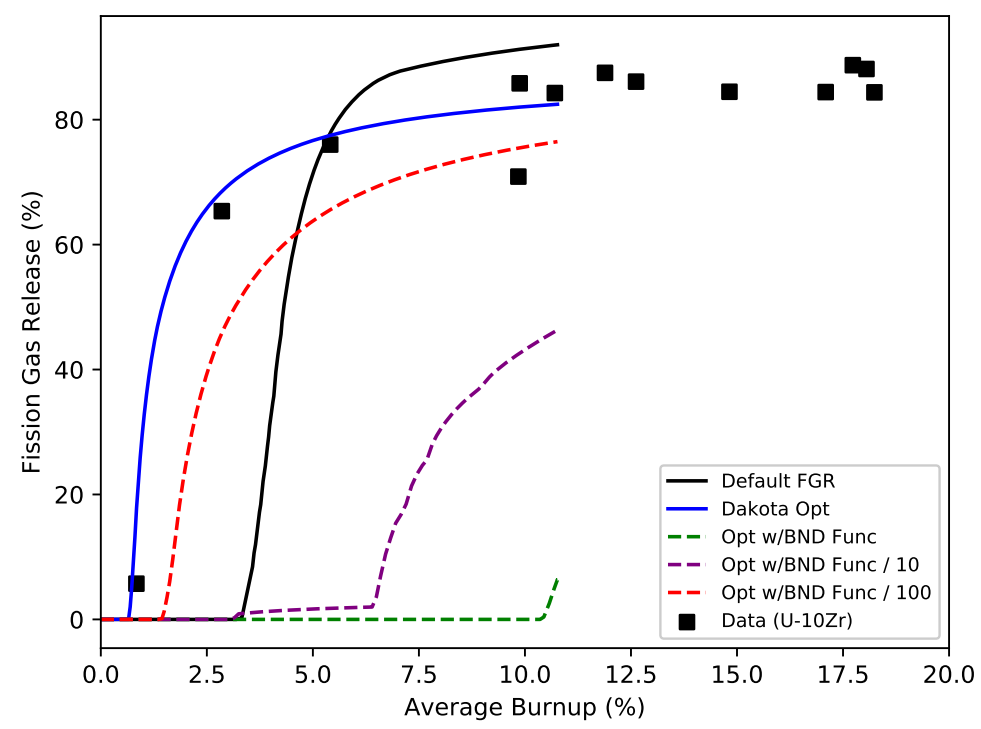

Figure 3.21: Comparison of BISON predictions for FGR using Dakota-optimized parameters and the bubble number density (BND Func) correlation from image analysis. 


\subsection{Anisotropic Swelling Optimization}

It is well-known that zirconium-based metallic fuel swells radially more than axially. Therefore, we attempted to apply the same optimization strategy to the anisotropic factor in the gaseous swelling model. This allows more swelling in the radial direction, as the value for the anisotropic factor is changed from zero to one. Figures 3.22 and 3.23 provide the basis of the EBR-II experimental data used in an attempt to calibrate the anisotropic factor.

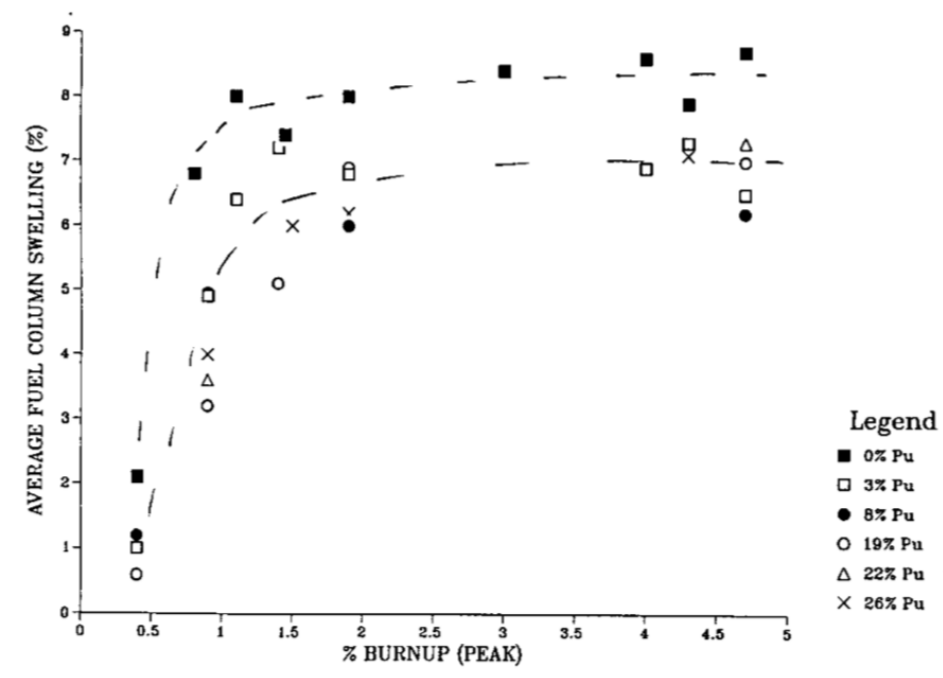

Fig. 1-Axial fuel swelling of 0.290 -in. elements.

Figure 3.22: Original plot of fuel axial strain data1 [5].

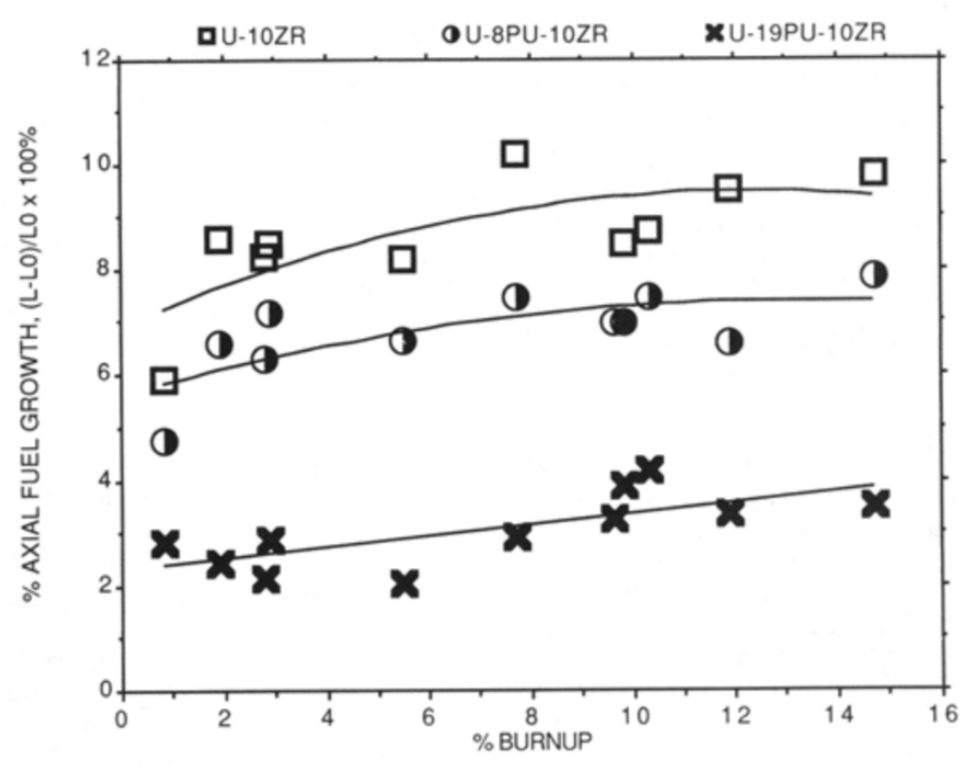

Figure 3.23: Original plot of fuel axial strain data2 [6].

A simple metallic fuel model was run with an initial porosity of zero, along with varying anisotropic factor values, to determine the effect of that parameter and compare the default (i.e., anisotropic_factor $=0$ ) behavior of BISON to 
the EBR-II experimental data. Figure 3.24 shows the results, illustrating that BISON overpredicts axial strain at higher burnup. This caused efforts to calibrate the anisotropic factor value using a procedure similar to that used for the FGR parameters to fail (by being unable to arrive at parameters that apply to both low and high burnup). However, using the low-burnup EBR-II data labeled "data1" and limiting the results to burnups of less than 5\% (Figure 3.25) indicates that an anisotropic factor value of 0.25 matches the data reasonably well. Figure 3.26 shows the effect of the initial porosity on axial strain and suggests that porosity values between zero and $5 \%$ are reasonable when comparing BISON to this subset of data. These results are preliminary and should be used cautiously until the anisotropic swelling model can be modified to include more physics to correct the overprediction at higher burnup.

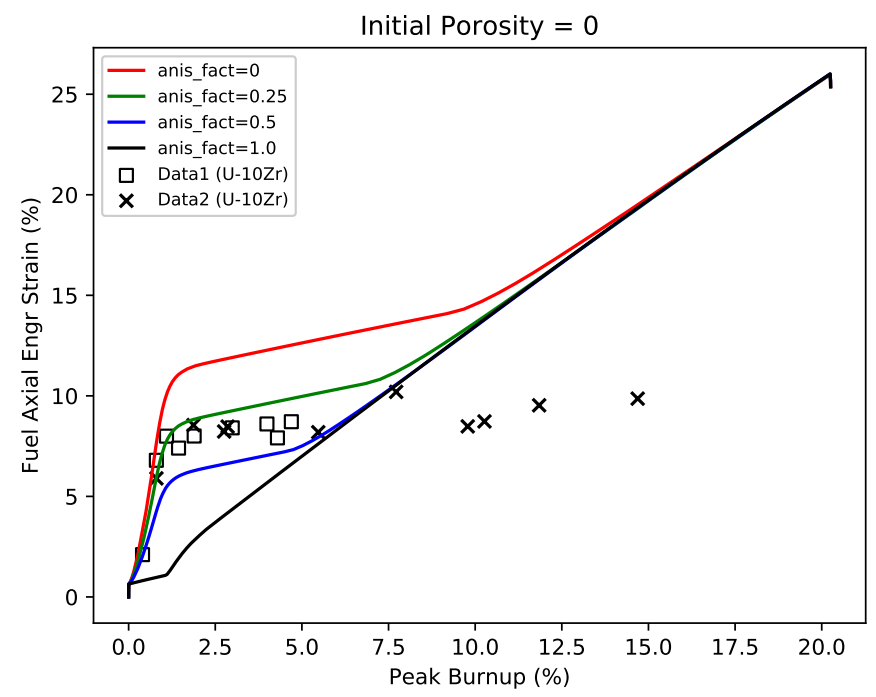

Figure 3.24: Initial comparison of the BISON fuel axial engineering strain and EBR-II experimental data. 


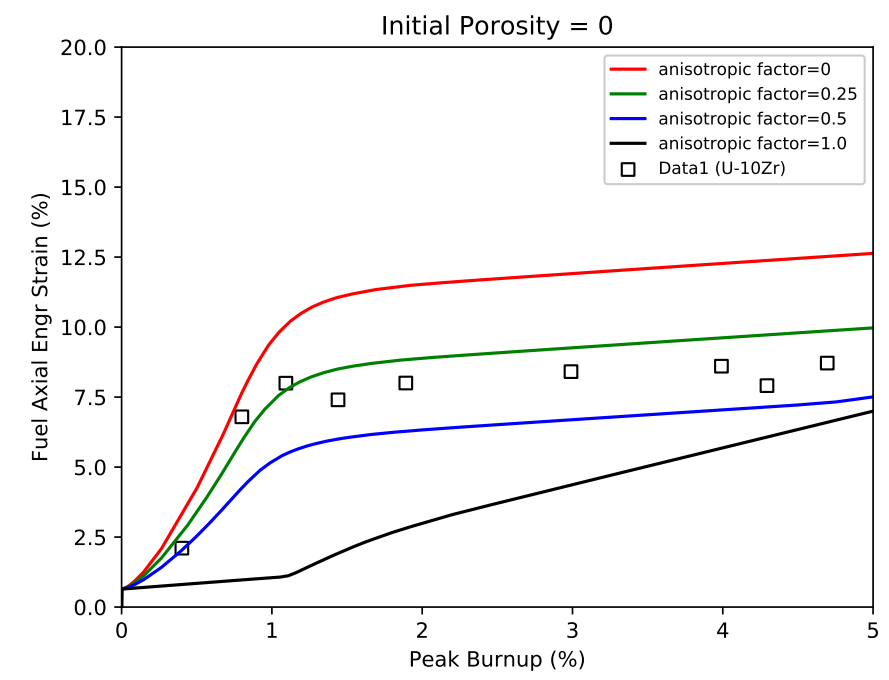

Figure 3.25: Effect of the anisotropic factor on the fuel axial strain results for the low-burnup range.

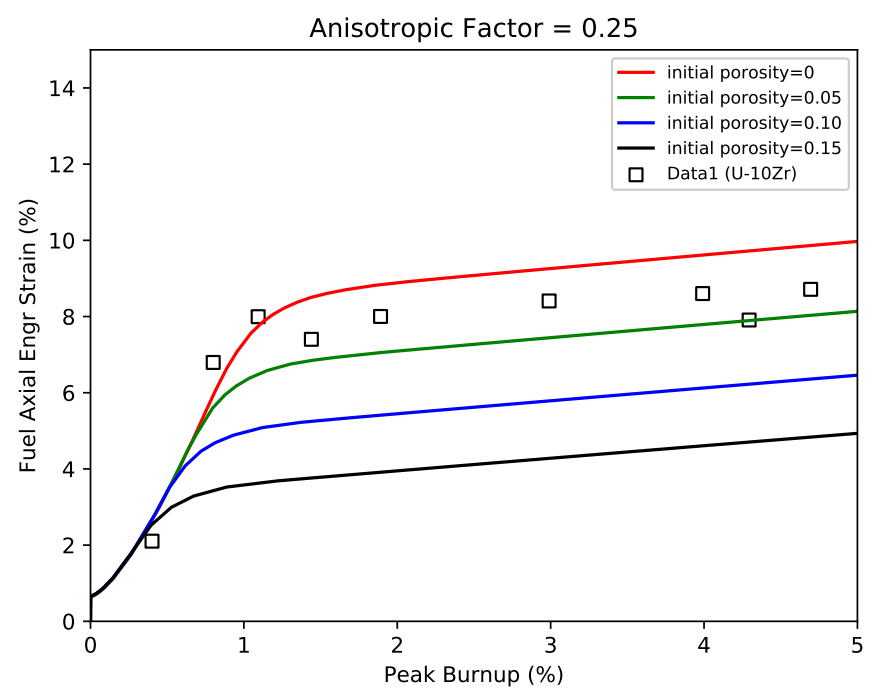

Figure 3.26: Effect of initial porosity on fuel axial strain results for the low-burnup range. 


\subsection{Discussion}

Calibration of the FGR parameters in BISON through an optimization process demonstrates the continuing improvement of BISON for modeling metallic fuel behavior. In addition, the LLS-informed results for the porosity interconnection phenonmenon were incorporated in engineering-scale simulations. The combination of lower-length-scale research and engineering-scale modeling provides a more robust tool for simulating metallic fuel performance in the evaluation of sodium-cooled fast-reactor designs.

Unfortunately, not all the work in this research project ended with as clear a conclusion as the FGR calibration. Specifically, the bubble number density and anisotropic swelling factor require additional research to resolve issues not anticipated when planning these tasks. However, by examining the BISON results obtained, some suggestions for improving the code within the current limitations can be made.

The anisotropic swelling behavior changes with increasing Pu content in the fuel (Figure 3.23). As the EBR-II data shows, fuel axial growth decreases as the Pu content in the fuel increases. Although BISON currently has limited capability for including the effect of Pu on the swelling behavior of the fuel, the anisotropic factor and initial porosity provide a means of simulating the same effect. As seen in 3.25 and 3.26, the predicted axial strain decreases when both the anisotropic factor and initial porosity are increased. These parameters could be used as a temporary means of accounting for the effect of $\mathrm{Pu}$ on fuel axial strain at the low range of burnup. A process similar to that used for the FGR calibration can be applied to the fuel axial growth data via an optimization of these parameters.

\subsection{Conclusions}

BISON metallic fuel FGR and swelling models have been improved through the use of lower-length-scale modeling results and the calibration of model parameters using EBR-II experimental data. The optimization process used to calibrate the BISON parameters is generic and can be applied to additional phenomena, provided that data is available and the objective function is reasonably sensitive to changes in the parameters. This improved swelling model is positively impacting the Versatile Test Reactor Program's scoping calculations work and the DOE-FOA-0001953 Next-Generation Metallic Fuel project at INL.

Additional work was performed in regard to bubble number density evolution and anisotropic swelling behavior in metallic fuel. Unfortunately, issues were encountered that prevented the results of those efforts from being incorporated into the BISON code and thereby improving the simulation of metallic fuel swelling. Suggestions are made in the next section to extend the work in those areas and potentially utilize future results within BISON.

\subsection{Future Work}

One area of potential future research is calibration of the anisotropic swelling behavior observed in metallic fuels. An increase in fuel axial strain occurs as the plutonium content of the fuel decreases. This effect could be captured using both the anisotropic factor and initial porosity parameters in the UPuZrGaseousEigenstrain material model, and by using the low-burnup EBR-II experimental data.

In addition, the optimization method used in this research was robust and fairly efficient; however, better methods may be available and should be investigated. Also, the current technique does not ensure finding a global minimum, as seen from the different results obtained when using different starting values for the bubble number density, especially in the U-19Pu-10Zr fuel composition. Determining a global minimum may not be possible in this case, but lessening the dependence of the solution on the initial values of the parameters used in the optimization should be a worthwhile goal.

The bubble number density evolution in metallic fuel could be better understood, and a more accurate correlation for bubble number density as a function of burnup or some other quantity should be developed. Efforts are being made in this area by the lower-length-scale group, and plans to incorporate the results of their research into the engineeringscale calculations should be prioritized.

Another development of note is the merging of a new viscoplastic-based swelling model into BISON later this FY. It computes an inelastic volumetric strain to account for gaseous swelling via a bubble surface force-balance model. This is a fundamentally different swelling model in that it is not an eigenstrain-based model and, as such, requires implementation into integrated fuel pin simulations and comparison with existing models and experiment measurements for proper evaluation. 
Additionally, Los Alamos National Laboratory (LANL) has work planned this FY that may be used as input to the work presented here. The LANL work package is titled "M3MS-20LA0201058 - Develop and demonstrate capability of new multi-phase mechanistic metallic fuel swelling model." The description of that milestone is as follows: "This milestone is a continuation of successes from FY-19 on development of a mechanistic swelling model that can describe fuel swelling, porosity interconnection, and fission gas release. FY-20 will focus on phase-specific properties, with an eye towards utilization of lower-length scale information such as gas diffusivity. Models will benefit from consistent viscoplasticity methods developed in FY-19, with application to the complex phase-specific properties. The resulting swelling model will be poised for implementation and testing in BISON, which will be done in collaboration with the team at INL." The due date for LANL's milestone is July 31, while the milestone presented here is due on June 12. It seems prudent to update the milestone presented here for the purpose of incorporating LANL's work later this FY.

Finally, although we proposed to compare our calculations to experiment measurements from the Advanced Fuels Campaign (AFC) program, we were unable to accomplish that due to the defunding of metallic-fuel-related work in the AFC program during FY-20. We plan to revisit this if funding is reestablished.

\subsection{Appendix}

To preserve the Dakota optimization approach, the process is documented here.

\subsubsection{Input Files}

The necessary input files required to run a Dakota optimization are:

- Dakota input file

- BISON-templated input file

- Shell script to set up the preprocessing, BISON simulation, and postprocessing for the Dakota process

- Python script to calculate the objective function for use in the optimization

- dprepro script.

\subsubsection{Running Process}

The basic process for Dakota optimization is as follows:

1. Create a working directory for the initial run using the initial point values.

2. Copy the files into the working directory and run dprepro on the BISON input file template to create the simulation.

3. Run BISON and generate results.

4. Run the objective function script to compute the objective function value.

5. Compute parameter variations for use in the next set of BISON simulations.

6. Create working directories for additional simulations and run them (using parallel execution from within Dakota).

7. Continue the process until the number of allowed simulations is reached, or the change in the objective function falls within the solution tolerance. 


\subsubsection{Dakota Input File}

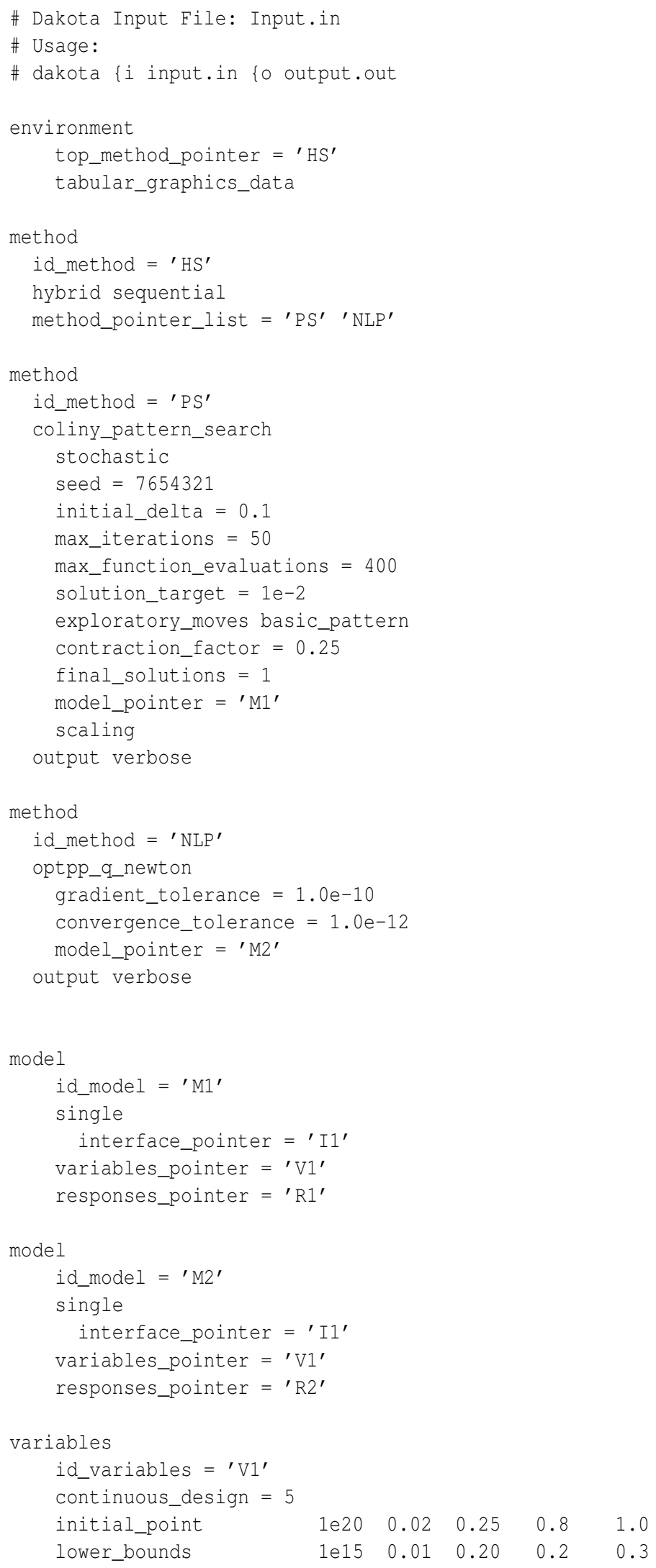




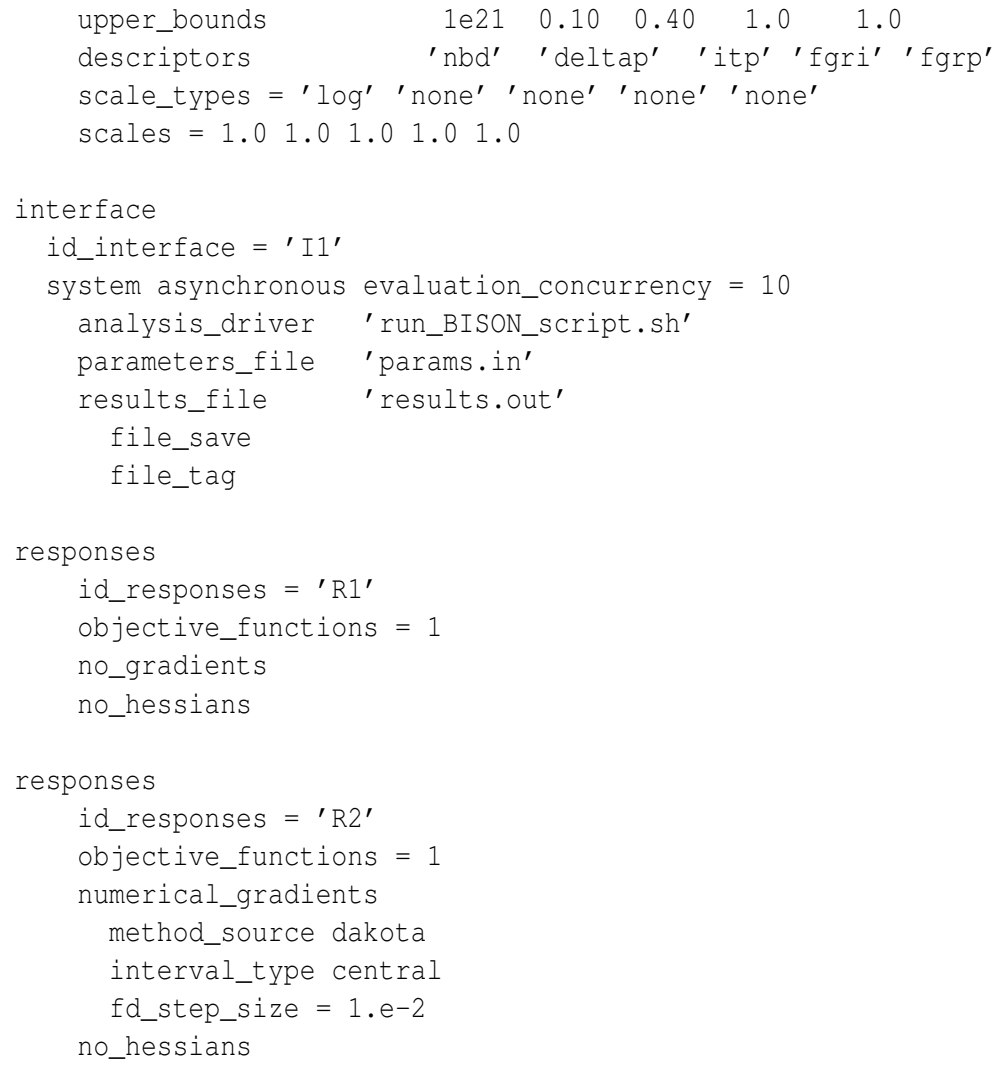

\subsubsection{BISON-Templated Input File}

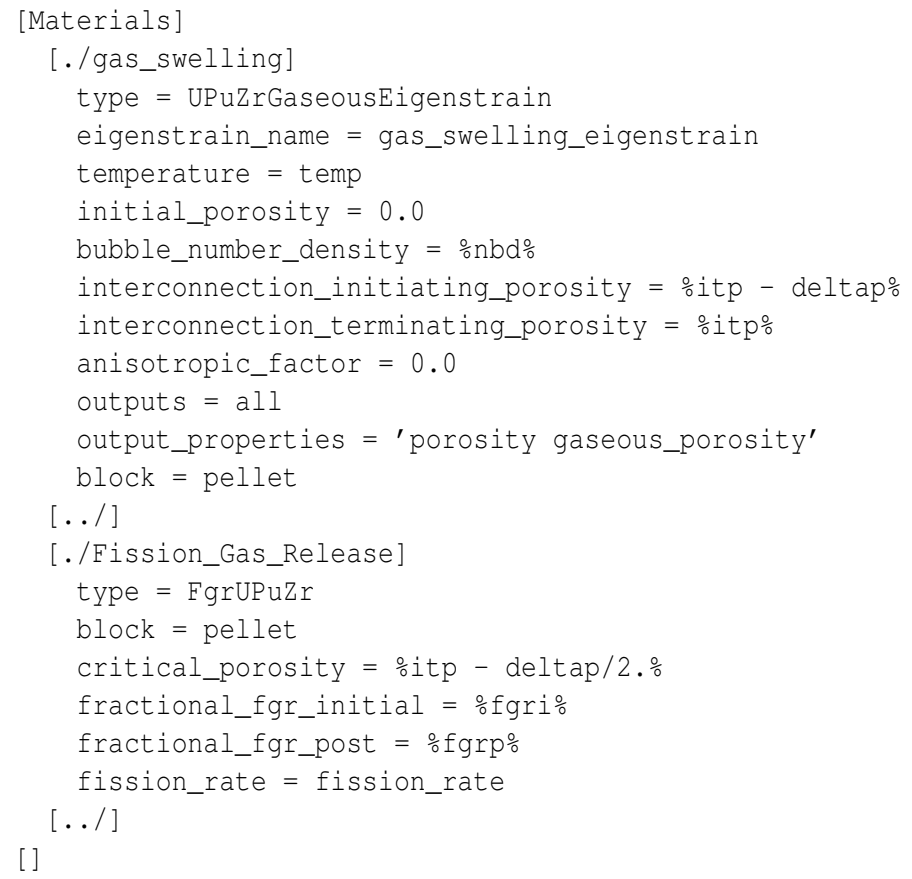

\subsubsection{Optimization Run Shell Script}

\#!/bin/bash 


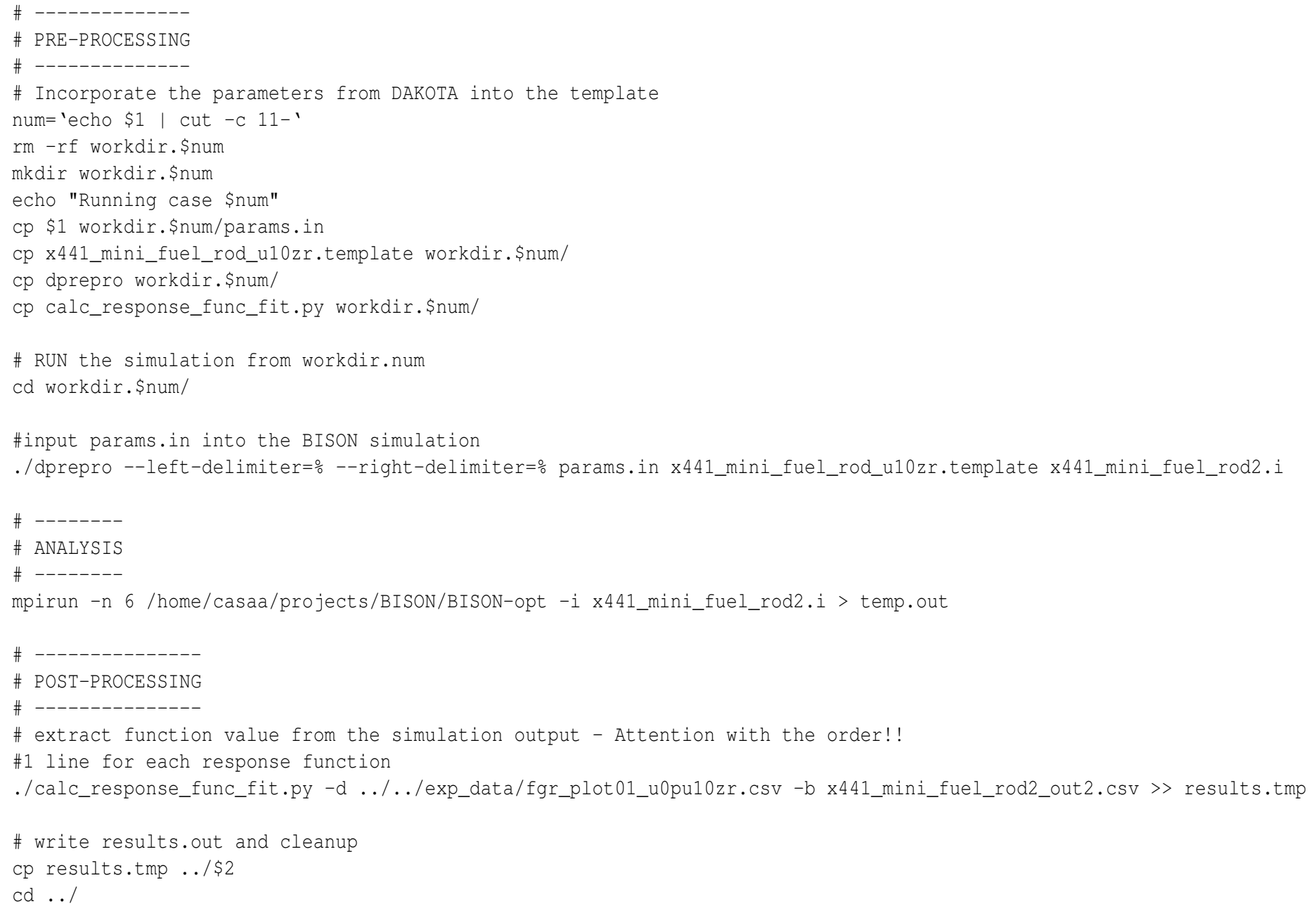

\subsubsection{Python Objective Function Script}

\#! /opt/moose/miniconda/bin/python

import pandas

import math

import argparse

def get_options():

" ""Command-line options."" "

parser = argparse.ArgumentParser (description=' calculate response function for FGR') 


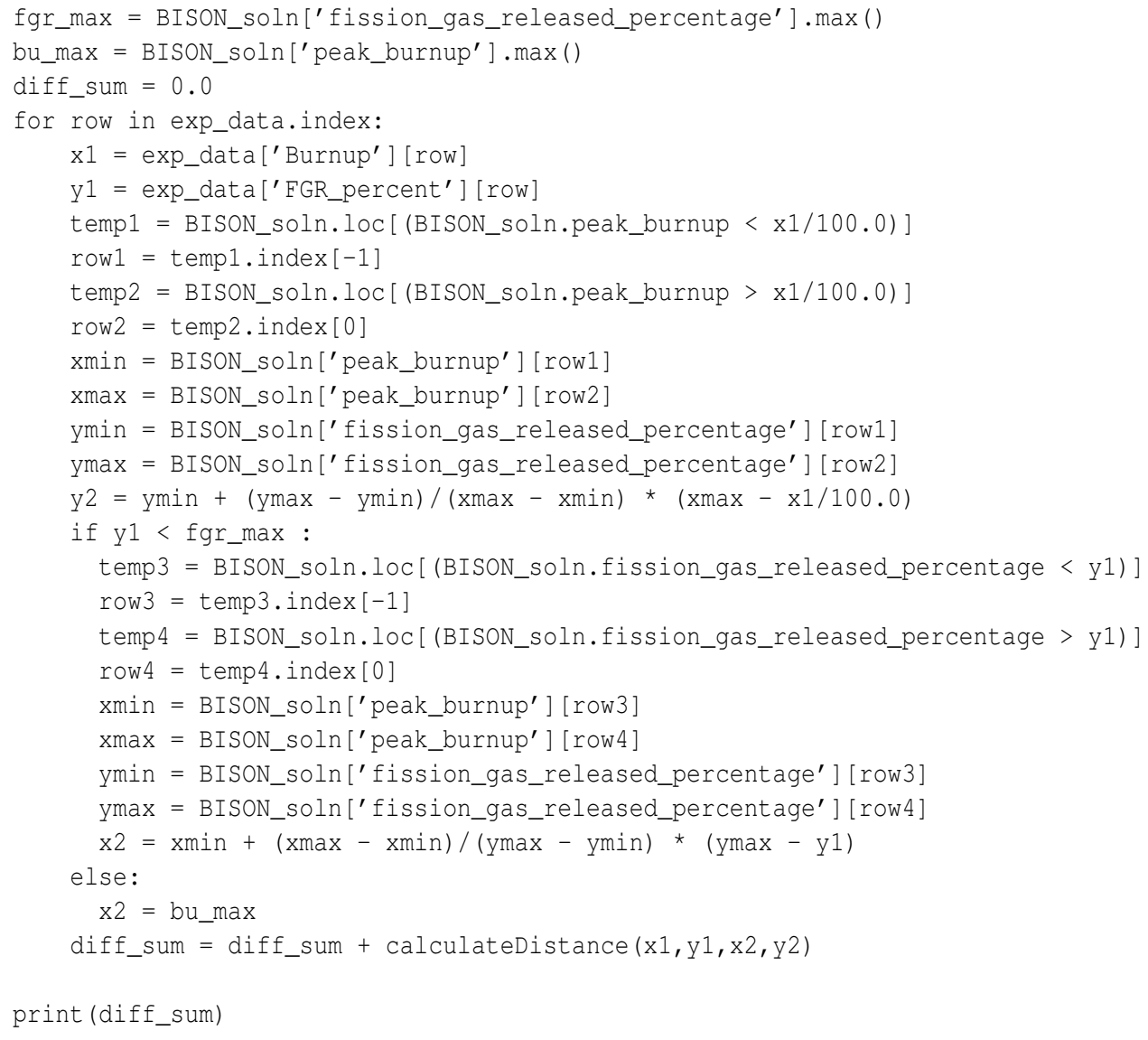

\subsubsection{Updated Anisotropic Swelling Calibration}

From the observations that the initial porosity and anisotropic swelling factor seemed to influence the anisotropic swelling predictions in the low burnup regime, the low burnup data (i.e., less than $\sim 6 \%$ ) was extracted and used as the basis of another set of Dakota optimization runs. Initially, both the anisotropic swelling factor and the initial porosity were used in the calibration. However, as can be seen in Figure 3.27 the starting values used in the optimizations did not converge to a single set of values for the final optimized results. Instead, depending on the initial values, the final values for the anisotropic swelling factor and initial porosity could be different but still provide a good final prediction compared to the data. Therefore, the two input variables were separated and the initial porosity was fixed at three values $(0 \%, 5 \%$, and $10 \%)$ for multiple calibration runs using different starting values for the anisotropic swelling factor.

\subsubsection{Varying Initial Porosity}

The same low burnup experimental data for $\mathrm{U}-10 \mathrm{Zr}$ was used with an initial porosity value set to $0 \%$. The initial value of the anisotropic swelling factor was set to $0,0.5$, and 1.0 to determine if the the optimization would converge to the same final value. As can be seen in Figure 3.28 the Dakota optimization process does converge although the agreement with the experimental data is not particularly good for the case where the initial porosity is zero. However, the results improve significantly when the initial porosity is non-zero, as can be seen in Figures 3.29 and 3.30 . In both cases, the results converge to a single value regardless of the initial value for the anisotropic swelling factor.

\subsubsection{Fuel Composition}

The EBR-II experiments were conducted for a range of fuel compositions and the anisotropic swelling data is available for $\mathrm{Pu}$ containing fuels. However, applying the same technique to the anisotropic swelling data for the U-19Pu-10Zr 


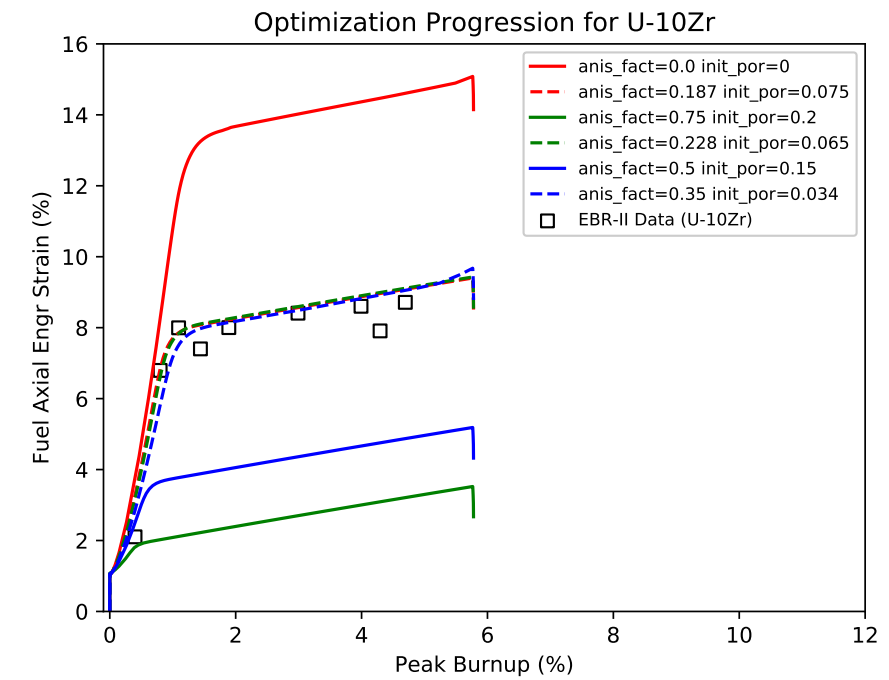

Figure 3.27: The starting and ending simulation results for anisotropic swelling calibration using the anisotropic swelling factor and initial porosity as variables in Dakota. Note: the solid lines are the starting values for the optimization runs and the dashed lines are the final optimized values.

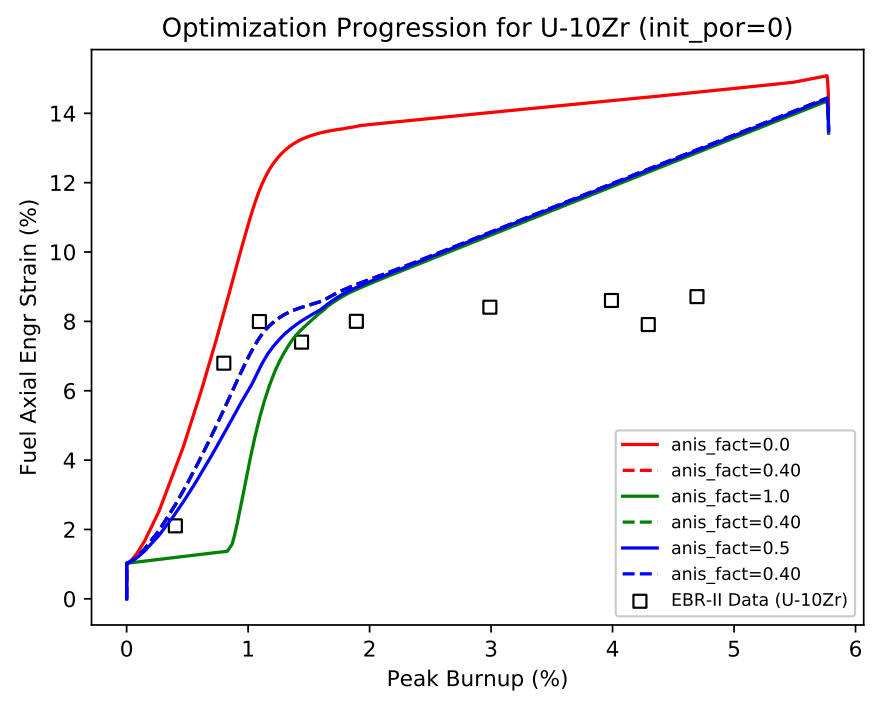

Figure 3.28: The starting and ending simulation results for anisotropic swelling calibration using a fixed value of $0 \%$ for the initial porosity while allowing the initial value of the anisotropic swelling factor to vary. Note: the solid lines are the starting values for the optimization runs and the dashed lines are the final optimized values. 


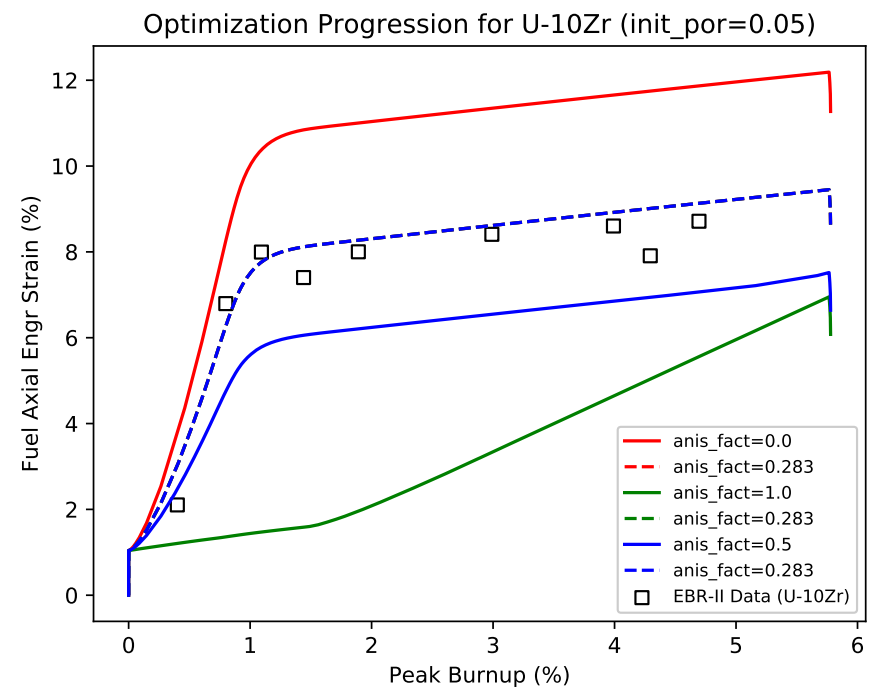

Figure 3.29: The starting and ending simulation results for anisotropic swelling calibration using a fixed value of 5\% for the initial porosity while allowing the initial value of the anisotropic swelling factor to vary. Note: the solid lines are the starting values for the optimization runs and the dashed lines are the final optimized values.

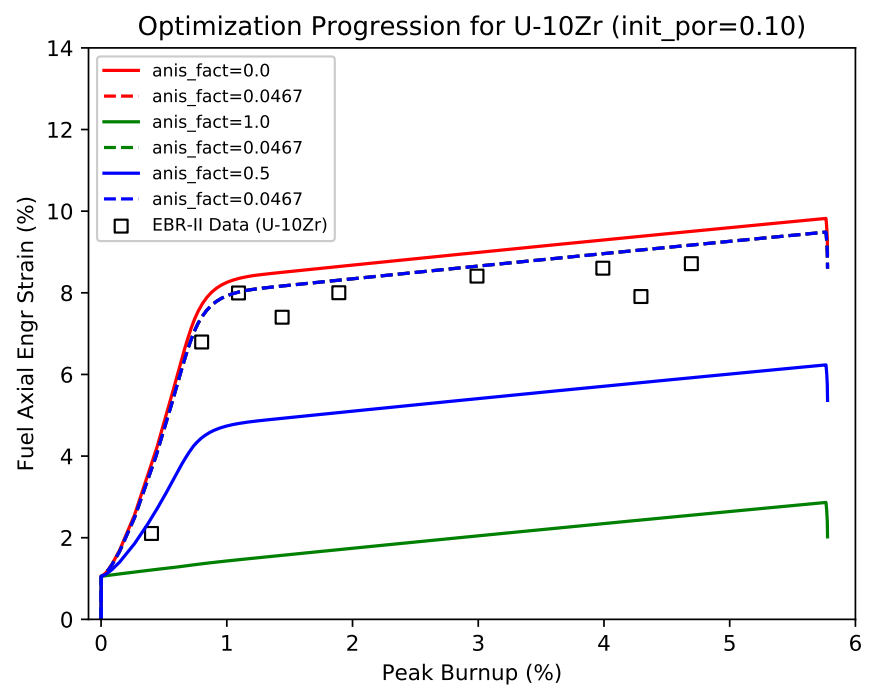

Figure 3.30: The starting and ending simulation results for anisotropic swelling calibration using a fixed value of 5\% for the initial porosity while allowing the initial value of the anisotropic swelling factor to vary. Note: the solid lines are the starting values for the optimization runs and the dashed lines are the final optimized values. 
fuel did not produce similar results. Figure 3.31 shows the initial results using both the initial porosity and anisotropic swelling factor as variables in the Dakota optimization. Once again, the converged values of the variables do not agree and in this case the agreement of the optimized results and the data is not as good. Unfortunately, the approach of separating the variables and fixing the initial porosity used for the $\mathrm{U}-10 \mathrm{Zr}$ fuel above, did not improve the agreement between the optimized results and the data. Therefore, the attempt to calibrate the current anisotropic swelling model is BISON has been concluded. Clearly, a more sophisticated model of the anisotropic swelling behavior is needed in order to fully explain the EBR-II experimental data and should be the focus of future work in metallic fuels.

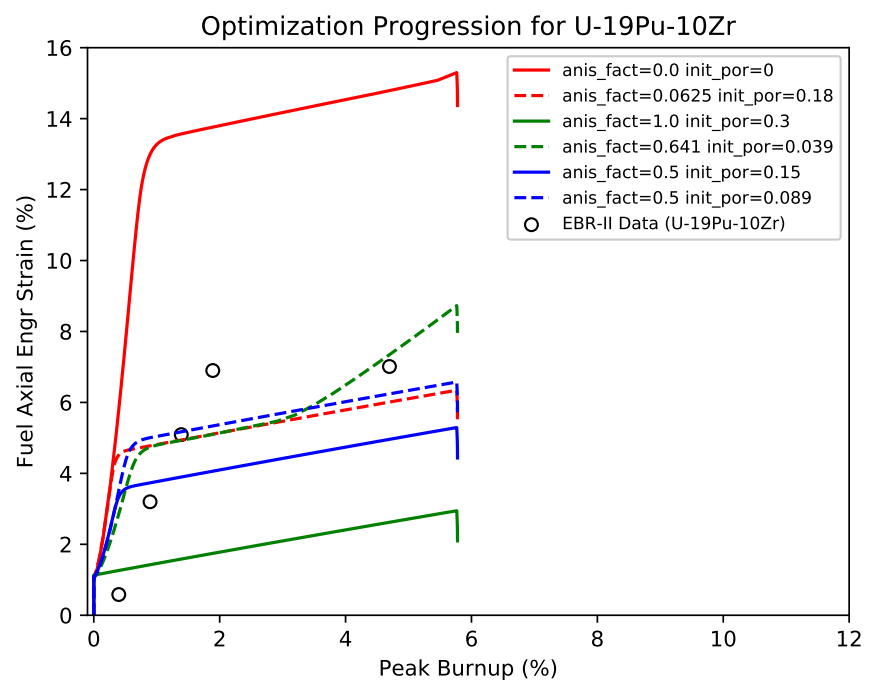

Figure 3.31: The starting and ending simulation results for anisotropic swelling calibration of $\mathrm{U}-19 \mathrm{Pu}-10 \mathrm{Zr}$ fuel using the anisotropic swelling factor and initial porosity as variables in Dakota. Note: the solid lines are the starting values for the optimization runs and the dashed lines are the final optimized values. 


\section{Bibliography}

[1] R. G. Pahl, D. L. Porter, D. C. Crawford, and L. C. Walters. Irradiation behavior of metallic fast reactor fuels. Journal of Nuclear Materials, 188:3-9, 1992.

[2] A. M. Yacout, A. Oaks, W. Mohamed, and K. Mo. FIPD: EBR-II fuels irradiation \& physics database. Technical report, ANL-ART-124, Argonne National Laboratory, 2017.

[3] B. M. Adams, M. S. Ebeida, M. S. Eldred, G. Geraci, J. D. Jakeman, K. A. Maupin, J. A. Monschke, Stephens J. A., L. P. Swiler, D. M. Vigil, T. M Wildey, W. J. Bohnhoff, K. R. Dalbey, J. P. Eddy, J. R. Frye, R. W. Hooper, K. T. Hu, P. D. Hough, M. Khalil, E. M. Ridgway, and A. Rushdi. DAKOTA: A multilevel parallel object-oriented framework for design optimization, parameter estimation, uncertainty quantification, and sensitivity analysis. Version 6.7 user's manual. Technical Report SAND2014-4633, Sandia National Laboratories, 2017.

[4] L. K. Aagesen, B. W. Beeler, A. Casagranda, A. M. Jokisaari, S. R. Novascone, A. Rezwan, M. R. Tonks, and Y. Zhang. Marmot modeling of swelling in U-Zr and integration into BISON. Report INL/EXT-19-55959, Idaho National Laboratory, 2019.

[5] Gerard L. Hofman, R. G. Pahl, C. E. Lahm, and D. L. Porter. Swelling Behavior of U-Pu-Zr Fuel. Metallurgical Transactions A, 21A:517-528, 1990.

[6] R. G. Pahl, D. L. Porter, C. E. Lahm, and G. L. Hofman. Experimental Studies of U-Pu-Zr Fast Reactor Fuel Pins in EBR-II. Technical Report CONF-8809202-2, Argonne National Laboratory, 1988. 


\section{Chapter 4}

\section{DEMONSTRATE BISON CAPABILITY FOR HTGR FUEL APPLICATIONS W. Jiang \\ J.D. Hales \\ A. Toptan \\ K.A. Gamble \\ S.R. Novascone \\ X.-Y. Liu \\ C. Matthews}

The purpose of this report is to document development of modeling TRistructural ISOtropic (TRISO) fuel particles. First, existing TRISO uranium oxycarbide (UCO, a two-phase mixture of $\mathrm{UO}_{2}$ and $\mathrm{UC}_{2}$ ) thermal and mechanical models from PARFUME[1] were implemented in BISON. They include elastic, creep, swelling, thermal expansion, and thermal conductivity models. All the models have corresponding regression tests and documentation. Secondly, a new fission gas release (FGR) model for UCO fuel kernel was added to BISON. This model incorporates effective diffusion coefficients from lower length scale calculations conducted by LANL. Lastly, prior work on statistical failure modes were extended to higher dimensions. The SiC failure due to IPyC cracking and particle asphericity are considered in failure determination. Several benchmark problems were performed to demonstrate BISON's capability for HTGR fuel applications.

\subsection{TRISO UCO Material Models}

TRISO fuel thermo-mechanical properties for the UCO kernel and coating layers are listed in this section. The properties include elastic, creep, swelling, thermal expansion, and thermal conductivity.

\subsubsection{UCO fuel kernel}

Legacy TRISO fuel development programs have predominantly focused on $\mathrm{UO}_{2}$ fuel and, consequently, most UCO kernel properties are derived from experimental data on $\mathrm{UO}_{2}$ and assumed valid for UCO due to lack of relevant $\mathrm{UCO}$ data.

The thermal conductivity of the kernel, $k(\mathrm{~W} / \mathrm{m}-\mathrm{K})$, is given by [2]:

$$
k=0.0132 \exp \left(0.00188 T_{C}\right)+ \begin{cases}\frac{4040}{464+T_{C}} & \text { for } T_{C}<1650^{\circ} \mathrm{C} \\ 1.9 & \text { for } T_{C} \geq 1650^{\circ} \mathrm{C}\end{cases}
$$

where $T_{C}\left({ }^{\circ} \mathrm{C}\right)$ is the temperature of the kernel.

The specific heat capacity of UCO is calculated from its molar heat capacity [3] and its molar mass:

$$
c_{P}=\frac{1}{M}\left(52.1743+87.951 t_{K}-84.2411 t_{K}^{2}+31.542 t_{K}^{3}-2.6334 t_{K}^{4}-\frac{0.71391}{t_{K}^{2}}\right)
$$


where $c_{P}(\mathrm{~J} / \mathrm{kg}-\mathrm{K})$ is specific heat capacity, $t_{K}=T_{K} / 1000(\mathrm{~K})$ is the reduced temperature, and $M(\mathrm{~kg} / \mathrm{mol})$ is the molar mass. The molar mass of the kernel, $M(\mathrm{~kg} / \mathrm{mol})$, is computed for $i=\mathrm{UO}_{2}, \mathrm{UC}_{2}$, and $\mathrm{UC}$ by

$$
M=\sum_{i}^{N} a f_{i} \times a w_{i}
$$

with

and

$$
a f= \begin{cases}0.5 \mathrm{OU} & \text { for } \mathrm{UO}_{2} \\ 0.5 O U+C U-1.0 & \text { for } \mathrm{UC}_{2} \\ 2.0-(O U+C U) & \text { for } \mathrm{UC}\end{cases}
$$

$$
a w= \begin{cases}0.23504 \varepsilon+0.23805(1.0-\varepsilon)+0.03200 & \text { for } \mathrm{UO}_{2} \\ 0.23504 \varepsilon+0.23805(1.0-\varepsilon)+0.02402 & \text { for } \mathrm{UC}_{2} \\ 0.23504 \varepsilon+0.23805(1.0-\varepsilon)+0.01201 & \text { for } \mathrm{UC}^{2}\end{cases}
$$

where $\varepsilon$ (wt.\%) is the initial U-235 enrichment, $O U$ (-) and $C U(-)$ are the initial oxygen to uranium and carbon to uranium stoichiometries of $\mathrm{UCO}$, af (-) is the atomic fraction, and $a w(\mathrm{~kg} / \mathrm{mol})$ is the atomic weight. The atomic weights of the UCO constituents are tabulated in Table 4.1.

Table 4.1: Atomic weights of the UCO constituents.

\begin{tabular}{ccccc}
\hline & uranium-235 & uranium-238 & oxygen & carbon \\
\hline$a w(\mathrm{~g} / \mathrm{mol})$ & 235.04 & 238.05 & 16.00 & 12.01 \\
\hline
\end{tabular}

The Thermal expansion coefficient is $10 \times 10^{-6}(1 / \mathrm{K})$ [4].

The Young's modulus of the kernel, $E$ (GPa), is given by [4]:

$$
E=219\left(1.0-1.1 \times 10^{-4} T_{C}-2.1 \times 10^{-7} T_{C}^{2}+3.1 \times 10^{-10} T_{C}^{3}-1.6 \times 10^{-13} T_{C}^{4}\right)\left(\frac{1.92 \rho-0.92 \rho_{t h}}{\rho_{t h}}\right)
$$

where $E(\mathrm{GPa})$ is the Young's modulus of the kernel, $T_{C}\left({ }^{\circ} \mathrm{C}\right)$ is the temperature of the kernel, $\rho\left(\mathrm{g} / \mathrm{cm}^{3}\right)$ is the density of the kernel, and $\rho_{t h}\left(\mathrm{~g} / \mathrm{cm}^{3}\right)$ is the theoretical density of UCO.

The theoretical density of the kernel, $\rho_{t h}\left(\mathrm{~kg} / \mathrm{m}^{3}\right)$ is calculated as

$$
\begin{gathered}
\rho_{t h}=\frac{1}{\frac{w f_{U O_{2}}}{\rho_{t h}^{U O_{2}}}+\frac{w f_{U C}}{\rho_{t h}^{U C}}+\frac{w f_{U C_{2}}}{\rho_{t h}^{U C_{2}}}} \\
w f=\frac{a f \cdot a w}{M}
\end{gathered}
$$

where $w f(-)$ is the weight fraction. The theoretical densities of the UCO mixture components are tabulated in Table 4.2.

Table 4.2: The theoretical densities of the UCO mixture components.

\begin{tabular}{cccc}
\hline & $\mathrm{UO}_{2}$ & $\mathrm{UC}$ & $\mathrm{UC}_{2}$ \\
\hline$\rho_{t h}\left(\mathrm{~g} / \mathrm{cm}^{3}\right)$ & 10.96 & 13.63 & 11.28 \\
\hline
\end{tabular}

Poisson's ratio of the kernel, $v(-)$, is given by:

$$
v=1.35\left(\frac{1.92 \rho-0.92 \rho_{t h}}{1.66 \rho-0.66 \rho_{t h}}\right)-1
$$

where $\rho\left(\mathrm{g} / \mathrm{cm}^{3}\right)$ is the density of the kernel and $\rho_{t h}\left(\mathrm{~g} / \mathrm{cm}^{3}\right)$ is the theoretical density of UCO. 
Kernel swelling occurs throughout irradiation as solid and gaseous atoms released by fission accumulate in the kernel, resulting in a volume increase of the kernel. The volumetric fission-induced swelling model of UCO is given by [5] and it assumes a constant rate of volume increase due to fission:

$$
\Delta \varepsilon_{s w}=0.8 \Delta B u
$$

where $\Delta \varepsilon_{s w}$ (-) is the volumetric swelling increment of the kernel and $\Delta B u$ (fissions/atoms- $\mathrm{U}$ ) is the burnup increment.

\subsubsection{Buffer}

The thermal conductivity of the buffer is given by [5]:

$$
k=\frac{k_{\text {init }} k_{\text {theo }} \rho_{\text {theo }}\left(\rho_{\text {theo }}-\rho_{\text {init }}\right)}{k_{\text {theo }} \rho_{\text {theo }}\left(\rho_{\text {theo }}-\rho\right)+k_{\text {init }} \rho\left(\rho-\rho_{\text {init }}\right)},
$$

where $k(\mathrm{~W} / \mathrm{m}-\mathrm{K})$ is the thermal conductivity of the buffer and $\rho\left(\mathrm{kg} / \mathrm{m}^{3}\right)$ is the density of the buffer. The values at initial $\left(\rho_{\text {init }}\right)$ and theoretical $\left(\rho_{\text {theo }}=2250 \mathrm{~kg} / \mathrm{m}^{3}\right)$ densities are from [6].

The initial thermal conductivity $\left(k_{\text {init }}\right)$ of the buffer is set to $0.5 \mathrm{~W} / \mathrm{m}-\mathrm{K}$. It is intended for an initial density of the buffer of $1000 \mathrm{~kg} / \mathrm{m}^{3}$, but it is also used for slightly different densities $( \pm 10 \%)$. The thermal conductivity of the buffer at its theoretical density $\left(k_{\text {theo }}\right)$ is set to $4.0 \mathrm{~W} / \mathrm{m}-\mathrm{K}$.

The specific heat capacity of the buffer is given by [7] as $720 \mathrm{~J} / \mathrm{kg}-\mathrm{K}$.

The isotropic coefficient of thermal expansion of the buffer is given by [5, 6]:

$$
\alpha=5\left(1+0.11\left[\frac{T_{C}-400}{700}\right]\right)
$$

where $T_{C}\left({ }^{\circ} \mathrm{C}\right)$ is the temperature of the buffer. The thermal expansion strain is calculated as:

$$
\varepsilon_{t e}=\alpha\left(T_{K}-T_{\text {StressFree }}\right)
$$

where $T_{K}(\mathrm{~K})$ is the temperature of the buffer, and $T_{\text {StressFree }}(\mathrm{K})$ is the stress-free temperature.

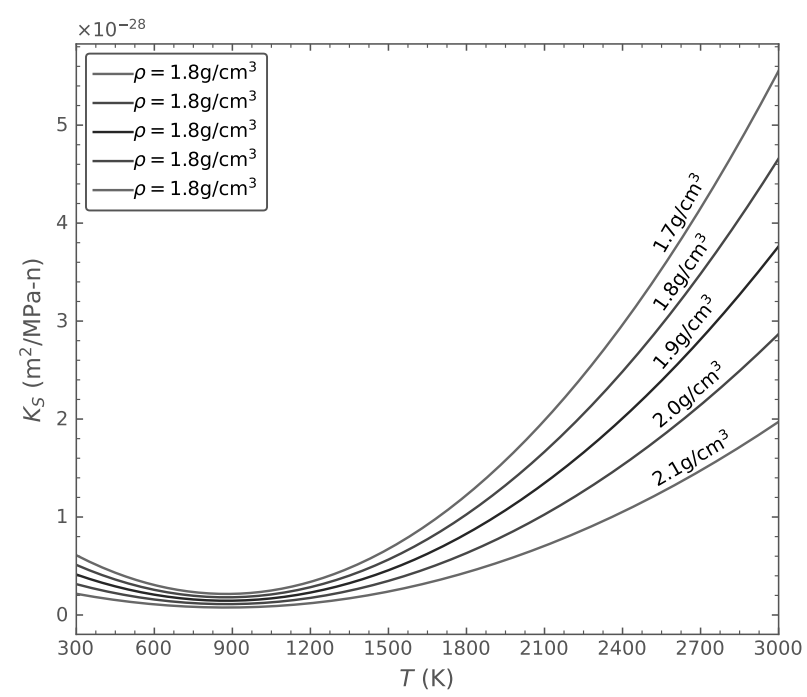

Figure 4.1: The irradiation-induced creep coefficient.

The Young's modulus of the buffer, $E$ (GPa), is given by [5, 6]:

$$
E=25.5\left(0.384+0.32410^{-3} \rho\right)(1.0+0.23 \phi)\left(1.0+1.510^{-4}\left[T_{C}-20\right]\right)
$$

where $\rho\left(\mathrm{kg} / \mathrm{m}^{3}\right)$ is the density of the buffer, $\phi\left(10^{25} \mathrm{n} / \mathrm{m}^{2}, \mathrm{E}>0.18 \mathrm{MeV}\right)$ is the fast neutron fluence, and $T_{C}\left({ }^{o} \mathrm{C}\right)$ is the temperature of the buffer. 
Poisson's ratio of the buffer is set to a constant value of $0.33[5,6]$.

The irradiation creep of the buffer and $\mathrm{PyC}$, that leads to its gradual deformation in response to irradiation-induced damage, is directly proportional to fast neutron fluence. The irradiation creep correlation for the buffer and PyC is taken from $[8,9,10]$. With $K\left(\mathrm{~m}^{2} / \mathrm{MPa}-\mathrm{n}\right)$ as the creep constant, $\sigma_{i}$ as one component of the principal stress, $\mathrm{v}_{c}$ as Poisson's ratio for creep, and $\Phi\left(10^{25} \mathrm{n} / \mathrm{m}^{2}, E>0.18 \mathrm{MeV}\right)$ as the fast neutron fluence, the creep rate is computed as

$$
\dot{\varepsilon}_{1}=K\left[\sigma_{1}-v_{c}\left(\sigma_{2}+\sigma_{3}\right)\right] \dot{\Phi}
$$

where

$$
K=K_{0}[1+2.38(1.9-\rho)] M_{\text {Irr, Creep }}
$$

The steady state creep coefficient, $K_{0}$ is given by [5] as

$$
K_{0}=2.193 \times 10^{-29}-4.85 \times 10^{-32} T+4.0147 \times 10^{-35} T^{2}
$$

where $M_{\text {Irr,Creep }}=2, \rho$ is in $\mathrm{g} / \mathrm{cm}^{3}$, and $T$ is in ${ }^{\circ} \mathrm{C}$ and bounded by $600^{\circ} \mathrm{C}$ and $1350^{\circ} \mathrm{C}$. The irradiation-induced creep coefficient is plotted at different temperatures and densities in Figure 4.1.

Poisson's ratio in creep of the buffer is set to a constant value of $0.5[5,6]$.

The buffer experiences isotropic irradiation-induced strain given by [5, 6]:

$$
\varepsilon_{i s o}=a_{1} \phi+a_{2} \phi^{2}+a_{3} \phi^{3}+a_{4} \phi^{4}
$$

where $\phi\left(10^{25} \mathrm{n} / \mathrm{m}^{2}, \mathrm{E}>0.18 \mathrm{MeV}\right)$ is the fast neutron fluence and $a_{1}$ through $a_{4}$ are temperature-dependent polynomial coefficients given in Figure 4.2. The polynomial coefficients at other temperatures are interpolated from Figure 4.2.

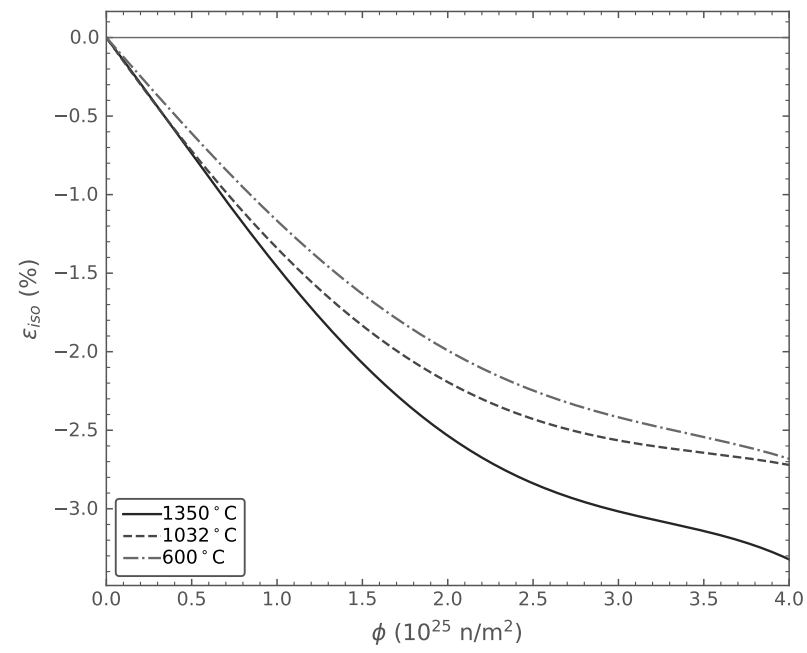

Figure 4.2: The buffer isotropic strain at a density of $1.96 \mathrm{~g} / \mathrm{cm}^{3}$. Polynomial coefficients for isotropic strain components are tabulated below in Table 4.3 .

Table 4.3: Polynomial coefficients at density of $1.96 \mathrm{~g} / \mathrm{cm}^{3}$.

\begin{tabular}{ccccc}
\hline Temp $\left({ }^{\circ} \boldsymbol{C}\right)$ & $\mathbf{a}_{1}$ & $\mathbf{a}_{2}$ & $\mathbf{a}_{3}$ & $\mathbf{a}_{4}$ \\
\hline 1350 & -1.42840 & -0.19563 & 0.18991 & -0.02591 \\
1032 & -1.52390 & 0.13048 & 0.06299 & -0.01072 \\
600 & -1.24080 & 0.00175 & 0.08533 & -0.01253 \\
\hline
\end{tabular}

For other densities $\varepsilon_{i s o}$ is adjusted by applying a multiplier given as:

$$
\mu=\frac{f(\rho)}{f\left(\rho_{0}=1.96\right)}
$$


where $\rho\left(\mathrm{g} / \mathrm{cm}^{3}\right)$ is the density of the buffer.

The isotropic density scaling factor is calculated using the values in Table 4.4, which shows the relationship between the isotropic strain and density at an irradiation temperature of $1100^{\circ} \mathrm{C}$ and a fast fluence of $3.7 \times 10^{25} \mathrm{n} / \mathrm{m}^{2}$ $(\mathrm{E}>0.18 \mathrm{MeV})$.

Table 4.4: Isotropic strain at irradiation temperature of $1100^{\circ} \mathrm{C}$ and fast fluence of $3.7 \times 10^{25} \mathrm{n} / \mathrm{m}^{2}(\mathrm{E}>0.18 \mathrm{MeV})$.

\begin{tabular}{cccccccccc}
\hline$\rho\left(\mathbf{g} / \mathbf{c m}^{3}\right)$ & 1.0 & 1.2 & 1.4 & 1.5 & 1.6 & 1.8 & 1.9 & 1.96 & 2.0 \\
$f(\rho)$ & -16.15 & -13.11 & -9.98 & -8.93 & -6.97 & -4.42 & -3.41 & -2.75 & -2.33 \\
\hline
\end{tabular}

\subsubsection{PyC}

The thermal conductivity of the PyC layers is $4 \mathrm{~W} / \mathrm{m}-\mathrm{K}$ [2]. The specific heat capacity is $720 \mathrm{~J} / \mathrm{kg}-\mathrm{K}$ [7].

The radial and tangential coefficients of thermal expansion of PyC are given by [5]. The thermal expansion strains, $\varepsilon_{i}(-)$, for $i=$ radial, tangential are

$$
\varepsilon_{i}=\alpha_{i}\left(T-T_{\text {StressFree }}\right),
$$

where $\alpha_{i}$ is the thermal expansion coefficient $(1 / \mathrm{K}), T(\mathrm{~K})$ is the temperature, and $T_{\text {Stressfree }}(\mathrm{K})$ is the stress-free temperature.

Thermal expansion coefficients, $\alpha_{i}\left(10^{-6} / \mathrm{K}\right)$ for $i=$ radial, tangential are

$$
\alpha_{r}=\left(30-37.5 R_{r}\right)\left(1+0.11\left[\frac{T-673}{700}\right]\right)
$$

and

$$
\alpha_{t}=\left(36\left[R_{t}-1\right]^{2}+1\right)\left(1+0.11\left[\frac{T-673}{700}\right]\right)
$$

with

$$
R_{r}=\frac{2}{2+B A F} \quad \text { and } \quad R_{t}=\frac{1+B A F}{2+B A F}
$$

where $R_{i}(-)$ is the orientation parameter in the $i$-th direction, $T(\mathrm{~K})$ is the temperature. The Bacon anisotropy factor (BAF) is a direct measure of the crystallographic anisotropy of of pyrocarbon coatings deposited on spherical fuel particles. The BAF increases with fast fluence, and the ratio of irradiated to unirradiated BAF values is tabulated in Table 4.5 as a function of fast fluence.

Table 4.5: BAF vs. fast neutron fluence $(E>0.18 \mathrm{MeV})$.

\begin{tabular}{lcccccccc}
\hline$\phi\left(\mathbf{x 1 0} \mathbf{n}^{25} \mathbf{n} / \mathbf{m}^{2}\right)$ & 0.0 & 1.0 & 2.0 & 3.0 & 3.5 & 4.0 & 4.5 & $\geq 5.0$ \\
$\mathbf{B A F}_{\mathbf{B} A F_{\mathbf{0}}}$ & 1.0000 & 1.0019 & 1.0114 & 1.0219 & 1.0286 & 1.0324 & 1.0362 & 1.0381 \\
\hline
\end{tabular}

The Young's modulus of the PyC layers is given by $[5,6]$. The elastic modulus, $E$ (GPa), is computed as:

$$
E=\frac{E_{r}+E_{t}}{2}
$$

with its radial and tangential components, $E_{r}$ and $E_{t}$, as

$$
E_{r}=25.5\left(0.384+0.324 \times 10^{-3} \rho\right)\left(1.463-0.463 B A F_{0}\right)\left(2.985-0.0662 L_{c}\right)(1+0.23 \phi)[1+0.00015(T-20)]
$$

and

$$
E_{t}=25.5\left(0.384+0.324 \times 10^{-3} \rho\right)\left(0.481+0.519 B A F_{0}\right)\left(2.985-0.0662 L_{c}\right)(1+0.23 \phi)[1+0.00015(T-20)]
$$


where $\rho\left(\mathrm{kg} / \mathrm{m}^{3}\right)$ is the density, $B A F_{0}(-)$ is the as-fabricated BAF, $L_{c}(\AA)$ is the crystallite diameter, $\phi\left(10^{25} \mathrm{n} / \mathrm{m}^{2}\right.$, $E>0.18 \mathrm{MeV})$ is the fast neutron fluence, and $T\left({ }^{\circ} \mathrm{C}\right)$ is the temperature. The default value of $L_{c}$ is set to be $30 \AA$ such that the factor of that term takes a value of 1 . The PyC elastic modulus is plotted at different temperatures, fluences and BAFs in Figure 4.3.

Poisson's ratio is set to a constant value of $0.33[5,6]$.

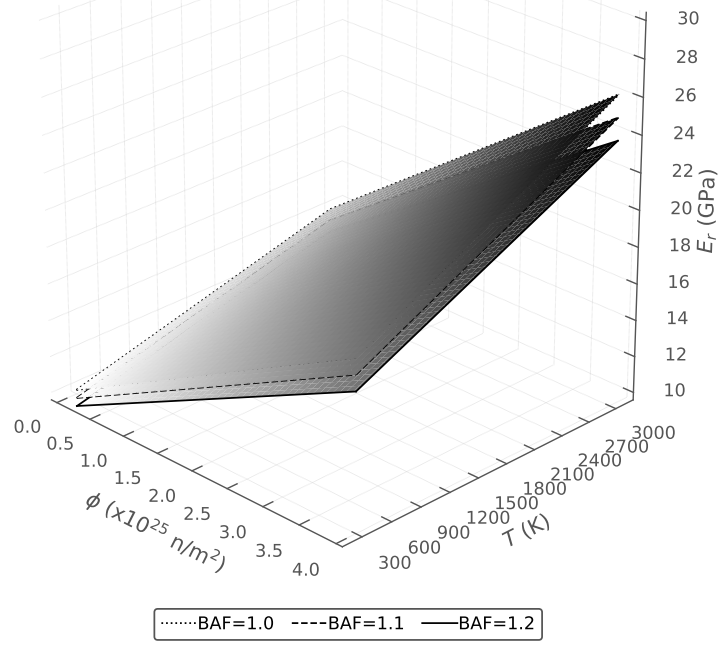

(a) Radial.

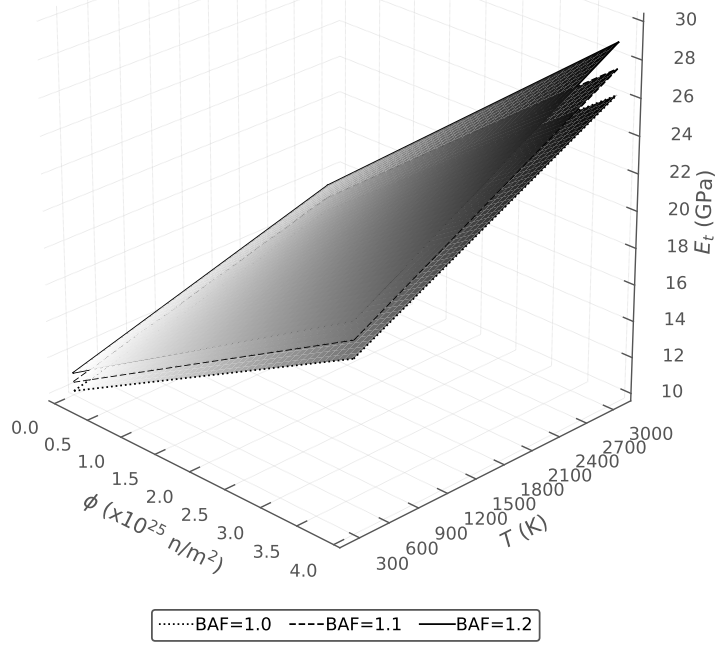

(b) Tangential.

Figure 4.3: The PyC elastic modulus.

The irradiation creep model for the $\mathrm{PyC}$ is the same as for the buffer. Poisson's ratio in creep of the PyC layers is set to a constant value of 0.5 .

Under irradiation, pyrolytic carbon shrinks in both the radial and tangential directions. At modest fast neutron fluences, however, it begins to swell in the radial direction. The anisotropic strains, $\varepsilon_{i}(\%)$ for $i=$ radial, tangential,isotropic are given by $[5,6]$ :

$$
\varepsilon_{i}=a_{1} \phi+a_{2} \phi^{2}+a_{3} \phi^{3}+a_{4} \phi^{4}
$$

where $\phi\left(10^{25} \mathrm{n} / \mathrm{m}^{2}, E>0.18 \mathrm{MeV}\right)$ is the fast neutron fluence, and $a_{1}$ through $a_{4}$ are BAF- and temperature-dependent polynomial coefficients $\left(\rho_{0}=1.96 \mathrm{~g} / \mathrm{cm}^{3}\right)$ at varied temperatures.

The PyC radial and tangential strain with their polynomial coefficients are plotted in Figure 4.4 and Figure 4.5, respectively. The polynomial coefficients at other BAF are interpolated or extrapolated from these tables while at other temperature they are only interpolated from these tables. Adjustments for other density are made by applying an anisotropic scaling factor, $v$. The anisotropic scaling factor is defined as the ratio of the difference between the radial and tangential strain components at a given density to the difference between the radial and tangential strain components at $\rho_{0}$ :

$$
v=\frac{\varepsilon_{r}(\rho)-\varepsilon_{t}(\rho)}{\varepsilon_{r}\left(\rho_{0}\right)-\varepsilon_{t}\left(\rho_{0}\right)} .
$$

The anisotropic scaling factor is calculated using the values in Table 4.6, which shows the relationship between the difference between the radial and tangential strain components and density at an irradiation temperature and a fast fluence. Thus, the adjusted radial and tangential strains become

$$
\begin{aligned}
& \varepsilon_{r}(\rho)=\mu \varepsilon_{i s o}\left(\rho_{0}\right)+v\left[\varepsilon_{r}\left(\rho_{0}\right)-\varepsilon_{i s o}\left(\rho_{0}\right)\right], \\
& \varepsilon_{t}(\rho)=\mu \varepsilon_{i s o}\left(\rho_{0}\right)+v\left[\varepsilon_{t}\left(\rho_{0}\right)-\varepsilon_{i s o}\left(\rho_{0}\right)\right] .
\end{aligned}
$$




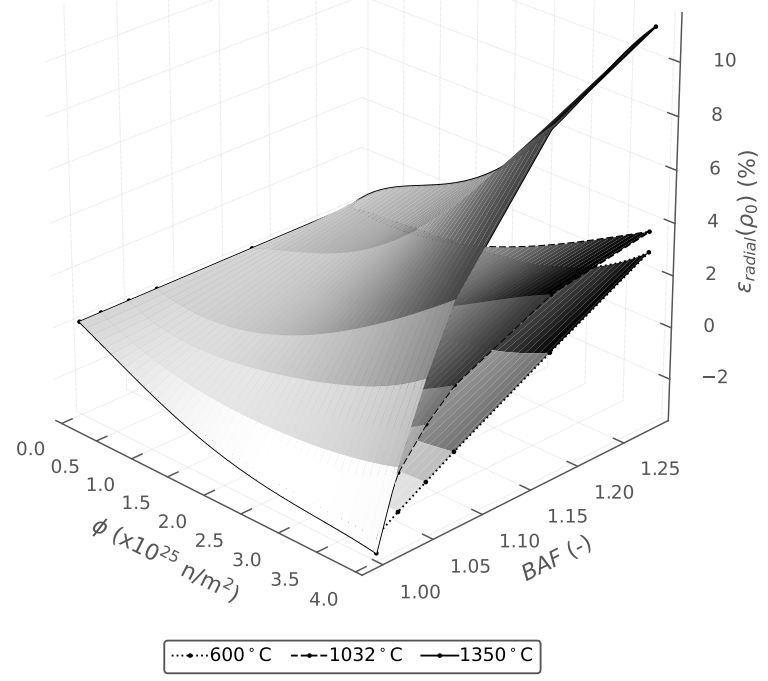

Figure 4.4: The PyC radial strain at $\rho_{0}=1.96 \mathrm{~g} / \mathrm{cm}^{3}$. Polynomial coefficients for radial strain components are tabulated below:

\begin{tabular}{cccccccc}
\hline & $\mathbf{B A F}=$ & $\mathbf{1 . 0 0 0 0}$ & $\mathbf{1 . 0 2 1 2}$ & $\mathbf{1 . 0 4 8 8}$ & $\mathbf{1 . 0 7 6 9}$ & $\mathbf{1 . 1 7 4 6}$ & $\mathbf{1 . 2 7 8 7}$ \\
\hline $600^{\circ} \mathrm{C}$ & $a_{1}$ & -1.24080 & -1.10640 & -0.94333 & -0.78045 & -0.15714 & 0.40265 \\
& $a_{2}$ & 0.00175 & -0.03128 & -0.03589 & -0.02975 & -0.14889 & -0.16501 \\
& $a_{3}$ & 0.08533 & 0.09184 & 0.08184 & 0.06655 & 0.07546 & 0.03676 \\
& $a_{4}$ & -0.01253 & -0.01220 & -0.00958 & -0.00626 & -0.00293 & 0.00706 \\
\hline $1032^{\circ} \mathrm{C}$ & $a_{1}$ & -1.52390 & -2.07520 & -2.00470 & -1.81690 & -1.18540 & -0.45900 \\
& $a_{2}$ & 0.13048 & 1.37845 & 1.30380 & 1.10850 & 0.64995 & 0.51172 \\
& $a_{3}$ & 0.06299 & -0.48993 & -0.37280 & -0.23868 & 0.01380 & -0.03245 \\
& $a_{4}$ & -0.01072 & 0.06602 & 0.04538 & 0.02484 & -0.01284 & -0.00142 \\
\hline $1350^{\circ} \mathrm{C}$ & $a_{1}$ & -1.42840 & -1.54330 & -1.49640 & -0.89522 & 1.20930 & 3.71620 \\
& $a_{2}$ & -0.19563 & 0.59804 & 1.16621 & 0.80331 & -0.53861 & -2.70420 \\
& $a_{3}$ & 0.18991 & -0.09997 & -0.30106 & -0.09009 & 0.43114 & 1.17990 \\
& $a_{4}$ & -0.02591 & 0.00978 & 0.03475 & 0.00467 & -0.05590 & -0.13910 \\
\hline
\end{tabular}

Table 4.6: Isotropic strain at irradiation temperature of $1100^{\circ} \mathrm{C}$ and fast fluence of $3.7 \times 10^{25} \mathrm{n} / \mathrm{m}^{2}(E>0.18 \mathrm{MeV})$.

\begin{tabular}{lccccccccc}
\hline$\rho\left(\mathbf{g} / \mathbf{c m}^{\mathbf{3}}\right)$ & 1.00 & 1.20 & 1.40 & 1.50 & 1.60 & 1.80 & 1.90 & 1.96 & 2.00 \\
$\varepsilon_{\text {iso }}(\boldsymbol{\%})$ & -16.15 & -13.11 & -9.98 & -8.93 & -6.97 & -4.42 & -3.41 & -2.75 & -2.33 \\
$\varepsilon_{\mathbf{r}}-\varepsilon_{\mathbf{t}}(\boldsymbol{\%})$ & 0.000 & 0.500 & 1.100 & 1.650 & 2.450 & 6.305 & 7.900 & 9.600 & 11.100 \\
\hline
\end{tabular}




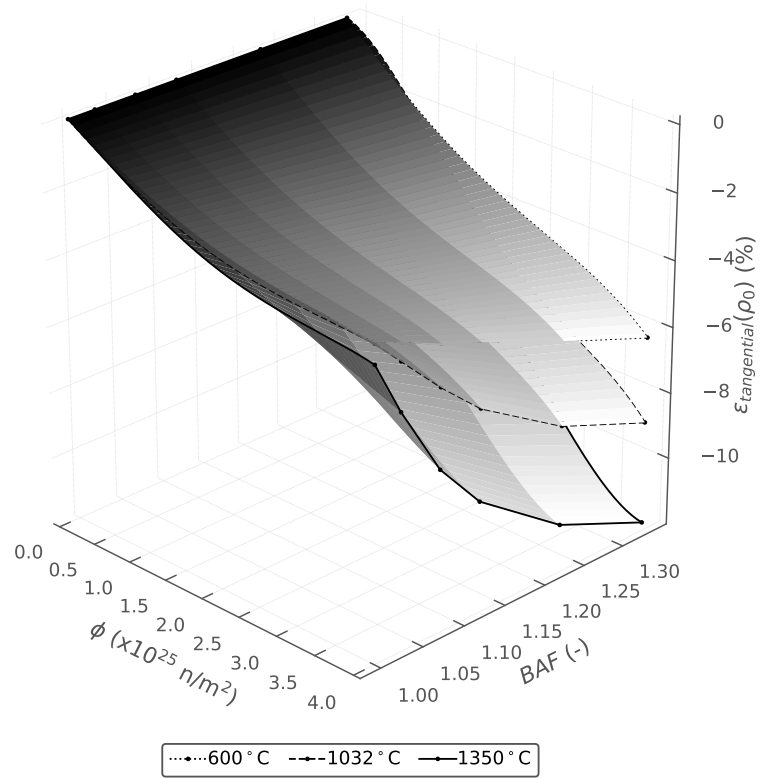

Figure 4.5: The PyC tangential strain at $\rho_{0}=1.96 \mathrm{~g} / \mathrm{cm}^{3}$. Polynomial coefficients for tangential strain components are tabulated below:

\begin{tabular}{cccccccc}
\hline & $\mathbf{B A F}=$ & $\mathbf{1 . 0 0 0 0}$ & $\mathbf{1 . 0 3 0 3}$ & $\mathbf{1 . 0 7 6 9}$ & $\mathbf{1 . 1 2 5 0}$ & $\mathbf{1 . 2 2 5 8}$ & $\mathbf{1 . 3 3 3 3}$ \\
\hline $600^{\circ} \mathrm{C}$ & $a_{1}$ & -1.24080 & -1.38550 & -1.46790 & -1.64660 & -1.84990 & -2.19190 \\
& $a_{2}$ & 0.00175 & 0.05307 & -0.02836 & 0.03928 & -0.09358 & 0.02675 \\
& $a_{3}$ & 0.08533 & 0.07620 & 0.12139 & 0.10067 & 0.18119 & 0.15352 \\
& $a_{4}$ & -0.01253 & -0.01245 & -0.01948 & -0.01764 & -0.03036 & -0.02972 \\
\hline $1032^{\circ} \mathrm{C}$ & $a_{1}$ & -1.52390 & -1.57590 & -1.32200 & -1.18700 & -0.96963 & -0.81239 \\
& $a_{2}$ & 0.13048 & 0.09019 & -0.51928 & -0.90635 & -1.59110 & -2.20760 \\
& $a_{3}$ & 0.06299 & 0.05306 & 0.27603 & 0.41046 & 0.64689 & 0.88496 \\
& $a_{4}$ & -0.01072 & -0.00815 & -0.03465 & -0.05067 & -0.07682 & -0.10457 \\
\hline $1350^{\circ} \mathrm{C}$ & $a_{1}$ & -1.42840 & -2.24680 & -2.82930 & -3.25550 & -4.44780 & -5.67140 \\
& $a_{2}$ & -0.19563 & 0.48243 & 0.76088 & 0.90423 & 1.60320 & 2.41920 \\
& $a_{3}$ & 0.18991 & -0.07687 & -0.22314 & -0.33175 & -0.58683 & -0.86155 \\
& $a_{4}$ & -0.02591 & 0.00464 & 0.02431 & 0.04329 & 0.07458 & 0.10668 \\
\hline
\end{tabular}




\subsection{4 $\mathrm{SiC}$}

The thermal conductivity model is given by Miller [5].

$$
k=\frac{17885}{T_{K}}+2.0
$$

where $k(\mathrm{~W} / \mathrm{m}-\mathrm{K})$ is the thermal conductivity and $T_{K}(\mathrm{~K})$ is the temperature.

The correlation for specific heat capacity, $c_{P}(\mathrm{~J} / \mathrm{kg}-\mathrm{K})$, is given by [11].

$$
c_{P}=925.65+0.3772 T-7.9259 \times 10^{-5} T^{2}-\frac{3.1946 \times 10^{7}}{T^{2}}
$$

where $T(\mathrm{~K})$ is the temperature.

The thermal expansion coefficient is $4.9 \times 10^{-6}(1 / \mathrm{K})[5,6]$.

The Young's modulus and Poisson's ratio of $\mathrm{SiC}$ is given by [5].

The Young's modulus (E) is temperature-dependent and given according to Table 4.7. For values between the tabulated values, linear interpolation is used. For temperatures below $25.0^{\circ} \mathrm{C}$ Young's modulus is taken as $428.0 \mathrm{GPa}$. For temperatures above $1600^{\circ} \mathrm{C}$, a value of $198.0 \mathrm{GPa}$ is used.

Table 4.7: Elastic modulus of the $\mathrm{SiC}$ as a function of temperature.

\begin{tabular}{lcccc}
\hline $\mathbf{T}\left({ }^{\circ} \mathbf{C}\right)$ & 25.0 & 940.0 & 1215.0 & 1600.0 \\
$\mathbf{E}(\mathbf{G P a})$ & 428.0 & 375.0 & 340.0 & 198.0 \\
\hline
\end{tabular}

Poisson's ratio is set to a constant value of 0.13 .

\subsection{Fission gas release model}

\subsubsection{Fission gas}

The release of long-lived fission gases from the kernel is modeled as a two-step process: first, the gas atoms are driven through the grain towards the grain boundary; second, the gas atoms migrate from the grain boundary to the free surface of the fuel where they are instantaneously released into the free volume of the TRISO particle. The model includes both direct recoil and diffusion to grain boundaries. The release fraction, $F G R(-)$, which corresponds to the fraction of the gas generated by fission that is released by the kernel is given by:

$$
F G R=\left(R F_{\text {recoil }}+\left[1.0-R F_{\text {recoil }}\right] R F_{\text {Booth }}\right) F G P
$$

where $R F_{\text {recoil }}(-)$ and $R F_{\text {Booth }}(-)$ are the release fractions of fission gas by direct recoil and diffusion, respectively, and $F G P(\mathrm{~mol})$ is the amount of fission gas produced in the kernel. The model assumes $100 \%$ of the fission gas is released to the void volume.

\subsubsection{Direct recoil}

Direct kinetic release of fission gases from the kernel to the buffer is accounted for by geometrical considerations and fission fragment ranges derived from compiled experimental data [12]. The fission gas mixture is essentially composed of krypton and xenon, with relative fractions of 18.5 and $81.5 \%$ [5]. Hence, the recoil fraction is given by:

$$
R F_{\text {recoil }}=0.185 R F_{\text {recoil }, K r}+0.815 R F_{\text {recoil }, X e}
$$

where $R F_{\text {recoil }, K r}(-)$ and $R F_{\text {recoil }, X e}(-)$ are the respective release fractions of krypton and xenon due to recoil. They depend on the ranges of krypton and xenon in $\mathrm{UCO}$ (i.e., $r_{i}$ for $\left.i=\mathrm{Kr}, \mathrm{Xe}\right)$ and on the radius of the kernel, $r_{k}(\mathrm{~m})$ :

$$
R F_{\text {recoil }, i}=0.25\left(\frac{r_{k}^{3}-\left[r_{k}-r_{i}\right]^{3}}{r_{k}^{3}}\right) .
$$


The ranges of krypton and xenon in $\mathrm{UCO}$ (i.e., $r_{i}$ for $i=\mathrm{Kr}, \mathrm{Xe}$ ) are obtained from their individual ranges in uranium, carbon, and oxygen:

$$
r_{i}=10.0\left(\frac{r_{i, U}+O U r_{i, O}+C U r_{i, C}}{\rho_{k}[1.0+O U+C U]}\right)
$$

where $O U(-)$ and $C U(-)$ are the initial oxygen to uranium and carbon to uranium ratios, $\rho_{k}\left(\mathrm{~g} / \mathrm{cm}^{3}\right)$ is the density of the kernel, and the individual ranges are given in Table 4.8.

Table 4.8: Range of $\mathrm{Kr}$ and $\mathrm{Xe}$ in $\mathrm{U}, \mathrm{C}$, and $\mathrm{O}$ [12].

\begin{tabular}{lcccccc}
\hline Fission product & $\mathrm{Kr}$ & $\mathrm{Kr}$ & $\mathrm{Kr}$ & $\mathrm{Xe}$ & $\mathrm{Xe}$ & $\mathrm{Xe}$ \\
Medium & uranium & carbon & $\begin{array}{c}\mathrm{X} \text { oxygen } \\
\text { uranium }\end{array}$ & $\begin{array}{c}\text { carbon } \\
\text { oxygen }\end{array}$ \\
Range $\left(\mathbf{m g} / \mathbf{c m}^{\mathbf{2}}\right)$ & 11.7 & 3.3 & 3.1 & 8.3 & 2.3 & 2.2 \\
\hline
\end{tabular}

\subsubsection{Booth model}

Diffusive release through kernel grains to the grain boundaries and subsequent transport through the interconnected porosity is estimated by the Booth equivalent sphere diffusion model [13]. The Booth release fraction is given by:

$$
R F_{\text {Booth }}=1.0-\frac{6.0 r_{\text {grain }}^{2}}{D t} \sum_{n=1}^{\infty} \frac{1.0-\exp \left(\frac{-n^{2} \pi^{2} D t}{r_{\text {grain }}^{2}}\right)}{n^{4} \pi^{4}}
$$

where $r_{\text {grain }}(\mathrm{m})$ is the radius of the diffusing sphere (i.e., the average grain radius), $D\left(\mathrm{~m}^{2} / \mathrm{s}\right)$ is the diffusivity of the fission gas in the grain, and $t(\mathrm{~s})$ is the diffusion time.

The UCO model utilizes an effective diffusion coefficient, $D_{\text {eff }}\left(\mathrm{m}^{2} / \mathrm{s}\right)$, formulated for $\mathrm{UO}_{2}$ fuel by [14] as:

$$
D_{\text {eff }}=\sum_{i=1}^{3} D_{i}=D_{1}+D_{2}+D_{3}
$$

where $D_{1}, D_{2}$, and $D_{3}$ are the diffusion coefficients of the mechanisms controlling diffusion at different temperature ranges.

- At the highest temperatures, diffusion proceeds through cation lattice by means of thermally activated vacancies:

$$
D_{1}=7.6 \times 10^{-10} \exp \left(-\frac{35225}{T_{K}}\right)
$$

where $T_{K}(\mathrm{~K})$ is the temperature of the kernel.

- At intermediate temperatures, diffusion is driven by means of vacancies produced by the irradiation process:

$$
D_{2}=s^{2} j_{v}\left(\frac{K^{\prime}}{Z j_{v}}\right)^{0.5}
$$

where $s(\mathrm{~m})$ is the atomic jump distance, $j_{v}\left(s^{-1}\right)$ is the cation vacancy jump rate, $K^{\prime}\left(s^{-1}\right)$ is the rate of defect production per atom, and $\mathrm{Z}(-)$ is the number of sites around a point defect from which recombination is inevitable. They are given by:

$$
\begin{gathered}
s=3.0 \times 10^{-10}, \\
j_{v}=10^{13} \exp \left(-\frac{27778}{T_{K}}\right), \\
K^{\prime}=\frac{K B_{u}}{t},
\end{gathered}
$$


and

$$
Z=2
$$

where $\mathrm{K}\left(10^{4}\right.$ defects/fission $)$ is the damage rate and $B_{u}$ (\%FIMA) is the burnup.

Finally, at lower temperatures, a term proportional to the fission rate density, $f^{\prime \prime \prime}$ (fission $/ \mathrm{m}^{3}$-s), is used to agree with experimental results reported by [14]:

$$
D_{3}=2.0 \times 10^{-40} f^{\prime \prime \prime}
$$

\subsubsection{Fission gas production}

The amount of fission gas produced by the kernel, $F G P(\mathrm{~mol})$, during a time interval $\Delta t(\mathrm{~s})$, is calculated as:

$$
F G P=\frac{\Gamma_{F G} f^{\prime \prime \prime} V_{k} \Delta t}{N_{\text {avo }}}
$$

where $\Gamma_{F G}(-)$ is the combined fractional fission yield of krypton and xenon atoms per fission, $V_{k}\left(\mathrm{~m}^{3}\right)$ is the volume of the kernel, and $N_{\text {avo }}\left(6.022 \times 10^{23} \mathrm{at} / \mathrm{mol}\right)$ is Avogadro's number.

\subsubsection{Fission yields}

Fission yields, $\Gamma$ (atoms/fission), for silver, cesium, strontium, and krypton and xenon combined are given below.

- For silver:

$$
\Gamma_{A g}= \begin{cases}1.31625 \times 10^{-3} b^{0.55734} & \text { for } e<17.5 \\ 8.24492 \times 10^{-4} b^{0.53853} & \text { for } e \geq 17.5\end{cases}
$$

with $b=\max (1.0, B u)$.

- For cesium:

$$
\Gamma_{C s}= \begin{cases}0.14 & \text { for } e<17.5 \\ 0.16 & \text { for } e \geq 17.5\end{cases}
$$

- For strontium:

$$
\Gamma_{S r}= \begin{cases}0.11754 b^{-0.21762} & \text { for } e<17.5 \\ 0.11819 b^{-0.15778} & \text { for } e \geq 17.5\end{cases}
$$

with $b=\max (0.6, B u)$.

- For krypton and xenon:

$$
\Gamma_{K r+X e}=0.297
$$

where $B_{u}$ (\%FIMA) is the burnup and $e(\%)$ is the ${ }^{235} \mathrm{U}$ enrichment [5].

\subsubsection{Diffussion coefficients calculated by lower length scale modeling}

The effective diffusion coefficients $D_{e f f}$ were derived from experimental data on $\mathrm{UO}_{2}$ due to lack of relevant UCO data. To overcome this limitation, lower length scaling modeling is performed by LANL to compute diffusion coefficients specifically for UCO kernel. The fitted $D_{1}$ and $D_{2}$ terms from their simulation are given as

$$
D_{1}=5.2111 \times 10^{-2} \exp \left(-79948 / T_{k}\right)+3.4834 \times 10^{-9} \exp \left(-51197 / T_{k}\right)
$$

and

$$
D_{2}=5.086 \times 10^{-7} \exp \left(-50859 / T_{k}\right)
$$

The comparison of old and new effective diffusivities at different temperatures is plotted in Figure 4.6. Below 1000 $\mathrm{K}, D_{3}$ is dominated so the new and old diffusivity are the same. Between $1000 \mathrm{~K}$ and $2500 \mathrm{~K}$, the new diffusivity for $\mathrm{UCO}$ kernel is lower than the old one for $\mathrm{UO}_{2}$ kernel, because the presence of UC phase slows down the Xe diffusion. Their difference reaches maximum near the temperature of $1400 \mathrm{~K}$. 


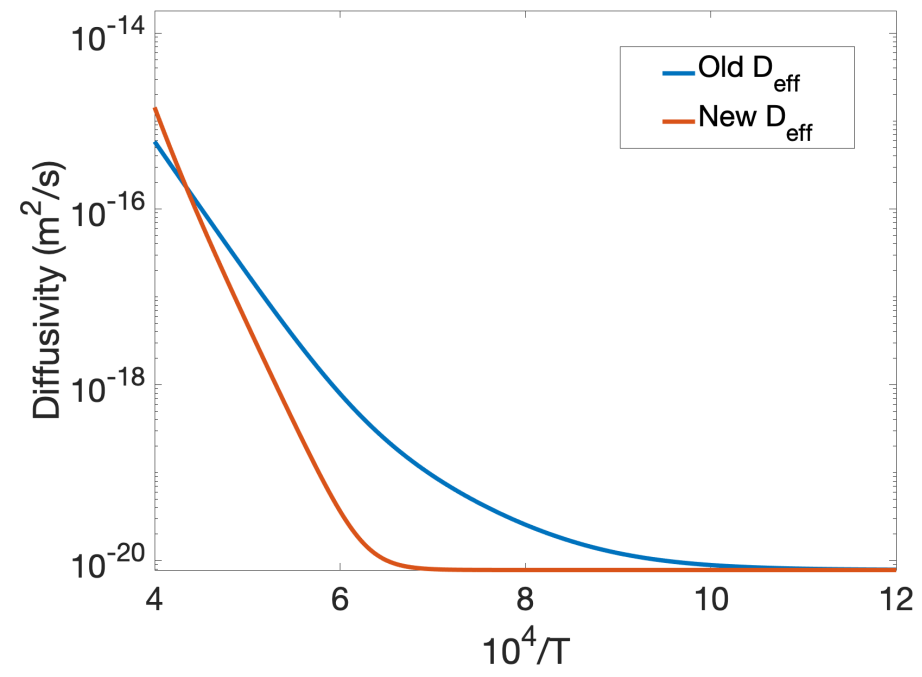

Figure 4.6: Comparision of new and old effective diffusivity.

\subsection{Failure analysis}

The ability of the fuel to contain fission products is largely dictated by the quality of the manufacturing process because most of the fission product release is expected to occur due to failure of coating layers a small number of particles at the locations of defects. To account for statistical variation in physical dimensions and material properties from particle to particle, a Monte Carlo scheme is utilized to compute failure probability for a statistically sampled batch of particles. This enables realistic calculations of fission product release from the many particles in a TRISO-fueled reactor.

\subsubsection{Failure modes}

There are several potential failure mechanisms for TRISO fuel, outlined in the sections below, that are considered in current work. The implementation of other failure modes is under active development.

\subsubsection{Pressure Vessel Failure}

Early during irradiation, pyrocarbon layers shrink and put the SiC layer in compression. As irradiation progresses, the creep of the pyrocarbon layers tends to relax some of this compressive stress. In addition, the buildup of fission gas pressure tends to put the coating layers in tension. Figure 4.7 shows the tangential stress at the inner surface of the SiC layer under three temperature conditions. This stress value is important as it is used to determine whether a particle fails. Since CO production in UCO kernel is relatively small, gas pressure is usually not high enough to make the tangential stress in the $\mathrm{SiC}$ layer become tensile. The pressure vessel failure is not likely to occur for an intact TRISO UCO kernel.

\subsubsection{Cracking of IPyC}

During irradiation, shrinkage of the pyrocarbon layers causes a significant tensile stress in those layers. If the stress exceeds the tensile strength of that material, a radial crack forms in that pyrocarbon layer. The radial crack leads to a high local tensile stress in the $\mathrm{SiC}$ layer adjacent to that cracked pyrocarbon layer that could lead to particle failure. As shown in Figure 4.8a and Figure 4.8b, the stress in the SiC layer near the crack tip is elevated significantly due to the development of cracking in the IPyC layer. 


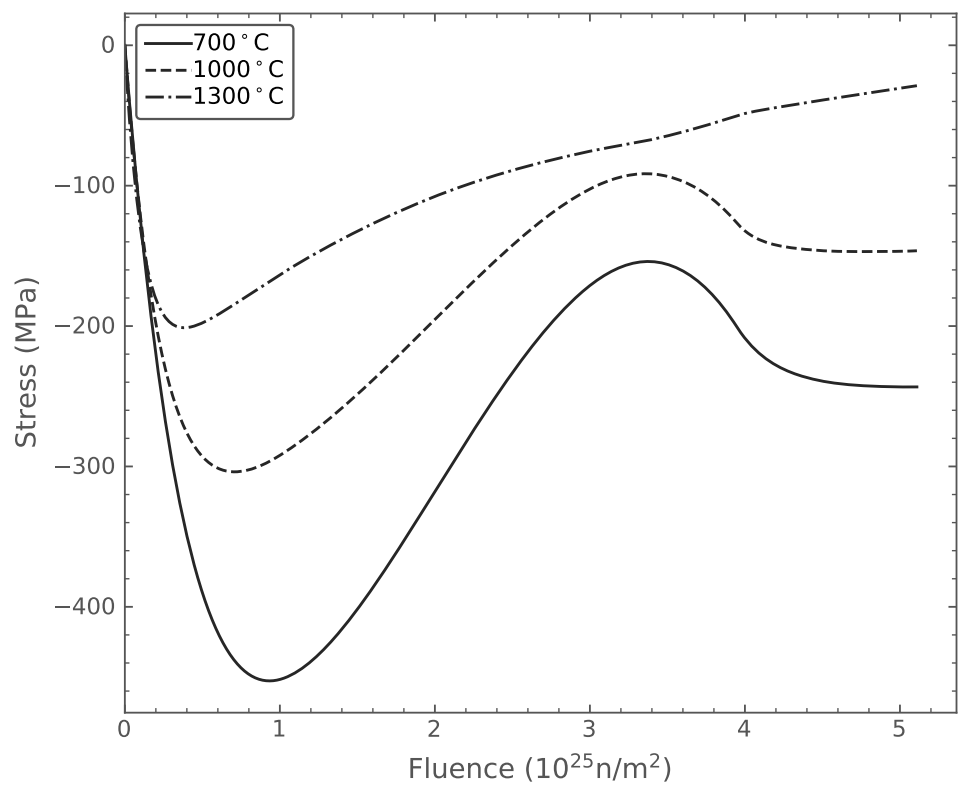

Figure 4.7: Tangential stress at the inner surface of the $\mathrm{SiC}$ layer.

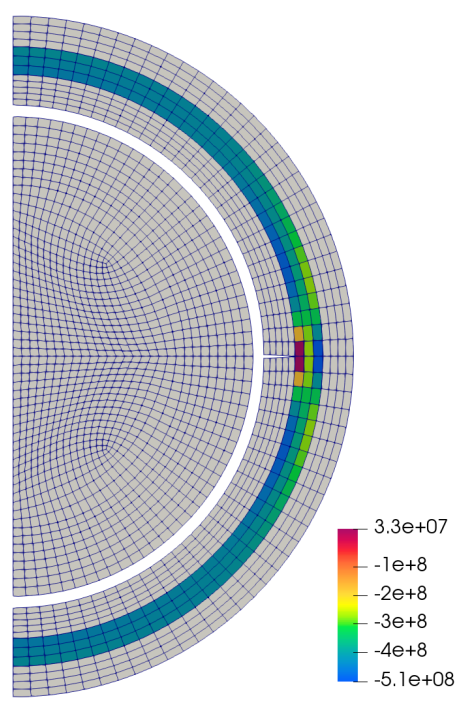

(a) Stress contour for $\mathrm{T}=700^{\circ} \mathrm{C}$ when maximum tangential stress is reached.

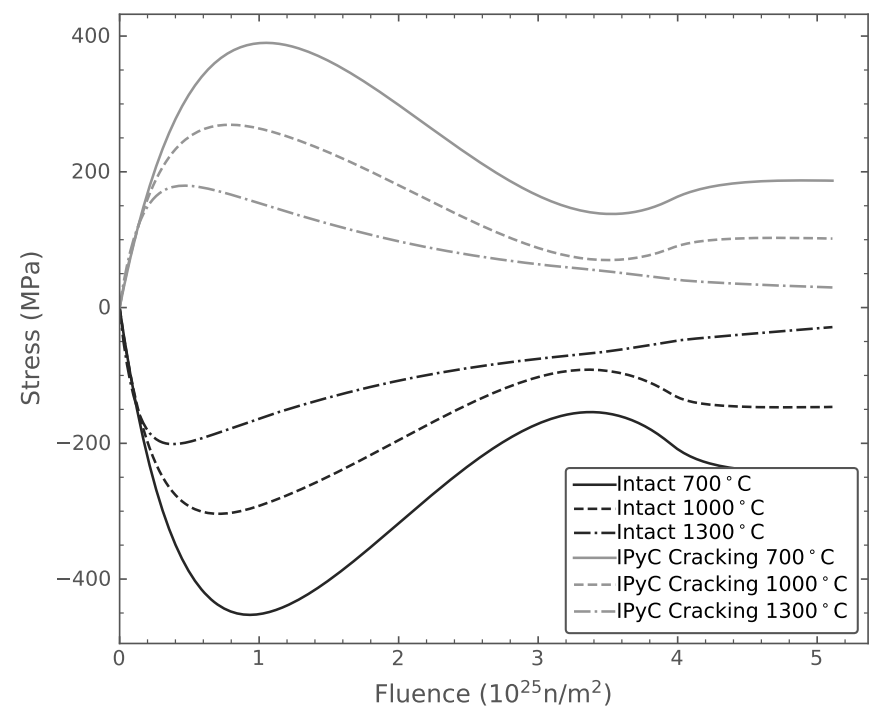

(b) Inner wall tangential stress for $\mathrm{SiC}$.

Figure 4.8: Cracking of IPyC.

\subsubsection{Pressure vessel failure of an aspherical particle}

A single flat facet on one side of the particle created during fabrication is a common cause of aspherical behavior in particle fuel. The degree of asphericity for a particle is measured by the aspect ratio. During irradiation, the faceted portion of the particle acts as a flat plate that retains the internal gas pressure. If the pressure builds up high enough, this results in a local region of tensile stress in the central portion of the plate, which can contribute to particle failures. As shown in Figure $4.9 \mathrm{~b}$, the tangential stresses in $\mathrm{SiC}$ layer remain compressive through end of irradiation because the $\mathrm{CO}$ production is limited in UCO fuel. 


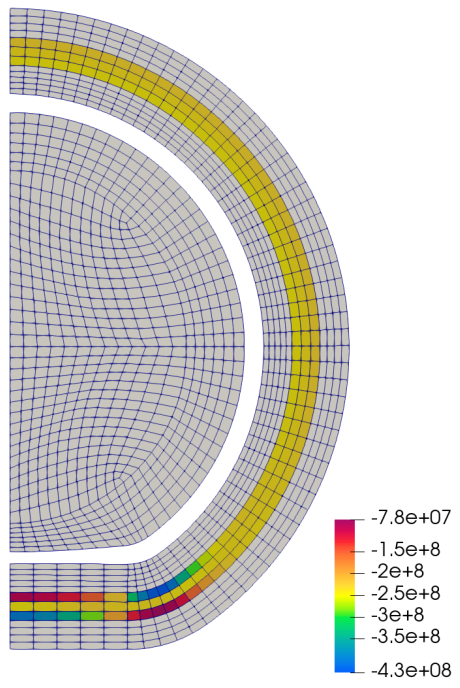

(a) Stress contour for $\mathrm{T}=700^{\circ} \mathrm{C}$ at end of irradiation with an aspect ratio of 1.04 .

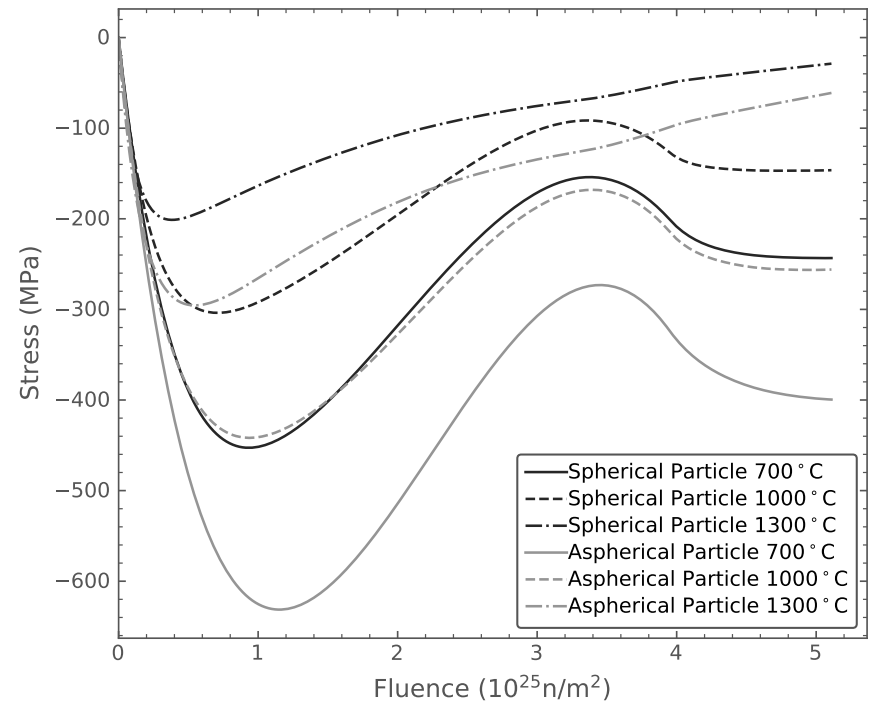

(b) Stress in the faceted portion for SiC.

Figure 4.9: Aspherical Particle.

\subsubsection{Weibull failure theory}

A Weibull failure criterion is used to determine vessel failure for the inner pyrolytic carbon (IPyC) layer, silicon carbide $(\mathrm{SiC})$ layer, and outer pyrolytic carbon $(\mathrm{OPyC})$ layer. The maximum stress $\sigma_{c}$ is compared to a strength that is sampled from a Weibull distribution having mean strength $\sigma_{m s}$ to determine whether or not failure occurs. The cumulative distribution function for the Weibull distribution is given as:

$$
P=1-\exp \left(-\left(\frac{\sigma_{c}}{\sigma_{m s}}\right)^{m}\right)
$$

The effective mean strength $\sigma_{m s}$ is given as:

$$
\sigma_{m s}=\frac{\sigma_{0}}{I_{n}^{\frac{1}{m}}}
$$

where $\sigma_{0}$ is the characteristic strength, $m$ is the Weibull modulus. The integral $I_{n}$ is a normalized integration of the stress distribution using the principle of independent action (PIA) model as follows:

$$
I_{n}=\frac{\int_{V}\left(\sigma_{1}^{m}+\sigma_{2}^{m}+\sigma_{3}^{m}\right) d V}{\sigma_{c}^{m}}
$$

where $\sigma_{c}$ is the maximum value calculated for a principal stress anywhere in the volume and $\sigma_{1}, \sigma_{2}$, and $\sigma_{3}$ are three principal stresses. The negative principal stresses are not included in this integral because the compressive stresses do not contribute to fracture.

The Weibull modulus $(m)$ and characteristic strength $\left(\sigma_{0}\right)$ for the $\mathrm{SiC}$ are held constant throughout irradiation and are given as:

$$
m=6 \text { and } \sigma_{0}=9640000 \mathrm{~Pa}-\mathrm{m}^{3 / 6}
$$

The Weibull modulus for the PyC is assumed to be 9.5 , which corresponds to a density of $1.9\left(\mathrm{~g} / \mathrm{cm}^{3}\right)$.

The Weibull characteristic strength of the $\mathrm{PyC}[5,6]$ is a function of anisotropy and is determined from the following equation for room temperature:

$$
\sigma_{0}=10^{6} \times\left(154.46 X^{2}-141.1 X\right)
$$

where $X$ is a fitting parameter with a default value of 1.02 . The characteristic strength has units of Pa- $\mathrm{m}^{3 / 9.5}$ when the Weilbull modulus is 9.5 . 
At other temperatures and nonzero fluence, Equation 4.57 is multiplied by a factor as follows:

$$
\text { factor }=[(1+0.23 \phi)(1+0.00015 T)]^{1 / 2}
$$

where $\phi\left(10^{25} \mathrm{n} / \mathrm{m}^{2}, \mathrm{E}>0.18 \mathrm{MeV}\right)$ is the fast neutron fluenc and $\mathrm{T}\left({ }^{\circ} \mathrm{C}\right)$ is the temperature.

\subsubsection{High-fidelity analysis}

For computational efficiency, it is important that the fuel performance calculations are primarily conducted using 1D models because they are run within a Monte Carlo sampling procedure. However, the stress concentrations due to the presence of phenomena such as cracking must be characterized using a higher-dimensionality, higher-fidelity model. To account for these multi-dimensional phenomena within a 1D fuel performance model, a high-fidelity analysis is first performed to obtain the mean strength based on the multi-dimensional stress distribution and stress correlation factor. The multi-dimensional strength and stress correlation factors are calculated prior to statistical modeling and then used in the 1D model to determine failure.

\subsubsection{Stress correlation for IPyC cracking}

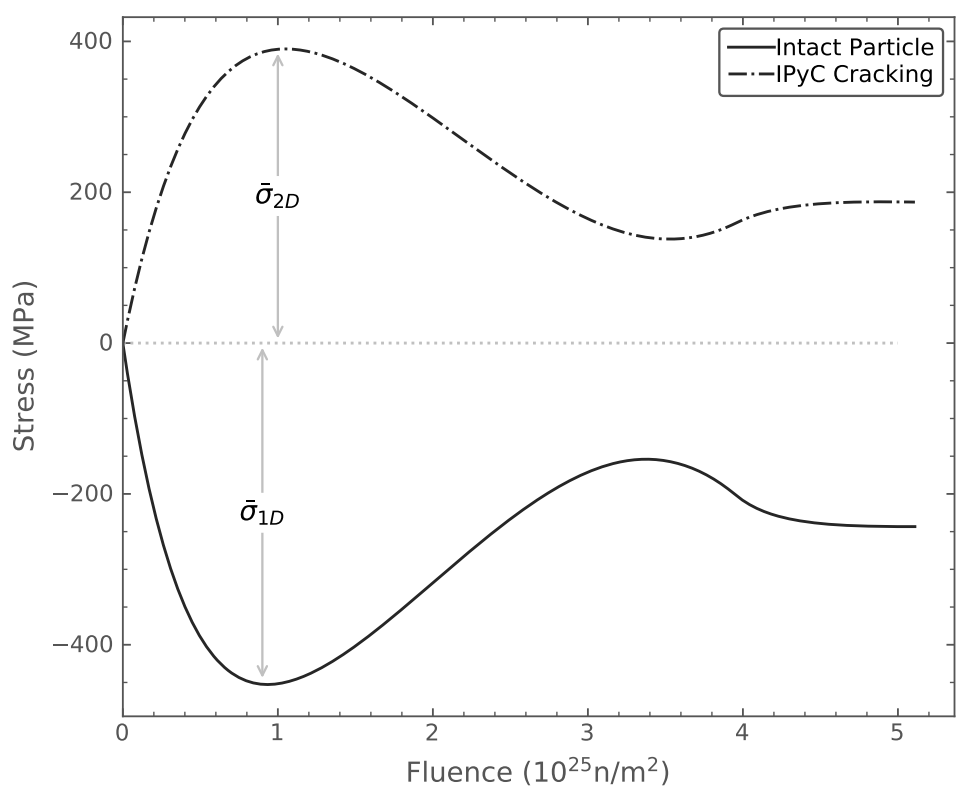

Figure 4.10: Stress correlation for IPyC cracking.

The maximum tangential stress histories in SiC layer for a cracked particle (near crack tip) and intact particle are shown in Figure 4.10. The maximum SiC stress in a cracked particle is approximated as

$$
\sigma_{\text {correlation }}=\frac{\bar{\sigma}_{2 \mathrm{D}}}{\bar{\sigma}_{1 \mathrm{D}}} \sigma_{1 \mathrm{D}}
$$

where $\bar{\sigma}_{2 \mathrm{D}}$ and $\bar{\sigma}_{1 \mathrm{D}}$ are the maximum stress calculated in the $2 \mathrm{D}$ and $1 \mathrm{D}$ analysis at the mean values for a specified batch of particles, respectively. Upon varying statistical parameters, the maximum stress in the SiC layer is determined from the one dimensional finite element solution for $\sigma_{1 \mathrm{D}}$. The mean strength of the $2 \mathrm{D}$ model that is evaluated at maximum tangential stress state will be used for 1D analysis.

\subsubsection{Stress correlation for aspherical particle}

The tangential stress histories for a representative faceted and spherical fuel particles are shown in Figure 4.11. In evaluating asphericity, a second term is added to correctly estimate the maximum stress $\sigma_{c}$ for an aspherical particle: 


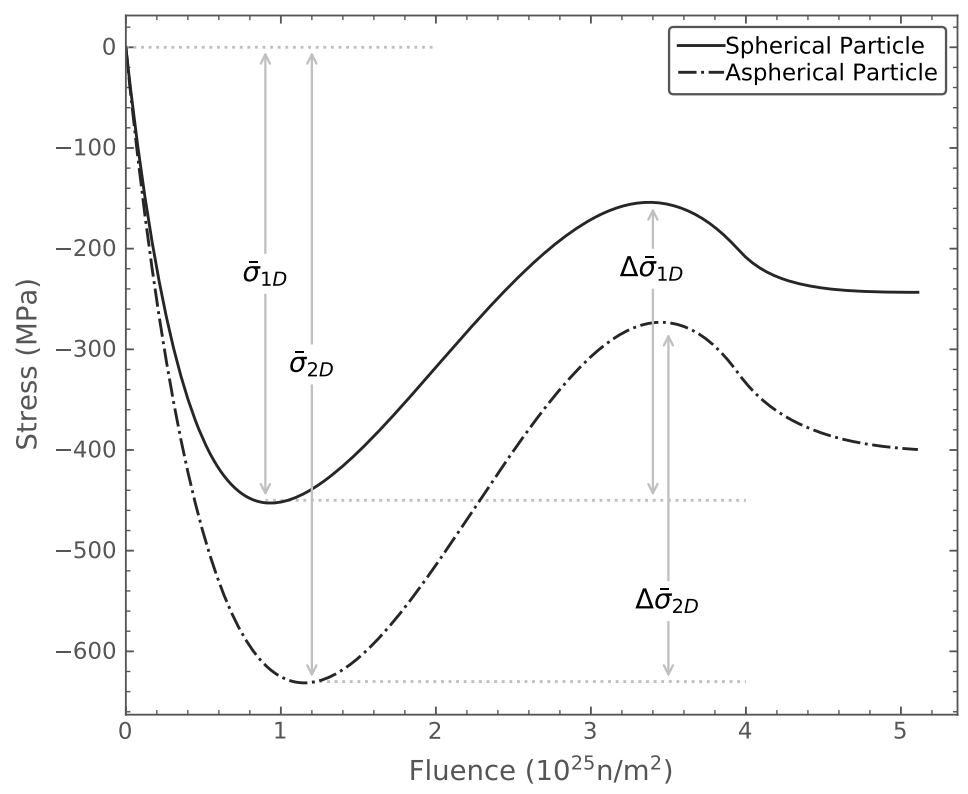

Figure 4.11: Stress correlation for aspherical particle.

$$
\sigma_{\text {correlation }}=\frac{\bar{\sigma}_{2 \mathrm{D}}}{\bar{\sigma}_{1 \mathrm{D}}} \sigma_{1 \mathrm{D}-\min }+\frac{\Delta \bar{\sigma}_{2 \mathrm{D}}}{\Delta \bar{\sigma}_{1 \mathrm{D}}} \Delta \sigma_{1 \mathrm{D}}
$$

where $\Delta \bar{\sigma}_{2 \mathrm{D}}, \Delta \bar{\sigma}_{1 \mathrm{D}}$ and $\Delta \sigma_{1 \mathrm{D} \text {-min }}$ are changes in the stresses $\bar{\sigma}_{2 \mathrm{D}}, \bar{\sigma}_{1 \mathrm{D}}$ and $\sigma_{1 \mathrm{D}-\mathrm{min}}$ going from the minimum to the end of irradiation. If a second extremum (or maximum) occurs before the end of irradiation, then $\Delta \bar{\sigma}_{2 \mathrm{D}}, \Delta \bar{\sigma}_{1 \mathrm{D}}$ and $\Delta \sigma_{1 \mathrm{D}}$ are taken as changes in these stress going from the minimum to the maximum. The additional term is needed for asphericity evaluations because failures due to asphericity occur after the first extremum for $\sigma_{1 \mathrm{D}}$ has been reached, when shrinkage effects from the $\mathrm{PyC}$ are diminishing and inner pressure keeps accumulating. The mean strength of the 2D model that is evaluated at the end of irradiation will be used for 1D analysis.

\subsubsection{Monte Carlo Scheme}

Figure 4.12 depicts the methodology used to calculate the failure probability of a population of TRISO particles. The methodology relies on a Monte Carlo approach where each particle corresponds to a set of statistically sampled parameters from the distributions of as-fabricated fuel characteristics (e.g., dimensions, densities, etc.) that can be found amongst the particles in a pebble. For each sample, BISON runs a 1D model of the particle over the required irradiation time. For particles with localized flaws (i.e., aspherical particles and particles with cracked IPyC), an adjustment of the maximum stress and mean strength will be made as described in Section 4.3.3.

At each time step, the following failure mechanisms are checked:

- Pressure vessel failure of a spherical or aspherical particle: Failure is assumed when the maximum tangential tensile stress induced in the $\mathrm{SiC}$ layer by internal gas pressure is greater than the mean strength of the $\mathrm{SiC}$.

- $\mathrm{SiC}$ failure due to IPyC cracking: Cracking of the IPyC is assumed when the maximum tangential tensile stress in the PyC layer is greater than its mean strength. A cracked IPyC changes stress state in the SiC layer. SiC failure is assumed when the maximum tangential tensile stress induced in the SiC layer by the cracked IPyC is greater than its mean strength.

In all cases, the maximum stress is compared to a strength that is sampled from a Weibull distribution (mean strength ms and modulus $\mathrm{m}$ ) to determine whether or not failure (i.e., IPyC cracking, SiC failure) occurs.

The methodology laid out in Figure 4.12 can also be used to simulate fission product diffusion for a collection of fuel particles. The fission product diffusion calculations combine the release fractions (release normalized to calculated 


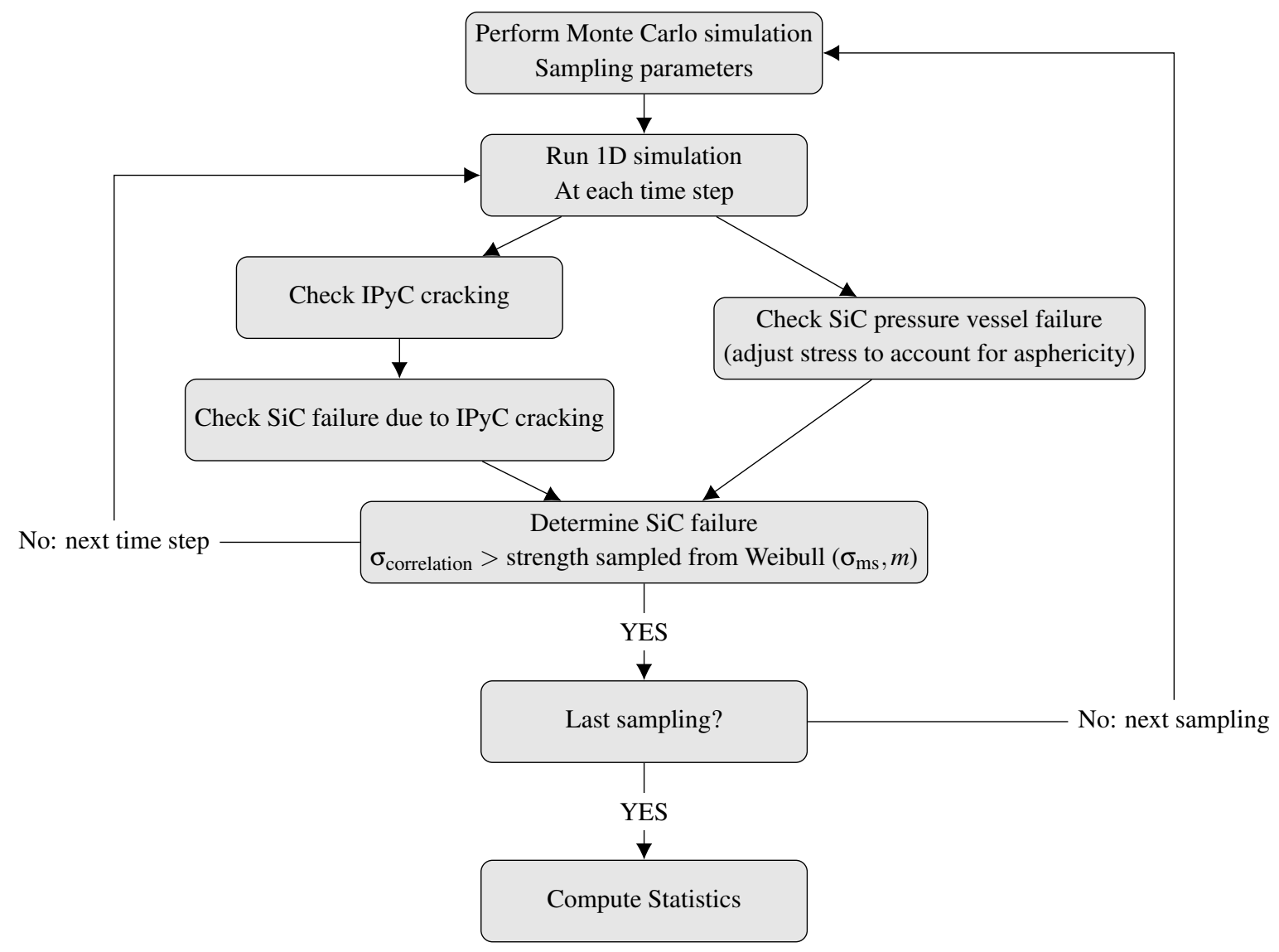

Figure 4.12: BISON Monte Carlo scheme for calculation of failure probability of TRISO particles.

inventory) of all TRISO particles in the Monte Carlo sample. Coating layers that have been determined failed by stress analysis are assigned a large diffusivity (e.g., $10^{-6} \mathrm{~m}^{2} / \mathrm{s}$ ) to model the loss of retention power of that layer. Release is calculated by Fickian diffusion, while inventory is obtained from fission rate density and fission yields.

\subsection{Demonstration problems}

\subsubsection{Incorporate diffusion coefficients from LANL}

The new diffusion coefficients are tested for a TRISO fuel particle under representative irradiation given in Table 4.9. The fuel parameters given in Table 4.10 are based on the AGR-5/6/7 fuel specification. The FGR (moles), internal pressure, and tangential stress of the $\mathrm{SiC}$ layer are plotted in Figure 4.13, Figure 4.14, and Figure 4.15, respectively. As seen from all three plots, at lowest temperature of $700^{\circ} \mathrm{C}$ and highest temperature of $1900^{\circ} \mathrm{C}$, the difference between new and old diffusion coefficients is invisible. For other temperatures, the FGR and pressure with new diffusion coefficients are smaller than the old ones. As a consequence, the compressive tangential stress in the SiC layer becomes higher at higher fluence as less internal pressure is built up by fission gas release. This means the pressure vessel failure is less likely to occur at the end of irradiation.

\subsubsection{Benchmark BISON against PARFUME}

In this example, BISON is compared to PARFUME code for a TRISO UCO fuel particle under representative irradiation conditions. These irradiation conditions are given in Table 4.11 . The temperature of $700^{\circ} \mathrm{C}, 1000^{\circ} \mathrm{C}$, and $1300^{\circ} \mathrm{C}$ are chosen to fall within the validity range of the material properties. The fuel parameters given in Table 4.10 are based on the AGR-5/6/7 fuel specification. A brief description of AGR-5/6/7 experiments is provided in the next section. 
Table 4.9: Irradiation conditions for Section 4.4.1.

\begin{tabular}{lcccc}
\hline Conditions & EFPD & $\begin{array}{c}\text { Burnup } \\
(\% \text { FIMA })\end{array}$ & $\begin{array}{c}\text { Fast fluence } \\
\left(\mathbf{x 1 0} \mathbf{n}^{25} \mathbf{m}^{2}\right), E>\mathbf{0 . 1 8} \mathbf{~ M e V}\end{array}$ & $\begin{array}{c}\text { Irradiation } \\
\text { temperature }\left({ }^{\circ} \mathbf{C}\right)\end{array}$ \\
\hline 1 & 500 & 13.5 & 5 & 700 \\
2 & 500 & 13.5 & 5 & 1000 \\
3 & 500 & 13.5 & 5 & 1300 \\
4 & 500 & 13.5 & 5 & 1600 \\
5 & 500 & 13.5 & 5 & 1900 \\
\hline
\end{tabular}

Table 4.10: Fuel parameters.

\begin{tabular}{llc}
\hline Category & \multicolumn{1}{c}{ Parameter } & Nominal values \\
\hline \multirow{4}{*}{ Fuel characteristics } & Carbon/uranium (atomic ratio) & 15.5 \\
& Oxygen/uranium (atomic ratio) & 0.4 \\
& Kernel diameter $(\mu \mathrm{m})$ & 1.5 \\
\hline \multirow{5}{*}{ Particle geometry } & 425 \\
& Buffer thickness $(\mu \mathrm{m})$ & 100 \\
& IPyC/OPyC thickness $(\mu \mathrm{m})$ & 40 \\
& SiC thickness $(\mu \mathrm{m})$ & 35 \\
\hline \multirow{5}{*}{ Fuel properties } & Kernel density $\left(\mathrm{g} / \mathrm{cm}^{3}\right)$ & 11.0 \\
& Kernel theoretical density $\left(\mathrm{g} / \mathrm{cm}^{3}\right)$ & 11.4 \\
& Buffer density $\left(\mathrm{g} / \mathrm{cm}^{3}\right)$ & 1.05 \\
& Buffer theoretical density $\left(\mathrm{g} / \mathrm{cm}^{3}\right)$ & 2.25 \\
& IPyC density $\left(\mathrm{g} / \mathrm{cm}^{3}\right)$ & 1.90 \\
& OPyC density $\left(\mathrm{g} / \mathrm{cm}^{3}\right)$ & 1.90 \\
& IPyC/OPyC BAF & 1.05 \\
\hline
\end{tabular}

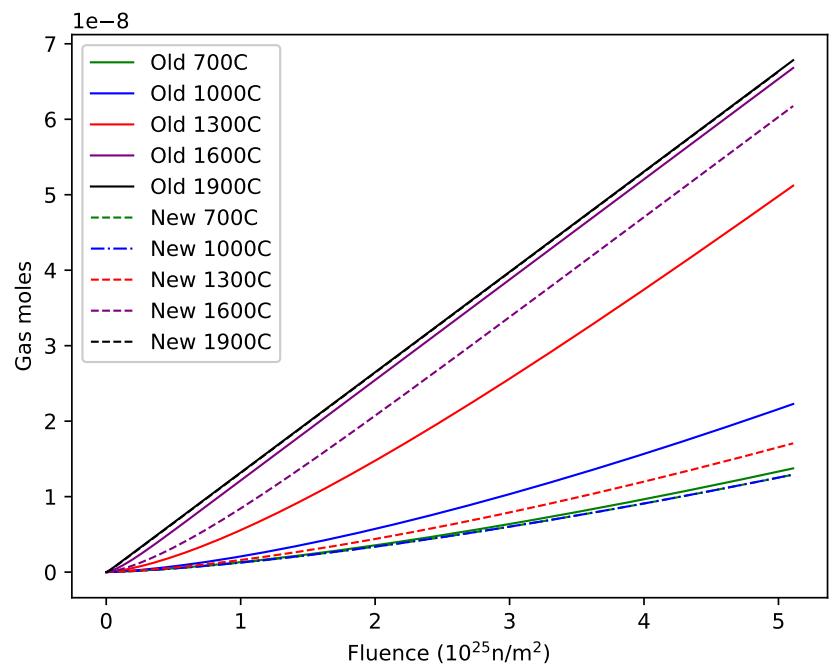

Figure 4.13: Fission gas realease (moles).

For each of the three conditions, the tangential stress at inner and outer of each layers are plotted in Figure 4.16. The tangential stress are tensile in the IPyC and OPyC layer while compressive in the SiC layer. The extremum tensile stress in both PyC layers and compressive stress in the $\mathrm{SiC}$ layer decreases with the increase of temperature due to 


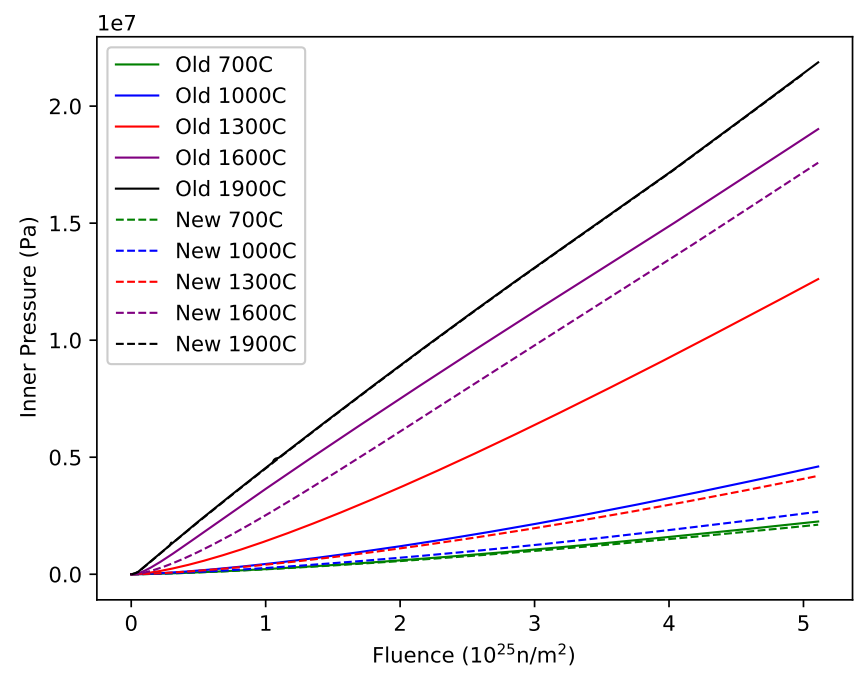

Figure 4.14: Inner pressure $(\mathrm{Pa})$.

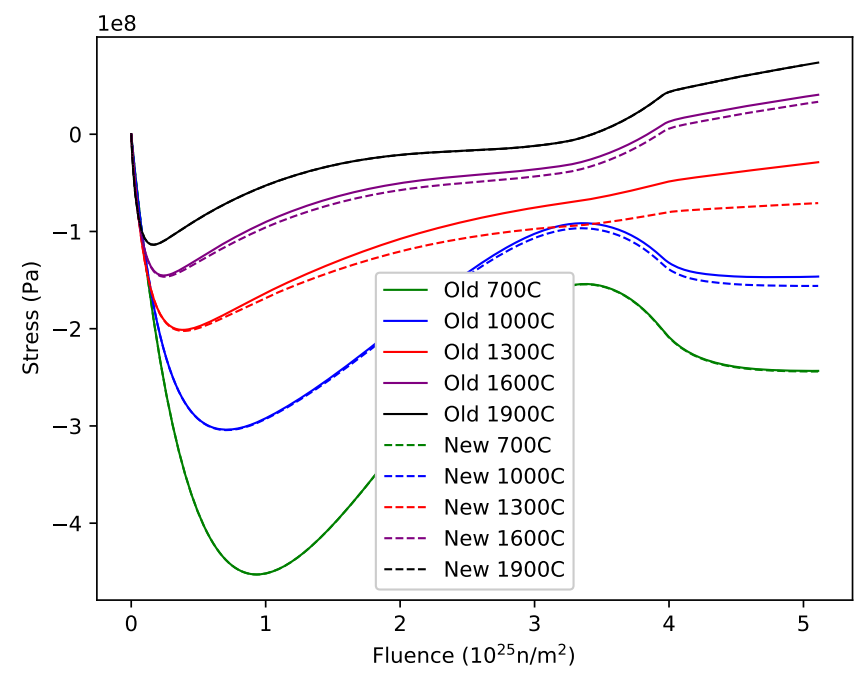

Figure 4.15: Tangential stress at the inner surface of the $\mathrm{SiC}$ layer.

irradiation creep. The internal pressure that results in increasing tensile stress in the $\mathrm{SiC}$ layer is built up by fission gases in the buffer-IPyC gap. The fission gases moles in the gap is plotted in Figure 4.16d. All of those results show good agreement between BISON and PARFUME.

Table 4.11: Irradiation conditions for Section 4.4.2.

\begin{tabular}{lcccc}
\hline Conditions & EFPD & $\begin{array}{c}\text { Burnup } \\
(\% \text { FIMA })\end{array}$ & $\begin{array}{c}\text { Fast fluence } \\
\left(\mathbf{x 1 0} \mathbf{n}^{25} \mathbf{m}^{2}\right), E>\mathbf{0 . 1 8} \mathbf{~ M e V}\end{array}$ & $\begin{array}{c}\text { Irradiation } \\
\text { temperature }\left({ }^{\circ} \mathbf{C}\right)\end{array}$ \\
\hline 1 & 500 & 13.5 & 5 & 700 \\
2 & 500 & 13.5 & 5 & 1000 \\
3 & 500 & 13.5 & 5 & 1300 \\
\hline
\end{tabular}




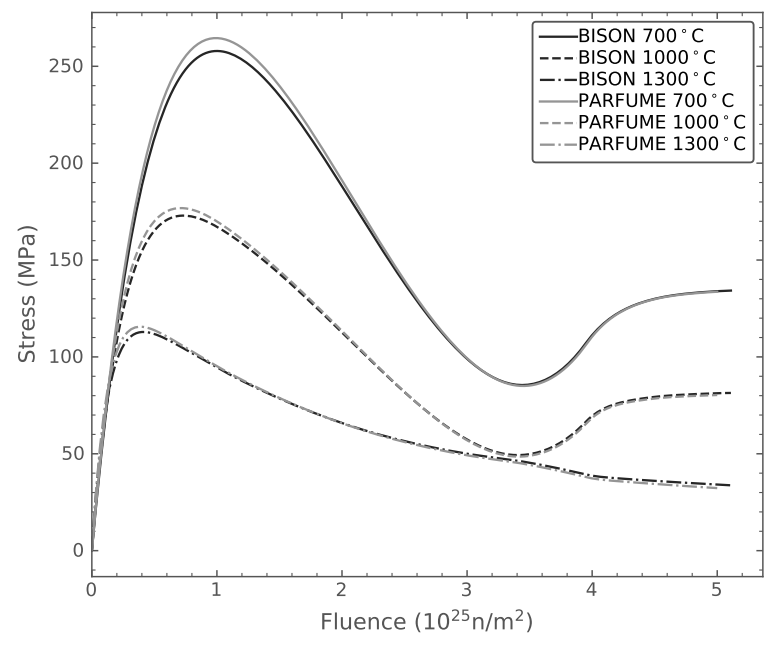

(a) Inner wall tangential stress for IPyC.

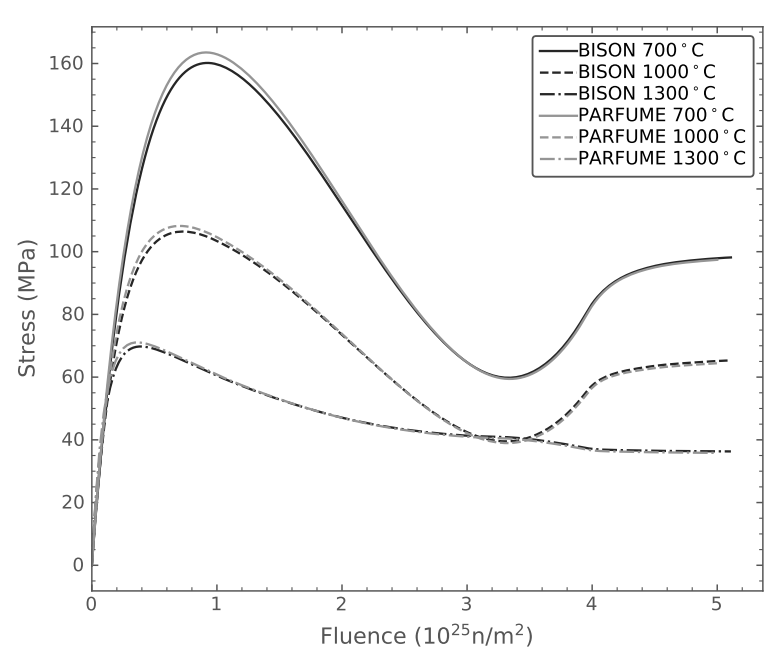

(c) Inner wall tangential stress for OPyC.

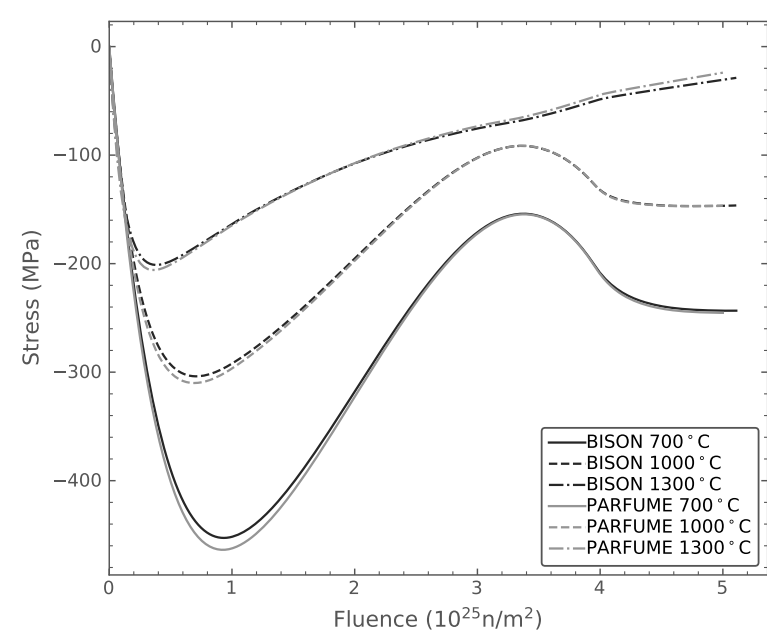

(b) Inner wall tangential stress for $\mathrm{SiC}$.

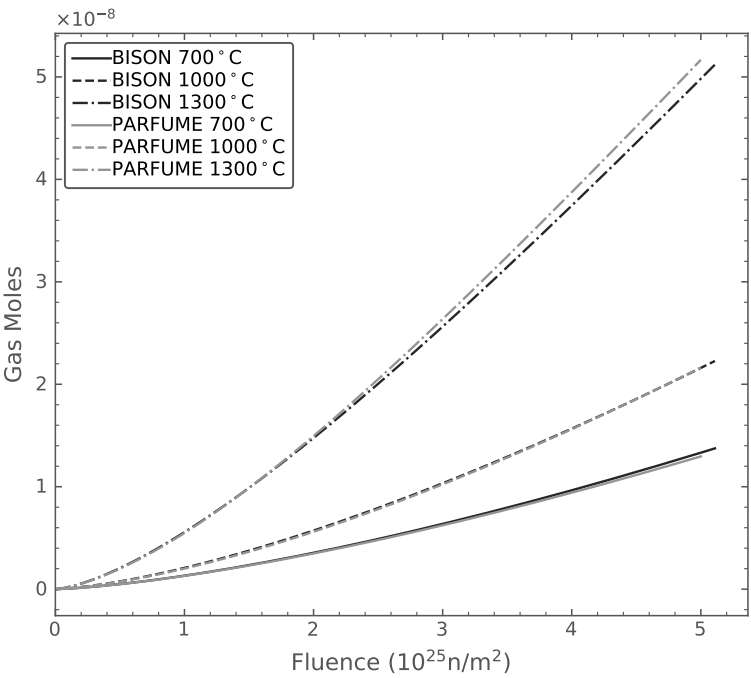

(d) Fission gases moles in the buffer-IPyC gap.

Figure 4.16: BISON-PARFUME comparison for benchmark problems. 
The failure probability of a TRISO UCO fuel particle under the irradiation conditions given Table 4.11 is calculated using a Monte Carlo scheme. The statistical variation of particle dimensions and material properties is listed in Table 4.12. The stress correlation functions and effective mean strength are precomputed for each condition, given in Table 4.13. BISON and PARFUME are used to compute probability of cracking fo the IPyC layer and SiC failure. Both codes use the same stress correlation factors and effective mean strength listed in Table 4.13. Results are reported in Table 4.14 and they show decent agreement. It is worthwhile to mention that the SiC failure probability of condition 3 is too low such that the required number of Monte Carlo samples is beyond BISON's current computation limit as 100 million samples. A more efficient approach to calculate low failure probability will be developed in the future.

Table 4.12: Statistical variation of fuel parameters for benchmark problems.

\begin{tabular}{llc}
\hline Category & \multicolumn{1}{c}{ Parameter } & $\begin{array}{c}\text { Nominal values } \\
\text { Standard Deviation }\end{array}$ \\
\hline \multirow{4}{*}{ Particle geometry } & Kernel diameter $(\mu \mathrm{m})$ & $425 \pm 10$ \\
& Buffer thickness $(\mu \mathrm{m})$ & $100 \pm 10$ \\
& IPyC/OPyC thickness $(\mu \mathrm{m})$ & $40 \pm 3$ \\
& SiC thickness $(\mu \mathrm{m})$ & $35 \pm 2$ \\
& Particle asphericity $(\mathrm{SiC}$ aspect ratio) & 1.04 \\
\hline \multirow{3}{*}{ Fuel properties } & IPyC density $\left(\mathrm{g} / \mathrm{cm}^{3}\right)$ & $1.90 \pm 0.02$ \\
& OPyC density $\left(\mathrm{g} / \mathrm{cm}^{3}\right)$ & $1.90 \pm 0.02$ \\
& IPyC/OPyC BAF & $1.05 \pm 0.005$ \\
\hline
\end{tabular}

Table 4.13: Stress correlation factors and effective mean strength for benchmark problems.

\begin{tabular}{|c|c|c|c|c|c|c|}
\hline \multirow{2}{*}{ Conditions } & \multirow{2}{*}{$\begin{array}{c}\text { Intact } \\
\sigma_{\mathrm{ms}}(\mathrm{MPa})\end{array}$} & \multicolumn{2}{|c|}{ IPyC cracking } & \multicolumn{3}{|c|}{ Asphericity } \\
\hline & & $\sigma_{\mathrm{ms}}(\mathrm{MPa})$ & $\frac{\bar{\sigma}_{2 \mathrm{D}}}{\overline{\bar{\sigma}}_{1 \mathrm{D}}}$ & $\sigma_{\mathrm{ms}}(\mathrm{MPa})$ & $\frac{\bar{\sigma}_{2 \mathrm{D}}}{\bar{\sigma}_{1 \mathrm{D}}}$ & $\frac{\Delta \bar{\sigma}_{2 \mathrm{D}}}{\Delta \bar{\sigma}_{1 \mathrm{D}}}$ \\
\hline 1 & 711 & 1198 & -0.87 & 993 & 1.41 & 1.22 \\
\hline 2 & 719 & 1198 & -0.97 & 1041 & 1.59 & 1.41 \\
\hline 3 & 719 & 1198 & -0.97 & 1095 & 1.60 & 1.48 \\
\hline
\end{tabular}

Table 4.14: Failure probability for benchmark problems.

\begin{tabular}{ccccc}
\hline \multirow{2}{*}{ Conditions } & \multicolumn{2}{c}{ BISON } & \multicolumn{2}{c}{ PARFUME } \\
\cline { 2 - 5 } & IPyC cracking & SiC failure & IPyC cracking & SiC failure \\
\hline 1 & $9.11 \times 10^{-1}$ & $0.75 \times 10^{-3}$ & $8.94 \times 10^{-1}$ & $1.03 \times 10^{-3}$ \\
2 & $7.10 \times 10^{-2}$ & $6.10 \times 10^{-6}$ & $5.48 \times 10^{-2}$ & $7.80 \times 10^{-6}$ \\
3 & $0.80 \times 10^{-3}$ & $1.00 \times 10^{-8}$ & $1.16 \times 10^{-3}$ & $1.64 \times 10^{-8}$ \\
\hline
\end{tabular}

\subsubsection{AGR-5/6/7 Irradiation Test Predictions}

DOE's Advanced Gas Reactor program planned a series of irradiation experiments on TRISO fuel. The AGR-5/6/7 experiments test TRISO particles with low-enriched UCO fuel. The AGR-5/6/7 test train houses 5 capsules, with each capsule containing compacts filled with TRISO particles. The TRISO particle geometry and material properties are the same as listed in Table 4.10 and Table 4.12. The details of these experiments can be found in [15].

Failure probability calculations were performed on three compacts in Capsule 5. Their irradiation conditions are listed in Table 4.15. For these compacts, the inner-wall tangential stresses in $\mathrm{SiC}$ of an intact particle, particle with IPyC cracking and aspherical particle are plotted in Figure 4.17. The evolution of the buffer outer and IPyC inner 
Table 4.15: Irradiation conditions for AGR-5/6/7 Compact 5-1-3, 5-2-3 and 5-6-1.

\begin{tabular}{lcccc}
\hline Compact & EFPD & $\begin{array}{c}\text { Burnup } \\
(\% \text { FIMA })\end{array}$ & $\begin{array}{c}\text { Fast fluence } \\
\left(\times 10^{25} \mathbf{n} / \mathbf{m}^{2}\right)\end{array}$ & $\begin{array}{c}\text { Irradiation } \\
\text { temperature }\left({ }^{\circ} \mathbf{C}\right)\end{array}$ \\
\hline $5-1-3$ & 510 & 12.67 & 4.54 & 803 \\
$5-2-3$ & 510 & 12.17 & 4.19 & 812 \\
$5-6-1$ & 510 & 8.24 & 2.25 & 696 \\
\hline
\end{tabular}

radii and buffer-IPyC gap is plotted in Figure 4.18. Since the buffer-IPyC gap contributes a significant fraction of the thermal resistance in a TRISO particle, increasing gap size ought to result in increased temperature in the fuel kernel.

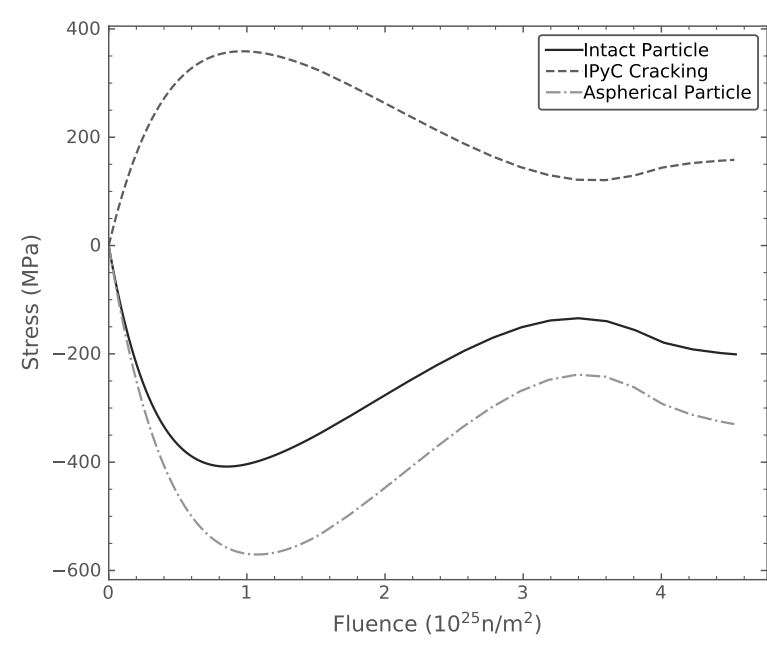

(a) Compact 5-1-3.

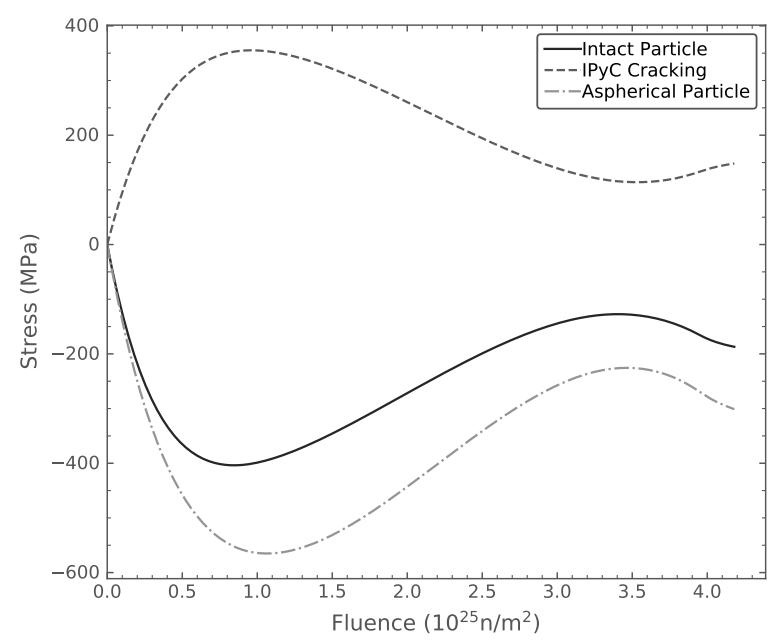

(b) Compact 5-2-3.

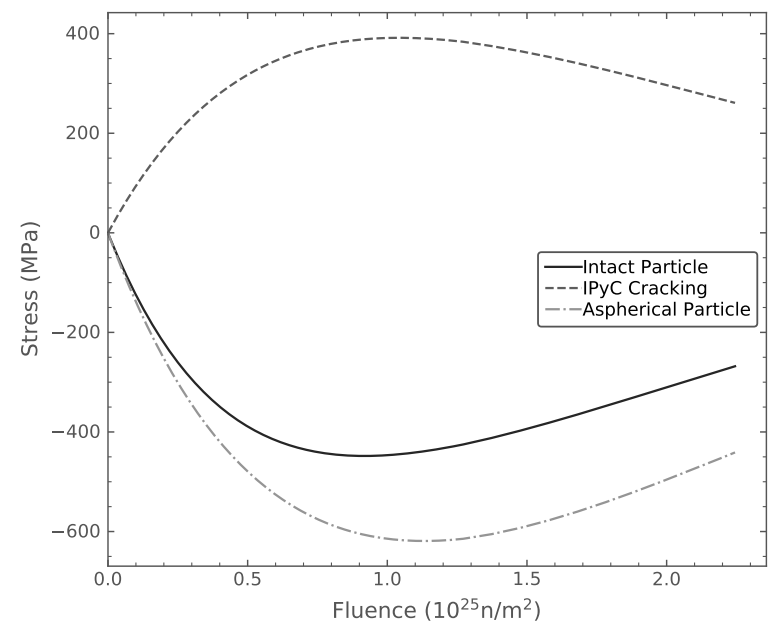

(c) Compact 5-6-1.

Figure 4.17: Inner-wall tangential stress in $\mathrm{SiC}$ for intact particle, particle with IPyC cracking and aspherical particle in AGR-5/6/7 Compact 5-1-3, 5-2-3 and 5-6-1.

The stress correlation factors and effective mean strength were precomputed for each compact by BISON axisymmetric models, and their values are provided in Table 4.16. Table 4.17 summarizes the failure probabilities of IPyC cracking and $\mathrm{SiC}$ failure. The evolution of $\mathrm{SiC}$ failure probability is shown in Figure 4.19. The $\mathrm{SiC}$ failures of all three compacts in Capsule 5 are due to IPyC cracking, causing localized tensile stress in the SiC layer. Compact 5-6-1 has 


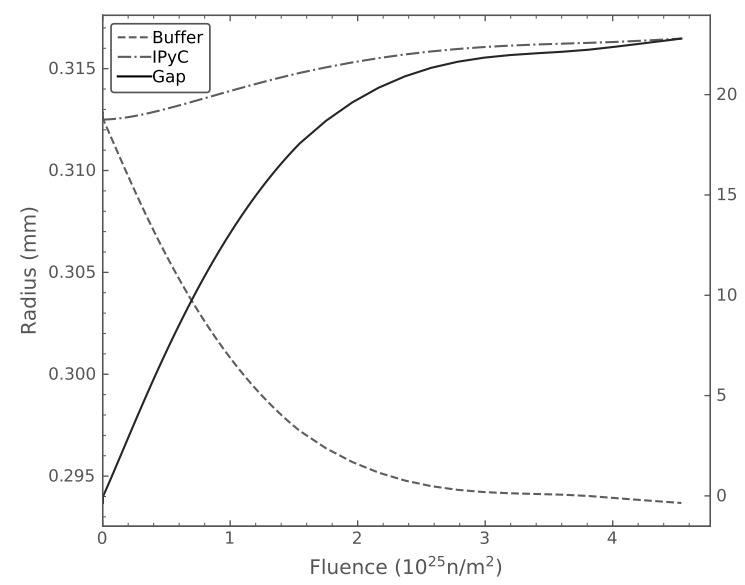

(a) Compact 5-1-3.

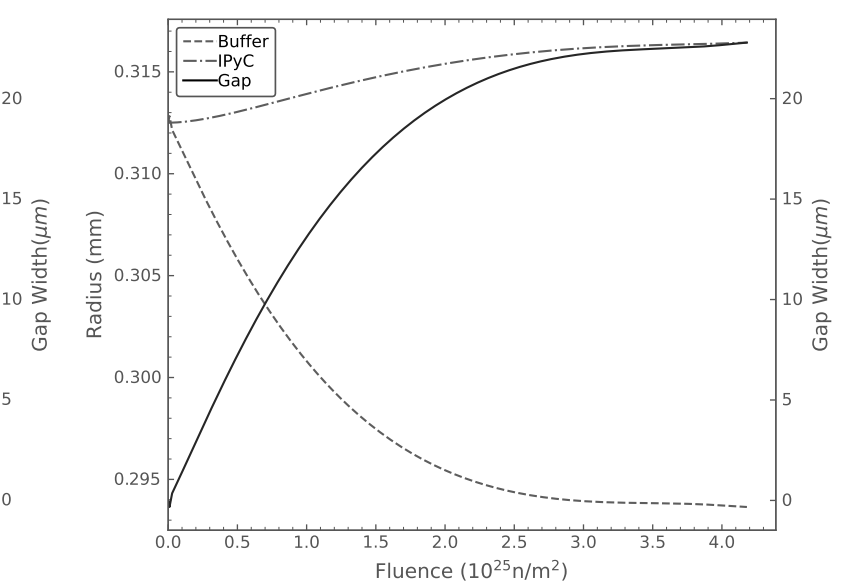

(b) Compact 5-2-3.

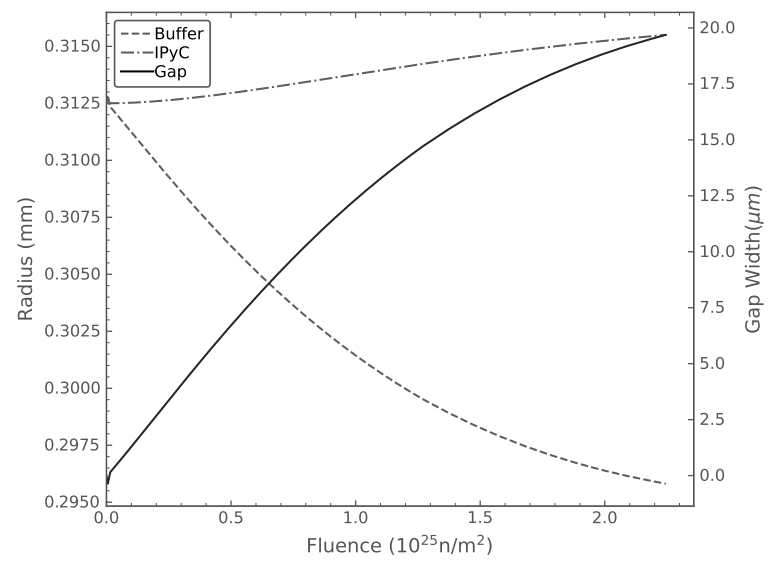

(c) Compact 5-6-1.

Figure 4.18: Buffer outer and IPyC inner radii for AGR-5/6/7 Compact 5-1-3, 5-2-3 and 5-6-1. Gap is computed as their difference. 
the highest $\mathrm{SiC}$ failure probability because of its low irradiation temperature that results in a high tensile stress in $\mathrm{SiC}$ layer in the presence of IPyC cracking. Assuming 2,275 fuel particles per compact, the estimated number of failed particle is 1, 2, and 1 in Compacts 5-1-3, 5-2-3, and 5-6-1, respectively. This is the same number of failed particles per compact that PARFUME predicted [15].

Table 4.16: Stress correlation factors and effective mean strength for AGR-5/6/7 Compact 5-1-3, 5-2-3 and 5-6-1.

\begin{tabular}{ccccccc}
\hline \multirow{2}{*}{ Compact } & Intact & \multicolumn{2}{c}{ IPyC cracking } & \multicolumn{3}{c}{ Asphericity } \\
\cline { 2 - 7 } & $\sigma_{\mathrm{ms}}(\mathrm{MPa})$ & $\sigma_{\mathrm{ms}}(\mathrm{MPa})$ & $\overline{\bar{\sigma}}_{2 \mathrm{D}}$ & $\bar{\sigma}_{\mathrm{ms}}(\mathrm{MPa})$ & $\frac{\bar{\sigma}_{2 \mathrm{D}}}{\bar{\sigma}_{1 \mathrm{D}}}$ & $\frac{\Delta \bar{\sigma}_{2 \mathrm{D}}}{\Delta \bar{\sigma}_{1 \mathrm{D}}}$ \\
\hline $5-1-3$ & 710 & 1199 & -0.96 & 988 & 1.53 & 1.33 \\
$5-2-3$ & 711 & 1199 & -0.96 & 998 & 1.53 & 1.34 \\
$5-6-1$ & 708 & 1199 & -0.96 & 1034 & 1.51 & 1.06 \\
\hline
\end{tabular}

Table 4.17: Failure probability for AGR-5/6/7 Compact 5-1-3, 5-2-3 and 5-6-1.

\begin{tabular}{ccc}
\hline \multirow{2}{*}{ Compact } & \multicolumn{2}{c}{ BISON } \\
\cline { 2 - 3 } & IPyC cracking & SiC failure \\
\hline $5-1-3$ & $7.45 \times 10^{-1}$ & $7.34 \times 10^{-4}$ \\
$5-2-3$ & $6.55 \times 10^{-1}$ & $4.90 \times 10^{-4}$ \\
$5-6-1$ & $9.15 \times 10^{-1}$ & $1.31 \times 10^{-3}$ \\
\hline
\end{tabular}

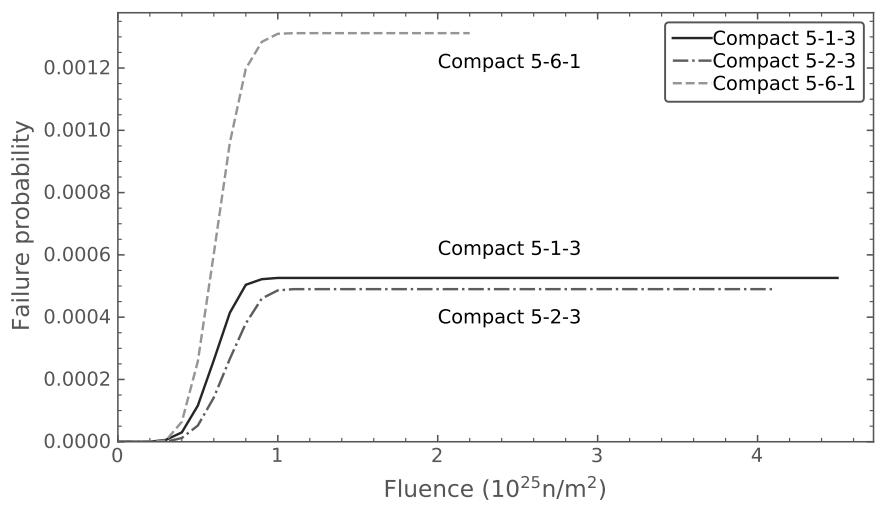

Figure 4.19: Evolution of SiC failure probability for AGR-5/6/7 Compact 5-1-3, 5-2-3 and 5-6-1.

The normalized histograms of statistically varying parameters for failed particles in Compact 5-1-3 are shown in Figure 4.20. A fitted probability density function of each parameter for failed particles is compared to the probability density function for all particles. As can be seen from those plots, there is a significant change between the probability density function of failed particles and all particles in IPyC thickness and SiC thickness. The changes for other parameters are relatively small, which indicates that those parameters have little effect on particle failures. The mean value of $\mathrm{SiC}$ thickness for failed particle is smaller compared to that for all particles. This implies that the thinner SiC layer results in more particles failure. This is due to the decrease in SiC thickness causing a higher tangential stress in the layer. Conversely, the mean value of IPyC thickness for failed particle is larger compared to that for all particles. This means that as the IPyC thickness increases, particle failure is more likely to happen. It is because as IPyC becomes thicker, the irradiation-induced shrinkage increases and subsequent increases IPyC cracking probability as well as $\mathrm{SiC}$ failure probability due to IPyC cracking. It is interesting to see similar findings obtained from a sensitivity study on failure probability[16]. 


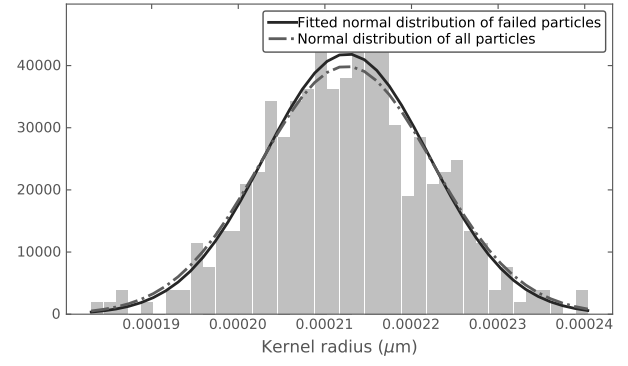

(a) Kernel radius.

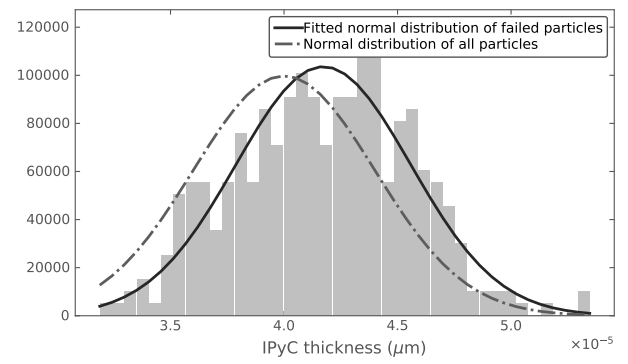

(c) IPyC thickness.

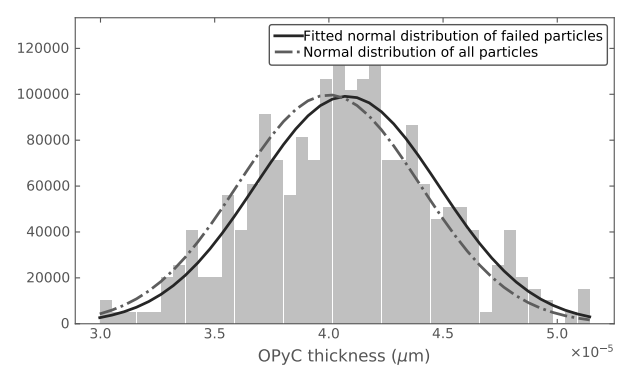

(e) OPyC thickness.

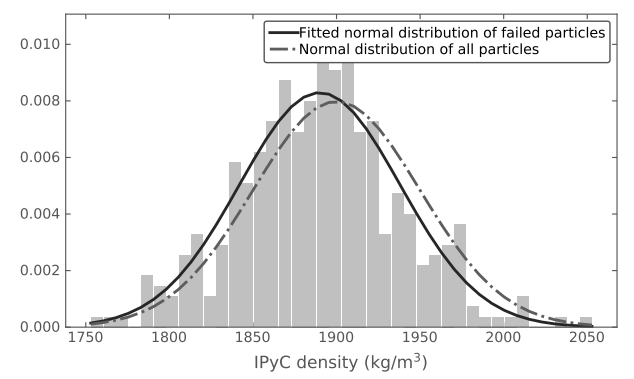

(g) IPyC density.

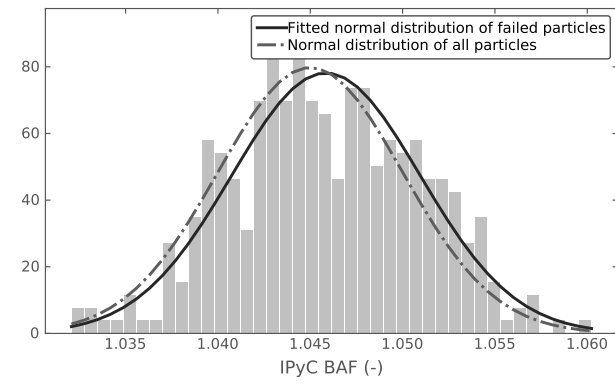

(i) IPyC BAF.

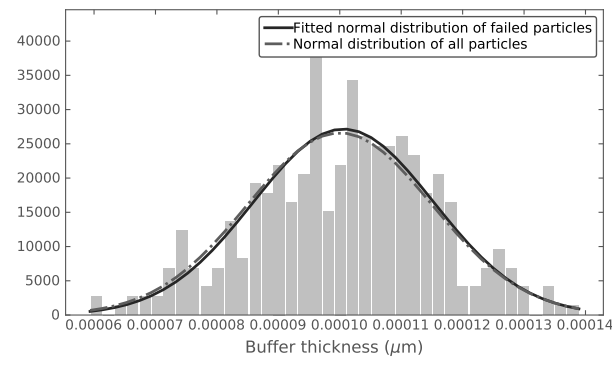

(b) Buffer thickness.

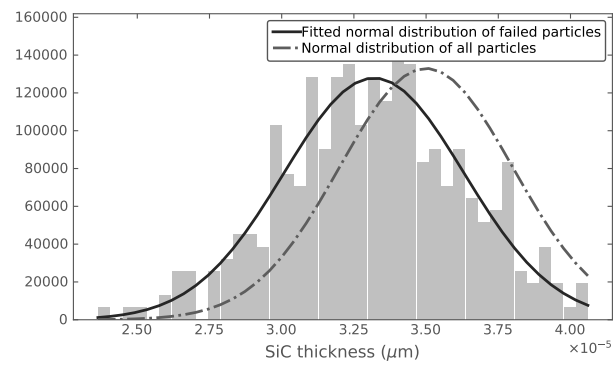

(d) $\mathrm{SiC}$ thickness.

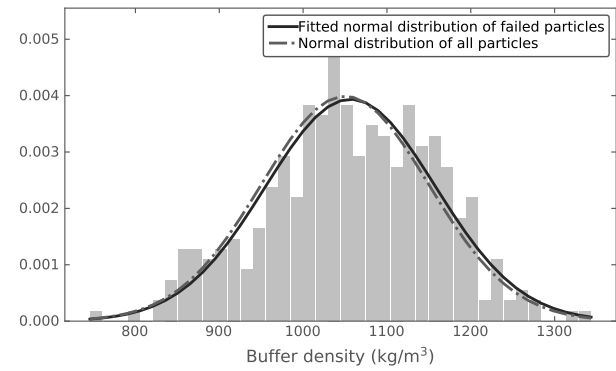

(f) Buffer density.

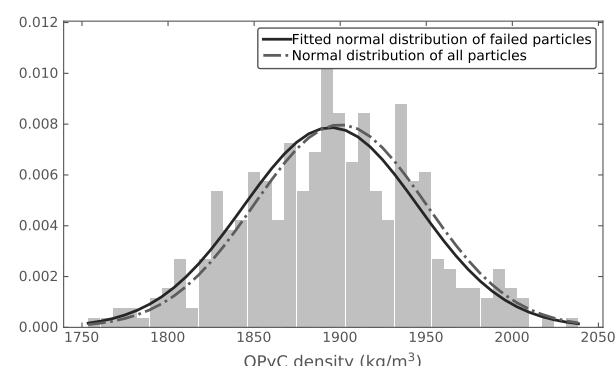

(h) OPyC density.

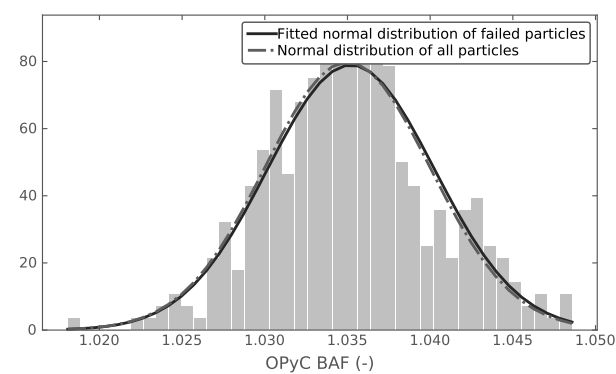

(j) OPyC BAF.

Figure 4.20: Normalized histograms of statistically varying parameters for failed particles in AGR-5/6/7 Compact 5-1-3. 


\subsection{Conclusions}

New TRISO material models have been implemented in BISON to expand its ability to model fuel performance of TRISO particles. These models include mechanical (elastic, creep, irradiation strain), thermal (thermal conductivity, specific heat), mass diffusion, and fission gas production and release models. For fission gas release model, new diffusion coefficients from the LANL's Centipede simulations were tested for on a TRISO fuel particle under representative irradiation conditions in BISON. At moderate temperatures between $700^{\circ} \mathrm{C}$ and $1900^{\circ} \mathrm{C}$, the compressive tangential stress in the $\mathrm{SiC}$ layer becomes higher at higher fluence as less internal pressure is built up by fission gas release. This causes pressure vessel failure to be less likely to occur. Another important addition is the ability to perform statistical failure analysis of large samples of fuel particles. This new capability, which continues to grow, enables evaluation of failure due to pressure or crack formation by analyzing many thousands of particles. This enables realistic calculations of fission product release from the many particles in a TRISO-fueled reactor. Benchmark problems were developed to compare BISON and PARFUME. Results show a decent agreement in stress level and failure probability. BISON is used to simulate three compacts in AGR-5/6/7 and compute their failure probabilities. The statistics of failed particles indicates that as IPyC and SiC thickness have a significant effect on the particle failures.

\subsection{Future Work}

Future work will involve simulating other failure mechanisms and incorporating them in the Monte Carlo scheme. Fast failure probability calculation approach will be developed to handle low-failure probability case for which Monte Carlo needs very large sample size. Additional developments will add graphite matrix diffusion to fission product release calculation and include them into statistical failure analysis. 


\section{Bibliography}

[1] Darrell L. Knudson, Gregory K Miller, G.K. Miller, D.A. Petti, J.T. Maki, and D.L. Knudson. Parfume theory and model basis report. 92009.

[2] H. Nabielek, K. Fukuda, K. Minato, and T. Ogawa. Calculation of Particle Temperatures in NSRR Tests. Unpublished draft, Japanese Atomic Energy Agency, March 1992.

[3] J.K. Fink. Thermophysical properties of uranium dioxide. Journal of Nuclear Materials, 279:1-18, 2000.

[4] D. R. Olander. Fundamental aspects of nuclear reactor fuel elements. Technical Information Center, Energy Research and Development Administration, 1976.

[5] G.K. Miller, D.A. Petti, J.T. Maki, D.L. Knudson, , and W.F. Skerjanc. PARFUME Theory and Model Basis Report. Report INL/EXT-08-14497 (Rev.1), Idaho National Laboratory, September 2018.

[6] F. Ho. NP-MHTGR: Material Models of Pyrocarbon and Pyrolytic Silicon Carbide. Report CEGA-002820 Rev. 1, CEGA Corporation, July 1993.

[7] V. Barabash, I. Mazul, R. Latypov, A. Pokrovsky, and C.H. Wu. The effect of low temperature neutron irradiation and annealing on the thermal conductivity of advanced carbon-based materials. Journal of Nuclear Materials, 307-311:1300-1304, 2002.

[8] D. Petti, P. Martin, M. Phelip, and R. Ballinger. Development of improved models and designs for coatedparticle gas reactor fuels. Technical Report INL/EXT-05-02615, Idaho National Engineering and Environmental Laboratory, December 2004.

[9] J. J. Powers and B. D. Wirth. A review of TRISO fuel performance models. J Nuclear Materials, 405(1):74-82, 2010.

[10] J.D. Hales, R.L. Williamson, S.R. Novascone, D.M. Perez, B.W. Spencer, and G. Pastore. Multidimensional multiphysics simulation of triso particle fuel. Journal of Nuclear Materials, 443(1):531 - 543, 2013.

[11] L. L. Snead, T. Nozawa, Y. Katoh, T.-S. Byun, S. Kondo, and D. A. Petti. Handbook of sic properties for fuel performance modeling. Journal of Nuclear Materials, 371:329-377, 2007.

[12] U. Littmark and J.F. Ziegler. Handbook of Range Distributions for Energetic Ions in All Elements. Technical report, Pergamon Press, 1980.

[13] A.H. Booth. A method of calculating gas diffusion from $\mathrm{UO}_{2}$ fuel and its application to the X-2-f loop test. Technical Report AECL-496, Atomic Energy of Canada Ltd., 1957.

[14] J.A. Turnbull, C.A. Friskney, J.R. Findlay, F.A. Johnson, and A.J. Walter. The diffusion coefficients of gaseous and volatile species during the irradiation of uranium dioxide. Journal of Nuclear Materials, 107:168-184, 1982.

[15] W.F. Skerjanc. AGR-5/6/7 Irradiation Test Predictions using PARFUME. Report INL/EXT-17-43189 (Rev.0), Idaho National Laboratory, September 2017.

[16] William F. Skerjanc, John T. Maki, Blaise P. Collin, and David A. Petti. Evaluation of design parameters for triso-coated fuel particles to establish manufacturing critical limits using parfume. Journal of Nuclear Materials, 469:99 - 105, 2016. 


\section{Chapter 5}

\section{DEVELOP AND DEMONSTRATE INITIAL CAPABILITY TO SIMULATE UN/UC FUEL}

D. S. Stafford

C. Matthews

This section documents the developments in BISON related to fuel performance modeling of Uranium Nitride (UN) and Uranium Carbide (UC) fuel types. UN/UC fuels have been proposed for use in advanced of various types (e.g., high temperature gas reactors and liquid metal fast breeder reactors). Both materials can be pressed and sintered from a powder form into appropriate shapes for specific reactor types. UN/UC materials typically have higher thermal conductivity, metal density, and melting points compared to oxide fuels (i.e., $\mathrm{UO}_{2}$ ). These increased material properties allow for a higher power density and better thermal performance compared to $\mathrm{UO}_{2}$.

However, UN/UC fuels have not been investigated and used as widely as oxide fuels. A number of behaviors that can be strongly influenced by irradiation and temperature (swelling, creep, fission product production and migration, etc...) are not well understood. The available material models from earlier research are being incorporated into BISON and will be used as a starting point for developing fuel performance models for UN/UC applications. BISON will be updated with improved models as more recent experimental investigations of UN/UC fuels become available.

\subsection{Overview}

We added material models for thermo-mechanical properties of ( $\mathrm{U}, \mathrm{Pu}) \mathrm{C}$ (mixed carbides) and $(\mathrm{U}, \mathrm{Pu}) \mathrm{N}$ (mixed nitrides) to BISON. The new carbide models are:

- ADMCElasticityTensor

- ADMCCreepUpdate

- ADMCThermal

- ADMCThermalExpansionEigenstrain

- ADMCVolumetricSwellingEigenstrain

The new nitride models are:

- ADMNElasticityTensor

- ADMNCreepUpdate

- ADMNThermal

- ADMNThermalExpansionEigenstrain 


\section{- ADMNVolumetricSwellingEigenstrain}

The new models use automatic differentiation (AD) so that they can be used efficiently with the single matrix preconditioner (SMP) mode of MOOSE, thus enabling efficient convergence of the nonlinear solve within a few steps. These material models are fully documented in the BISON code documentation and are verified against hand calculations to ensure that the results match published results.

Models were derived from various sources, but lean heavily on Preusser's selection of correlations for carbides [1], and on a series of experimental results by Hayes for nitries [2] [3] [4] [5].

Two test cases were designed that demonstrate combined utilization of the carbide and the nitride material models. Both test cases are based on experiments run at EBR-II in the 1970s and 1980s. These test cases are currently set up as assessment cases, although there is insufficient data to fully validate the results.

\subsection{Mixed Carbides}

\subsubsection{Thermal Properties}

The material model ADMCThermal calculates the thermal conductivity $\lambda$ and specific heat capacity $c_{p}$ of $\mathrm{U}_{1-x} \mathrm{Pu}_{x} \mathrm{C}$, where $x$ refers to the atomic ratio of $\mathrm{Pu}$ to $\mathrm{U}$. Input is given as $X_{P u C}$, which is the mole fraction of $\mathrm{PuC}$ and is therefore equivalent to $x$. When weight fractions are required for individual models, ADMCThermal internally converts the atom fractions into weight fractions.

The thermal conductivity $\lambda$ is given in $\mathrm{W} / \mathrm{m}-\mathrm{K}$ by [6], Eqs. $4-28 \mathrm{a}$ and $\mathrm{b}$, which are independent of $\mathrm{X}_{P u C}$ in the range $323 \mathrm{~K}$ to $2573 \mathrm{~K}$ :

$$
\begin{gathered}
\lambda=17.5-5.65 \times 10^{-3}(T-273)+8.14 \times 10^{-6}(T-273)^{2} \\
323<T<773 \mathrm{~K} \\
\lambda=12.76+8.71 \times 10^{-3}(T-273)-1.88 \times 10^{-6}(T-273)^{2} \\
773<T<2573 \mathrm{~K}
\end{gathered}
$$

A porosity correction is applied according to [6]:

$$
\begin{gathered}
\lambda(T, x, \phi)=\lambda_{0}(T, x) f(\phi) \\
f(\phi)=\frac{1-\phi}{1+\phi}
\end{gathered}
$$

Here, $\lambda$ is the thermal conductivity of the fuel as a function of temperature $T$, composition $x$, and porosity $\phi$, given the fresh-fuel thermal conductivity $\lambda_{0}$, and the porosity correction $f$.

Specific heat capacity $c_{p}$ is calculated in $\mathrm{J}$-mol/K as a function of temperature and of $x$ according to the specific heats of UC and $\mathrm{PuC}_{0.84}$ suggested by [6], Table 4-19:

$$
\begin{aligned}
& c_{p, \mathrm{UC}}=50.984+2.572 \times 10^{-2} T-1.868 \times 10^{-5} T^{2}+5.716 \times 10^{-9}-\frac{6.187 \times 10^{5}}{T} \\
& c_{p, \mathrm{PuC}_{0.84}}=57.876-1.4497 \times 10^{-2} T+7.7085 \times 10^{-6} T^{2}+8.6156 \times 10^{-9}-\frac{6.5548 \times 10^{5}}{T}
\end{aligned}
$$

However, although Preusser summarizes available UC and (U,Pu)C data with a linear fit [1], Blank questions the accuracy of the available data for mixed carbide and suggests that $(\mathrm{U}, \mathrm{Pu}) \mathrm{C}$ specific heat must lie between those of pure $\mathrm{UC}$ and $\mathrm{PuC}$ [6]. Therefore, in the absence of undisputed data, we mix the specific heats $c_{p, \mathrm{UC}}$ and $c_{p, \mathrm{PuC}_{0.84}}$ by

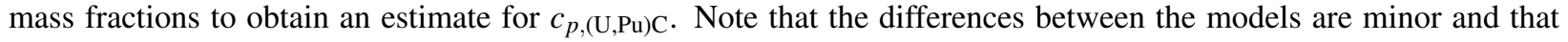
precise values of $c_{p}$ are not critical for modeling fuel rods in normal service. 


\subsubsection{Elasticity}

The ADMCElasticityTensor material model computes the elasticity tensor for mixed (U,Pu)C (monocarbides) using temperature- and porosity-dependent values for the Young's modulus and Poission's ratio as reported by [1]. The Young's modulus, $E$, is computed in units of $\mathrm{Pa}$ as:

$$
E=2.15 \times 10^{11}(1-a \phi)\left(1-0.92 \times 10^{-4}(T-273)\right)
$$

where $T$ is the temperature in $\mathrm{K}, \phi$ is the porosity, and $a$ is a correction coefficient for porosity that depends on the pore shape and composition of the fuel. A value of $a=2.3$ is suggested for $\mathrm{UC}$ and $a=1.54$ for $\left(\mathrm{U}_{0.8} \mathrm{Pu}_{0.2}\right) \mathrm{C}$ [1]. However, no data exists on the shape of the pores, and Blank claims that Pu content does not appreciably change $E$ [6]. Therefore, we take $a=2.3$.

For consistent property calculations, the correlation for Poisson's ratio $v$ must be computed from the same data as $E$, so we again use [1]:

$$
v=0.288-0.286 \phi
$$

This material is assumed to be isotropic, and $E$ and $v$ are therefore used to generate an isotropic elasticity tensor. The values for $E$ and $v$ are given over $0.05 \leq \phi \leq 0.27$, so a code exception (i.e., runtime error) is thrown if the porosity is outside of this range.

\subsubsection{Thermal Expansion}

The ADMCThermalExpansionEigenstrain material model computes an eigenstrain tensor that accounts for thermal expansion in mixed (U,Pu)C (monocarbides). This model uses the correlation of [1] to compute the coefficient of linear thermal expansion $\lambda$

$$
\lambda=1.007 \times 10^{-5}+1.17 \times 10^{-9} \mathrm{~T}
$$

where $T$ is temperature in $\mathrm{K}$ and $\lambda$ is given in $1 / \mathrm{K}$. The equation is independent of porosity because porosity does not have a significant influence on thermal expansion [6]. Likewise, the U/Pu ratio does not cause significant changes in $\lambda[7]$.

This model makes use of ADComputeDilatationThermalExpansionEigenstrainBase to compute the thermal strain tensor from the linear thermal expansion function.

\subsubsection{Creep}

ADMCCreepUpdate calculates the creep of mixed (U,Pu)C (monocarbides) due to applied stresses. The models employed are the thermal and irradiation creep rate formulations from [1]:

$$
\begin{aligned}
& \dot{\varepsilon}_{t o t a l}=\dot{\varepsilon}_{t h}+\dot{\varepsilon}_{i r r}, \\
& \dot{\varepsilon}_{t h}=1.49 \times 10^{10} \sigma^{2.44} \exp (-63200 / T), \\
& \dot{\varepsilon}_{i r r}=3.6 \times 10^{-22} \mathrm{F \sigma}
\end{aligned}
$$

where the total strain rate $\dot{\varepsilon}_{\text {total }}(1 / \mathrm{h})$ is split into the thermal and irradiation contributions, $\dot{\varepsilon}_{t h}$ and $\dot{\varepsilon}_{i r r}$ respectively. $T$ is temperature (K), $\sigma$ is the effective stress $(\mathrm{MPa})$, and $F$ is the fission rate $\left(\# / \mathrm{cm}^{3} / \mathrm{s}\right)$. The $\dot{\varepsilon}_{i r r}$ model roughly agrees with that suggested in [6], but the $\dot{\varepsilon}_{t h}$ model results in creep rates an order of magnitude lower than in [6].

\subsubsection{Swelling}

The ADMCVolumetricSwellingEigenstrain material model computes an eigenstrain tensor that accounts for solid and gaseous swelling and for densification in mixed ( $\mathrm{U}, \mathrm{Pu}) \mathrm{C}$ (monocarbides). This model uses correlations from [1] to compute the swelling increment for UC per percent of burnup:

$$
\begin{gathered}
\frac{\Delta V}{V}=0.4667+1.711 f_{\phi} \\
T<700^{\circ} \mathrm{C}
\end{gathered}
$$


and

$$
\begin{gathered}
\frac{\Delta V}{V}=0.4667+1.711 f_{\phi}+\left[\left(6.412-0.0198 T+0.152 \times 10^{-4} T^{2}\right) f_{B} f_{\phi}\right] \\
T>700^{\circ} \mathrm{C}
\end{gathered}
$$

where $T$ is temperature in Celsius, $\phi$ is the porosity, and $\Delta V / V$ is given per $10 \mathrm{MW}-\mathrm{d} / \mathrm{kg}$ (i.e., $1 \%$ burnup). The correction factors for porosity $f_{\phi}$ and for burnup $f_{B}$ are

$$
\begin{aligned}
f_{\phi}\left(\phi, p_{\text {contact }}\right) & =\exp (0.04-\phi) \exp \left[-\left(\frac{p_{\text {contact }}}{p_{0}} b\right)\right], \quad \phi \geq 0 \\
f_{B}(B) & =\frac{B}{B_{0}}-a, \quad f_{B} \geq 0
\end{aligned}
$$

where $p_{\text {contact }}$ is the contact pressure (MPa) and $B$ is the burnup (MW-d/kg). The suggested fit parameters are

$$
\begin{aligned}
a & =2 \mathrm{MW}-\mathrm{d} / \mathrm{kg} \\
b & =0.1 \\
p_{0} & =1 \mathrm{MPa} \\
B_{0} & =10 \mathrm{MW}-\mathrm{d} / \mathrm{kg}
\end{aligned}
$$

An upper limit on swelling of $\Delta V / V=4.558$ is suggested by [1].

The model also includes a densification term for $\left(\mathrm{U}_{0.85} \mathrm{Pu}_{0.15}\right) \mathrm{C}$ from [8]

$$
\Delta \phi=\Delta \phi_{\max }\left(1-e^{-B / B_{c}}\right)
$$

where $\Delta \phi_{\max }=-0.034$ is the maximum porosity change and $B_{c}=0.006$ FIMA. Volumetric densification is then computed by

$$
\frac{\Delta V}{V}=\frac{\Delta \phi}{1-\left(\phi_{0}+\Delta \phi\right)}
$$

where $\phi_{0}$ is the initial (as-fabricated) porosity.

\subsection{Mixed Nitrides}

\subsubsection{Thermal properties}

The material model ADMNThermal calculates the thermal conductivity $\lambda$ and specific heat capacity $c_{p}$ of UN.

The thermal conductivity $\lambda$ is given in $\mathrm{W} / \mathrm{m}-\mathrm{K}$ by [4] as a function of porosity $\phi$ :

$$
\begin{gathered}
\lambda=1.864 e^{-2.14 \phi} T^{0.361} \\
298<T<1923 \mathrm{~K} .
\end{gathered}
$$
[5]:

Specific heat capacity $c_{p}$ is calculated in $\mathrm{J} / \mathrm{mol}-\mathrm{K}$ as a function of temperature according to the correlation from

$$
\begin{gathered}
c_{p}=51.14\left(\frac{\Theta}{T}\right)^{2} \frac{\exp (\Theta / T)}{[\exp (\Theta / T)-1]^{2}}+9.491 \times 10^{-3} T+\frac{2.6415 \times 10^{11}}{T^{2}} \exp (-18081 / T) \\
298<T<2628 \mathrm{~K} .
\end{gathered}
$$

where $\Theta=365.7 \mathrm{~K}$ is the experimentally determined Einstein temperature of UN.

Note that the specific heat capacity given above is converted to $\mathrm{J} / \mathrm{kg}-\mathrm{K}$ by the code for use in BISON. 


\subsubsection{Elasticity}

The ADMNElasticityTensor material model computes the elasticity tensor for UN (mononitrides) using temperatureand porosity-dependent values for the Young's modulus and Poission's ratio as reported by [3]. The Young's modulus, $E$, is computed in units of MPa as:

$$
\begin{gathered}
E=0.258(100-\phi)^{3.002}\left(1-2.375 \times 10^{-5} T\right) \\
298<T<1473 \mathrm{~K} \\
0<\phi<30 \%
\end{gathered}
$$

where $T$ is the temperature in $\mathrm{K}$ and $\phi$ is the percent porosity.

For consistent property calculations, the correlation for Poisson's ratio $v$ must be computed from the same data as $E$, so we again use [3]:

$$
\begin{gathered}
v=1.26 \times 10^{-3}(100-\phi)^{1.174} \\
298<T<1473 \mathrm{~K} \\
0<\phi<30 \%
\end{gathered}
$$

where again, the porosity is in percent.

This material is assumed to be isotropic, and $E$ and $v$ are therefore used to generate an isotropic elasticity tensor. The values for $E$ and $v$ are given by [3] over the ranges of porosity and temperature shown above, so an exception is thrown if the porosity or temperatures are outside of this range.

\subsubsection{Creep}

ADMNCreepUpdate calculates the thermal and irradiation creep of UN (mononitride) due to applied stresses

$$
\dot{\varepsilon}_{t o t a l}=\dot{\varepsilon}_{t h}+\dot{\varepsilon}_{i r r}
$$

The thermal creep rate with porosity correction is from [3]. The creep rate $\dot{\varepsilon}_{T D}(1 / \mathrm{s})$ at theoretical density is

$$
\dot{\varepsilon}_{T D}=2.054 \times 10^{-3} \sigma^{4.5} \exp (-39369.5 / T) .
$$

$T$ is temperature (K) and $\sigma$ is the effective stress (MPa). A porosity correction must be applied according to

$$
f(\phi)=\frac{0.987}{(1-\phi)^{27.6}} \exp (-8.65 \phi)
$$

where $\phi$ is the porosity. The correction is multiplied, i.e. $\dot{\varepsilon}_{t h}=\dot{\varepsilon}_{T D} \times f(\phi)$.

The creep due to irradiation is from [9], based on a typical porosity of $4 \%$,

$$
\dot{\varepsilon}_{i r r}=2 \times 10^{-22} \sigma F
$$

where $\sigma$ is the effective stress (MPa), $F$ is the fission rate $\left(\# / \mathrm{cm}^{3} / \mathrm{s}\right)$, and the creep $\dot{\varepsilon}_{i r r}$ is given in $(1 / \mathrm{h})$.

\subsubsection{Thermal Expansion}

The ADMNThermalExpansionEigenstrain material model computes an eigenstrain tensor that accounts for thermal expansion in UN (uranium mononitride). This model uses the correlation of [2] to compute the coefficient of linear thermal expansion $\lambda$

$$
\begin{gathered}
\lambda=6.6774 \times 10^{-6}+1.4093 \times 10^{-9} T \\
298<T<2523 \mathrm{~K}
\end{gathered}
$$

where $T$ is temperature in $\mathrm{K}$ and $\lambda$ is given in $1 / \mathrm{K}$. The equation is independent of porosity because porosity does not have a significant influence on thermal expansion [6].

This model makes use of ADComputeDilatationThermalExpansionEigenstrainBase to compute the thermal strain tensor from the linear thermal expansion function. 
Table 5.1: WSA-32 Rod Design Parameters

\begin{tabular}{lccc} 
Parameter & Value & Units & Source \\
\hline Clad material & $316 \mathrm{SS}$ & & {$[11]$} \\
Clad bonding & $\mathrm{He}$ & & {$[11]$} \\
Clad OD & 9.40 & $\mathrm{~mm}$ & {$[11]$} \\
Clad thickness & 0.508 & $\mathrm{~mm}$ & {$[11]$} \\
Diametral gap & $0.15,0.28$ & $\mathrm{~mm}$ & {$[11]$} \\
Pellet diameter & $8.10,8.23$ & $\mathrm{~mm}$ & {$[11]$} \\
Fuel stack & 343 & $\mathrm{~mm}$ & {$[6]$} \\
Plenum height & 43.5 & $\mathrm{~mm}$ & {$[6]$, see discussion } \\
Plenum pressure & 12.4 & $\mathrm{MPa}$ & {$[6]$} \\
Top/bottom gap & 8 & $\mathrm{~mm}$ & unknown (estimated) \\
Smear density & $76,78,81$ & $\% \mathrm{TD}$ & {$[11]$}
\end{tabular}

\subsubsection{Swelling}

The ADMNVolumetricSwellingEigenstrain material model computes an eigenstrain tensor that accounts for volumetric swelling in UN (mononitride). This correlation was developed for space nuclear power systems by [10]. The model computes the swelling increment by percent volume $\Delta V / V(\%)$ :

$$
\frac{\Delta V}{V}=4.7 \times 10^{-11} T^{3.12} B^{0.83} \phi^{0.5},
$$

where $T$ is the temperature (K), $B$ is the burnup (at.\%), and $\phi$ is the as-fabricated fuel density (\% of theoretical).

\subsection{Fuel Rod Demonstrations}

\subsubsection{Carbides: EBR-II WSA-32 Experiment}

\subsubsection{Overview}

The WSA-32 experiment in EBR-II contained a series of mixed uranium-plutonium carbide fuel pins run to high burnup (12 at.\%) at high power $(100 \mathrm{~kW} / \mathrm{m})$ over the period from 1976-1981. The WSA-32 test contained 19 pins operated at the expected midplane peak power for a hypothetical 91-pin assembly in the Fast Flux Test Facility. Postirradiation analysis included profilometry, fission gas release, and ceramographic/microsturctural examination.

\subsubsection{Test Description}

The WSA-32 experiment consisted of 19 helium-bonded pins, five of which were replaced with fresh pins after a burnup of $8.6 \%$. The design parameters that were varied were the pellet densities and the diametral gap sizes. The irradiation reached peak burnup of $12 \%$.

The fuel was solid cylindrical pellets of $\left(\mathrm{U}_{0.8} \mathrm{Pu}_{0.2}\right) \mathrm{C}$ with sesquicarbide content of $10 \pm 5$ volume percent and $79.3 \%$ enriched uranium content. The pellet densities were 81 and $87 \%$ TD and the diametral gap sizes were 0.15 and $0.28 \mathrm{~mm}$, resulting in smear densities of 76, 78, and $81 \%$ (87\% TD pellets were not used in the smaller gap configuration). The pellets were encased in $316 \mathrm{SS}$ clad and wire-wrapped with a $1.7 \mathrm{~mm}$ diameter spacer, also in 316 SS.

The plenum volume according to [6] is $2.4 \mathrm{~cm}^{3}$, which results in a plenum height of just $43.5 \mathrm{~mm}$. However, this number needs to be confirmed since it is inconsistent with other experiments in EBR-II, which typically had much longer fuel pins.

The actual power history for specific EBR-II experiments is still being determined. Therefore, a simplified power history containing an initial ramp to power and hold for a given amount of time with a final power down is being used. The average burnup of the fuel at the end of the simulation is used as a check that the power history is reasonable. The remaining parameters are in 5.2. 
Table 5.2: WSA-32 Operating Parameters

\begin{tabular}{lccc} 
Parameter & Value & Units & Source \\
\hline Coolant $T_{\text {in }}$ & 644 & $\mathrm{~K}$ & {$[6]$} \\
Coolant $T_{\text {out }}$ & 746 & $\mathrm{~K}$ & {$[6]$} \\
Average flux & $1.6 \times 10^{15}$ & $\mathrm{n} \mathrm{cm}^{-2} \mathrm{~s}^{-1}$ & {$[6]$} \\
Peak power & $100-107$ & $\mathrm{~kW} / \mathrm{m}$ & {$[6],[11]$} \\
Burnup & $4-12$ & at. $\%$ & {$[11]$}
\end{tabular}

Table 5.3: WS32-02 Peaking Factors [11]

\begin{tabular}{lcc} 
Axial position (x/L) & Linear power $(\mathrm{kW} / \mathrm{m})$ & Burnup (at.\%) \\
\hline 0.11 & 86.4 & 10.0 \\
0.30 & 96.6 & 10.9 \\
0.56 & 99.7 & 11.3 \\
0.70 & 95.9 & 10.9 \\
0.89 & 86.8 & 9.8
\end{tabular}

\subsubsection{BISON Model}

The test case simulates the W32-02 rod, which had a diametral gap of $0.29 \mathrm{~mm}$ and initial density of $88.1 \%$ TD according to profilimetry data, resulting in a smear density of $82.2 \%$. We simulated this rod because it has relatively low porosity, and some of the models in BISON have a limit of $15 \%$ porosity. The axial peaking factors and burnups for W32-02 are shown in 5.3.

The 2D-RZ mesh for the test case is generated with the internal smeared pellet meshing capability in BISON [SmearedPelletMeshGenerator](/SmearedPelletMeshGenerator.md). All of the dimensions and meshing details are contained in the Mesh block.

This test case uses automatic differentiation. The following material and behavioral models for the (U,Pu)C fuel were used:

- ADMCElasticityTensor

- ADMCCreepUpdate

- ADMCThermal

- ADMCThermalExpansionEigenstrain

- ADMCVolumetricSwellingEigenstrain

The following material and behavioral models for the 316SS cladding were used in these cases:

- ADComputeIsotropicElasticityTensor

- ADSS316Thermal

- ADDensity

This case demonstrates use of automatic differentiation with full (SMP) preconditioning. Therefore it is missing models for mechanical and thermal contact, which are not implemented yet. To simulate mechanical contact, a constant plenum pressure is applied to all pellet boundaries.

To simulate thermal contact, the steady-state radial heat flux is computed from the fission rate, and this flux is used to compute the clad and gap temperature drops. Since the Na coolant temperature is prescribed, the temperature at the pellet OD can then also be prescribed as a function of local coolant temperature and fission rate. This method avoids the ill-posed BC that would result from assigning a heat flux directly to pellet boundary. 
Table 5.4: K-4 Rod Design Parameters

\begin{tabular}{lccc} 
Parameter & Value & Units & Source \\
\hline Clad material & $316 \mathrm{SS}$ & & {$[12]$} \\
Clad bonding & $\mathrm{He}$ & & {$[12]$} \\
Clad OD & 7.87 & $\mathrm{~mm}$ & {$[12]$} \\
Clad thickness & 0.51 & $\mathrm{~mm}$ & {$[6]$} \\
Diametral gap & 0.65 & $\mathrm{~mm}$ & calculated from smear density \\
Pellet diameter & 6.20 & $\mathrm{~mm}$ & calculated from smear density \\
Fuel stack & 343 & $\mathrm{~mm}$ & unknown, using WSA32 \\
Plenum height & 43.5 & $\mathrm{~mm}$ & unknown, using WSA32 \\
Plenum pressure & 12.4 & $\mathrm{MPa}$ & unknown, using WSA32 \\
Top/bottom gap & 8 & $\mathrm{~mm}$ & unknown, using WSA32 \\
Smear density & $81.2,79.4$ & $\% \mathrm{TD}$ & {$[12]$}
\end{tabular}

Table 5.5: K-4 Operating Parameters

\begin{tabular}{lccc} 
Parameter & Value & Units & Source \\
\hline Coolant $T_{\text {in }}$ & 644 & $\mathrm{~K}$ & unknown, using WSA32 \\
Coolant $T_{\text {out }}$ & 746 & $\mathrm{~K}$ & unknown, using WSA32 \\
Fast fluence & $6 \times 10^{22}$ & $\mathrm{n} \mathrm{cm}^{-2}$ & {$[12]$} \\
Peak power & 85 & $\mathrm{~kW} / \mathrm{m}$ & {$[12]$} \\
Burnup & 9.6 & at. $\%$ & {$[12]$}
\end{tabular}

\subsubsection{Nitrides: EBR-II K-4 Experiment}

\subsubsection{Overview}

The K-4 experiment in EBR-II contained a series of mixed uranium-plutonium nitride fuel pins run to high burnup $(9.6$ at. \%) at high power $(85 \mathrm{~kW} / \mathrm{m})$. The K-4 test is considered the most successful UN test, achieving high burnup with no pin failures. Unfortunately, although the K-4 pins were examined in detail, the results of the examination were not published [12].

\subsubsection{Test Description}

The K-4 experiment consisted of over $140(\mathrm{U}, \mathrm{Pu}) \mathrm{N} \mathrm{Na}$ - and helium-bonded pins that were irradiated as part of the LMFBR program with low smear densities of $75-86 \%$ TD to ensure little to no fuel-clad mechanical interaction.

The fuel was solid cylindrical pellets of (U,Pu)N. The pellet densities were 96.8 and $98.9 \% \mathrm{TD}$, with smear densities of 81.2 and $79.4 \%$, respectively. The pellets were encased in 316 SS clad and 316 SS shroud and wirewrapped, also in $316 \mathrm{SS}$. We do not model the shroud tubes or the wire wrap.

The plenum volume and some other properties are unknown, so we are using the configuration from the WSA32 test (see other assessment case) at EBR-II to fill in the details.

The actual power history for specific EBR-II experiments is still being determined. Therefore, a simplified power history containing an initial ramp to power and hold for a given amount of time with a final power down is being used. The average burnup of the fuel at the end of the simulation is used as a check that the power history is reasonable. The remaining parameters are in 5.5 .

\subsubsection{BISON Model}

The test case simulates a rod in the set of eight pins which had a fuel density of $96.8 \%$ TD and a smear density of $79.4 \%$. Using these densities with a clad thickness of $0.51 \mathrm{~mm}$ gives a pellet diameter of $6.20 \mathrm{~mm}$ and a diametral gap of $0.65 \mathrm{~mm}$. No data on axial peaking factors exist, so we again use the factors from the WSA32 test. 
The 2D-RZ mesh for the test case is generated with the internal smeared pellet meshing capability in BISON [SmearedPelletMeshGenerator](/SmearedPelletMeshGenerator.md). All of the dimensions and meshing details are contained in the Mesh block.

This test case uses automatic differentiation. The following material and behavioral models for the (U,Pu)N fuel were used:

- ADMNElasticityTensor

- ADMNCreepUpdate

- ADMNThermal

- ADMNThermalExpansionEigenstrain

- ADMNVolumetricSwellingEigenstrain

The following material and behavioral models for the 316SS cladding were used in these cases:

- ADComputeIsotropicElasticityTensor

- ADSS316Thermal

- ADDensity

This case demonstrates use of automatic differentiation with full (SMP) preconditioning. Therefore it is missing models for mechanical and thermal contact, which are not implemented yet. To simulate mechanical contact, a constant plenum pressure is applied to all pellet boundaries.

To simulate thermal contact, the steady-state radial heat flux is computed from the fission rate, and this flux is used to compute the clad and gap temperature drops. Since the Na coolant temperature is prescribed, the temperature at the pellet OD can then also be prescribed as a function of local coolant temperature and fission rate. This method avoids the ill-posed BC that would result from assigning a heat flux directly to pellet boundary. 


\section{Bibliography}

[1] Timm Preusser. Modeling of carbide fuel rods. Nuclear Technology, 57(3):343-371, 1982.

[2] S.L. Hayes, J.K. Thomas, and K.L. Peddicord. Material property correlations for uranium mononitride: I. physical properties. Journal of Nuclear Materials, 171(2):262 - 270, 1990.

[3] S.L. Hayes, J.K. Thomas, and K.L. Peddicord. Material property correlations for uranium mononitride: Ii. mechanical properties. Journal of Nuclear Materials, 171(2):271 - 288, 1990.

[4] S.L. Hayes, J.K. Thomas, and K.L. Peddicord. Material property correlations for uranium mononitride: Iii. transport properties. Journal of Nuclear Materials, 171(2):289 - 299, 1990.

[5] S.L. Hayes, J.K. Thomas, and K.L. Peddicord. Material property correlations for uranium mononitride: Iv. thermodynamic properties. Journal of Nuclear Materials, 171(2):300 - 318, 1990.

[6] Hubert Blank. Nonoxide Ceramic Nuclear Fuels. John Wiley \& Sons, Ltd, 2006.

[7] Hj Matzke. Science of advanced LMFBR fuels. Elsevier Science Pub. Co. Inc, 1986.

[8] W. Dienst. Swelling, densification and creep of (u, pu)c fuel under irradiation. Journal of Nuclear Materials, 124:153 - 158, 1984.

[9] D. Brucklacher and W. Dienst. Creep behavior of ceramic nuclear fuels under neutron irradiation. Journal of Nuclear Materials, 42(3):285-296, 1972.

[10] Steven B. Ross, Mohamed S. El-Genk, and R.Bruce Matthews. Uranium nitride fuel swelling correlation. Journal of Nuclear Materials, 170(2):169 - 177, 1990.

[11] P.J. Levine, U.P. Nayak, and A. Boltax. High burnup, high power irradiation behavior of helium-bonded mixed carbide fuel pins. In Trans. 7th Int. Conf. on SMiRT, volume C5/2, pages 161-168, Chicago, 1981. NorthHolland; Amsterdam.

[12] R B Matthews. Irradiation performance of nitride fuels. Technical report, Los Alamos National Lab, 11993. 


\section{Chapter 6}

\section{ACTIVITIES}

The activities for FY-20 are listed in Table 6.1.

Table 6.1: FY 2020 Activities for NEAMS INL-engineering scale fuel performance effort

\begin{tabular}{lc}
\hline Milestone & Completion Date \\
\hline $\begin{array}{l}\text { Provide user training and support for simulating metallic, parti- } \\
\text { cle, and UN/UC fuels }\end{array}$ & $9 / 30$ \\
\hline $\begin{array}{l}\text { Particle Fuel Model Assessment_IAEA Benchmarks and AGR } \\
\text { Validation }\end{array}$ & $9 / 30$ \\
\hline
\end{tabular}

\subsection{User Training and Support}

Several BISON user training sessions were performed both on-site and virtually during the course of FY 2020. User support was provided to multiple organizations including the NRC, ORNL and a number of commercial entities regarding metallic and particle fuel modeling.

\subsection{Particle Fuel Model Assessment}

The assessment of the particle fuel modeling capability currently in BISON progressed steadily over the course of FY 2020. A comparison between BISON and PARFUME for representative benchmark problems was completed. In addition, the AGR-5/6/7 experiments were simulated using BISON and the results have been presented in this report. Futher details can be found in chapter 4. 


\section{Chapter 7}

\section{FUTURE WORK}

This section summarizes the proposed future work in the previous sections and attempts to prioritize these suggested research activities. Since this summary report is essentially organized in sections related to different fuel types (i.e., metallic, TRISO, and UN/UC), this same structure will be used here.

Firstly, although metallic fuels have been used in many reactors (EBR-II, FFTF, etc...) and a large database of experimental data exists, a number of critical behaviors in $\mathrm{U}-\mathrm{Pu}-\mathrm{Zr}$ type fuels is not well understood. These include $\mathrm{Zr}$ redistribution, swelling, fission gas release, and fuel-cladding interactions. Since much of the experimental data and experience in metallic fuel is from the 1960s through the early 1990s, new experiments both in and out of reactor will be needed to validate the existing models for $\mathrm{U}-\mathrm{Pu}-\mathrm{Zr}$ fuel performance. Unfortunately, the defunding of the metallic fuel related aspects of the AFC program makes it unlikely that will occur.

Therefore, a continuing priority in metallic fuel research should be the usage of existing experimental data and establishing the best model parameters through calibration of the current models. This approach can be applied to each of the research areas mentioned above with the priorities being:

1. Swelling (particularly at low burnups and anisotropic effects)

2. Zr Redistribution

3. Fuel-Cladding Interactions

\section{Fission Gas Release}

Secondly, the TRISO fuel type is being used in several advanced reactor designs and appears to be much closer to new commercial use than the other fuels. Therefore, an increased focus on the modeling needs related to regulatory and safety issues is essential. Simulating important failure mechanisms and comparing those results to existing experimental data should be a high priority for FY-21. Incorporating the latest material property experimental results into BISON and improving fission product release predictions for various fuel designs using TRISO particles is a crucial step in the commercialization process.

Lastly, even though the UN/UC fuel types are still in the relatively early phase of commercial development, modeling efforts need to be maintained in order to support the advanced reactor designs based on these fuels. The addition of more material models into BISON for the relevant phenomenon in UN/UC such as fission gas release and the effects of irradiation on the thermal and mechanical properties is vital. In addition, BISON simulations of UN/UC fuel pins and comparisons to the available experimental data will provide validation of the code and highlight future modeling needs. 


\section{Chapter 8}

\section{ACKNOWLEDGEMENTS}

The submitted manuscript has been authored by a contractor of the U.S. Government under Contract DE-AC0705ID14517. Accordingly, the U.S. Government retains a non-exclusive, royalty free license to publish or reproduce the published form of this contribution, or allow others to do so, for U.S. Government purposes. 